\title{
A Pathway for the Development of Advanced Reactor Mechanistic Source Term Modeling and Simulation Capabilities
}

Nuclear Science and Engineering Division, Argonne National Laboratory 


\section{About Argonne National Laboratory}

Argonne is a U.S. Department of Energy laboratory managed by UChicago Argonne, LLC under contract DE-AC02-06CH11357. The Laboratory's main facility is outside Chicago, at 9700 South Cass Avenue, Argonne, Illinois 60439. For information about Argonne and its pioneering science and technology programs, see www.anl.gov.

\section{DOCUMENT AVAILABILITY}

Online Access: U.S. Department of Energy (DOE) reports produced after 1991 and a growing number of pre-1991 documents are available free at OSTI.GOV (http://www.osti.gov/), a service of the US Dept. of Energy's Office of Scientific and Technical Information.

\section{Reports not in digital format may be purchased by the public from the} National Technical Information Service (NTIS):

U.S. Department of Commerce

National Technical Information Service

5301 Shawnee Rd

Alexandria, VA 22312

www.ntis.gov

Phone: (800) 553-NTIS (6847) or (703) 605-6000

Fax: (703) 605-6900

Email: orders@ntis.gov

Reports not in digital format are available to DOE and DOE contractors from the Office of Scientific and Technical Information (OSTI):

U.S. Department of Energy

Office of Scientific and Technical Information

P.O. Box 62

Oak Ridge, TN 37831-0062

www.osti.gov

Phone: (865) 576-8401

Fax: (865) 576-5728

Email: reports@osti.gov

\section{Disclaimer}

This report was prepared as an account of work sponsored by an agency of the United States Government. Neither the United States Government nor any agency thereof, nor UChicago Argonne, LLC, nor any of their employees or officers, makes any warranty, express or implied, or assumes any legal liability or responsibility for the accuracy, completeness, or usefulness of any information, apparatus, product, or process disclosed, or represents that its use would not infringe privately owned rights. Reference herein to any specific commercial product, process, or service by trade name, trademark, manufacturer, or otherwise, does not necessarily constitute or imply its endorsement, recommendation, or favoring by the United States Government or any agency thereof. The views and opinions of document authors expressed herein do not necessarily state or reflect those of the United States Government or any agency thereof, Argonne National Laboratory, or UChicago Argonne, LLC. 


\section{A Pathway for the Development of Advanced Reactor Mechanistic Source Term Modeling and Simulation Capabilities}

Prepared by

Shayan Shahbazi and David Grabaskas

Nuclear Science and Engineering Division, Argonne National Laboratory

May 2021 


\section{Acknowledgements}

This report is part of a larger collaboration between Argonne National Laboratory and Sandia National Laboratories to assist in the development of modern mechanistic source term modeling and simulation tools for advanced nuclear reactors, as part of the U.S. Department of Energy (DOE) Nuclear Energy Advanced Modeling and Simulation (NEAMS) program. The authors would like to thank Bo Feng, Tanju Sofu, and Christopher Stanek for their support in this effort.

The authors would like to thank the following for providing valuable input or review of this work: Jennifer Leute, Kenneth Wagner, David Luxat, Andrew Clark, Sara Thomas, Fred Gelbard, Jake McMurray, Ted Besmann, Markus Piro, Ling Zou, Stephen Novascone, Paolo Balestra, and David Andersson.

The input of the advanced reactor industry was vital in the development of the current work. The authors would like to thank the following vendors who participated in the development of this work or preceding efforts:

Framatome

$\mathrm{X}$-Energy

Flibe Energy

Kairos Power

Seaborg Technologies

Copenhagen Atomics

Elysium Industries

ThorCon

Terrestrial Energy

Moltex Energy

TerraPower

GE-Hitachi Nuclear Energy

Advanced Reactor Concepts

Oklo

Westinghouse 


\section{Executive Summary}

Source term analysis is a vital aspect of advanced reactor (non-LWR) design and licensing activities. The pursuit of mechanistic source term (MST) analyses, utilizing realistic models for radionuclide transport and retention phenomena for specific scenarios, offers improved insights into reactor behavior that can support risk-informed design and regulatory decision-making. To further the development of MST modeling and simulation (mod/sim) capabilities for the advanced reactor community, the U.S. Department of Energy (DOE) Nuclear Energy Advanced Modeling and Simulation (NEAMS) program established an MST research collaboration between Argonne National Laboratory (Argonne) and Sandia National Laboratories (SNL).

Building on the initial FY20 efforts, the current work seeks to create an MST research and development pathway for advanced reactor MST mod/sim capabilities. The pathway establishes long-term development objectives in pursuit of creating comprehensive MST mod/sim tools, while also identifying critical near-term tasks. The objectives of the pathway include supporting the U.S. Nuclear Regulatory Commission (NRC) with its MST code development activities as part of the DOE/NRC memorandum of understanding, in addition to supporting the needs of the advanced reactor industry in the performance of MST analyses for design and licensing calculations.

For this work, Argonne interacted extensively with vendors to ensure that the views of the advanced reactor industry were properly captured within the developed pathway. SNL assisted in the coordination with the NRC code development plan, including providing content specific to the MELCOR and MACCS codes.

The following document outlines the long-term development objectives and critical near-term tasks for each advanced reactor type, along with consequence modeling (i.e., offsite dispersion and dose assessment). The activities have been evaluated based on need and projected effort for completion. In addition, interfaces and cross-cutting activities with other DOE program areas have been identified. Coordination between these technical areas is vital for overall future project success. The activities identified within the pathway will be utilized by the NEAMS MST research project to guide and prioritize subsequent efforts. 


\section{Table of Contents}

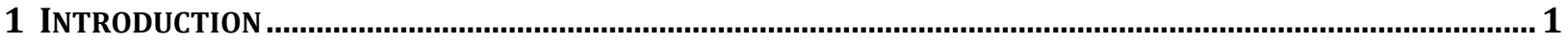

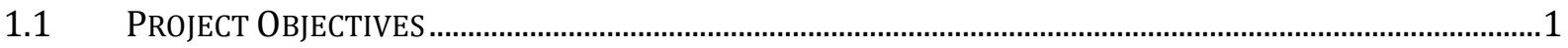

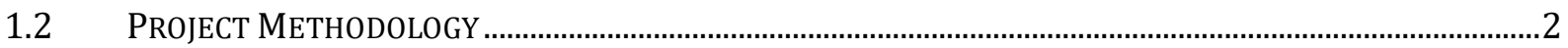

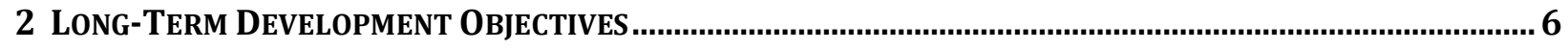

2.1 HTGR

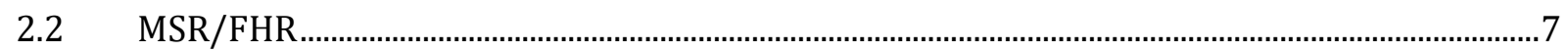

2.3 SFR.

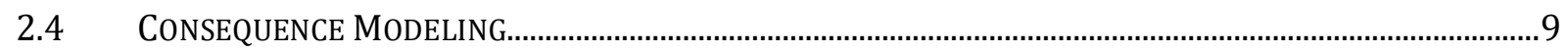

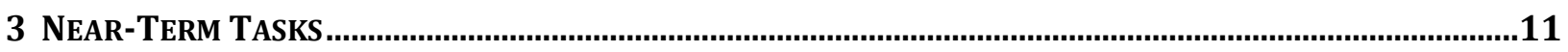

3.1 HTGR

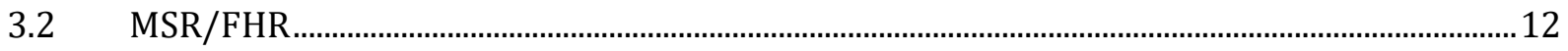

3.3 SFR

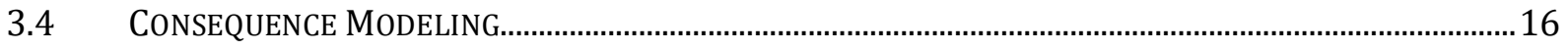

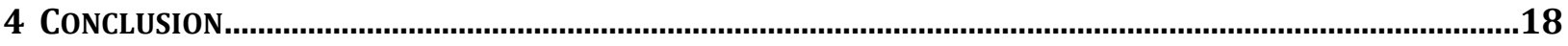

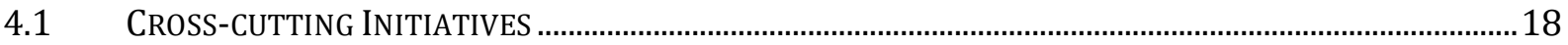

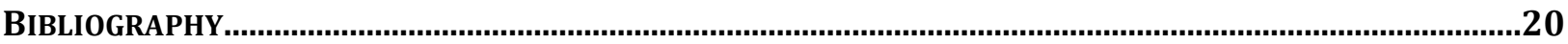

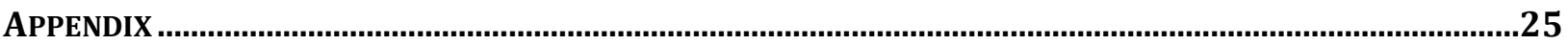

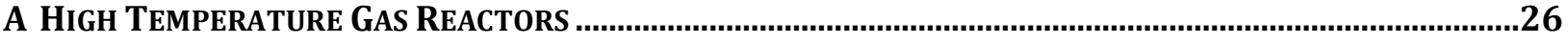

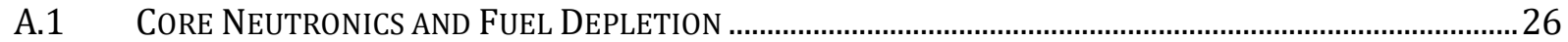

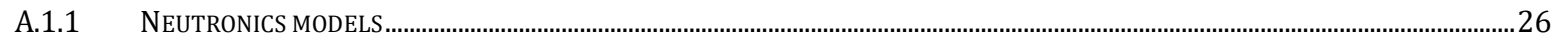

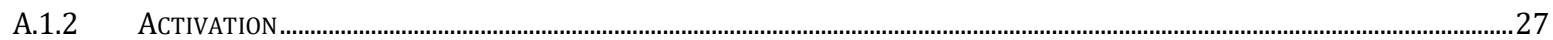

A.2 TRISO FUEL PARTICLE RELEASES..................................................................................................... 27

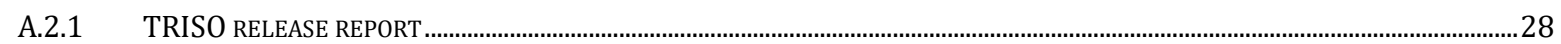

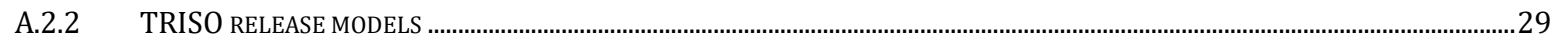

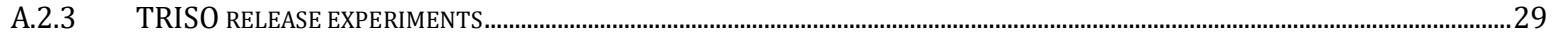

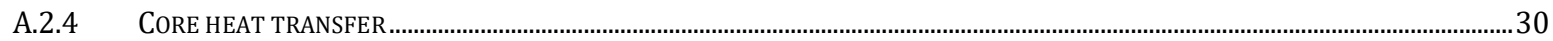

A.3 RADIONUCLIDE TRANSPORT IN CORE MATERIALS ..................................................................................... 31

A.3.1 RADIONUCLIDE TRANSPORT AND OXIDATION BEHAVIOR REPORT ……................................................................................

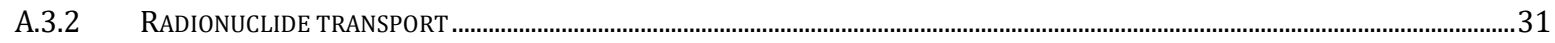

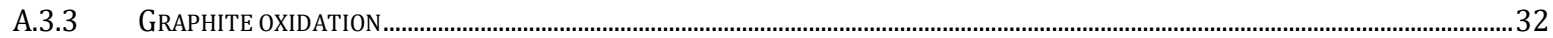

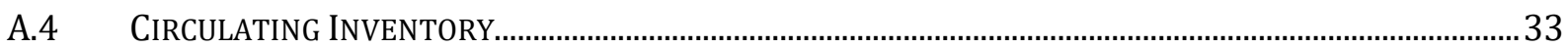

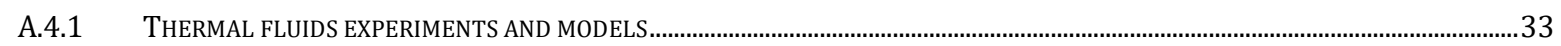

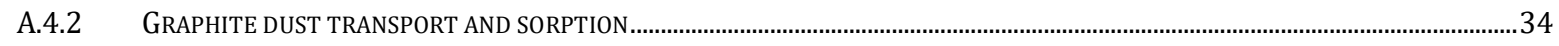

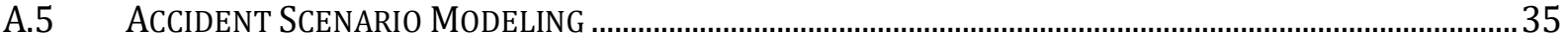

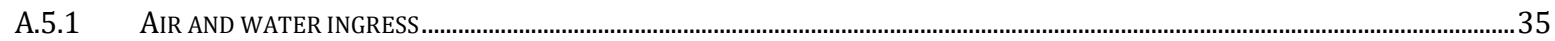

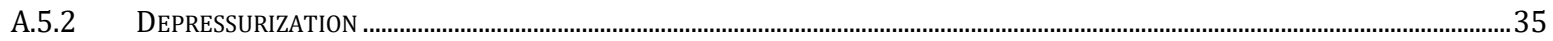

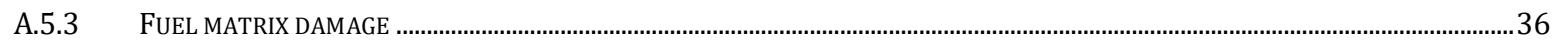

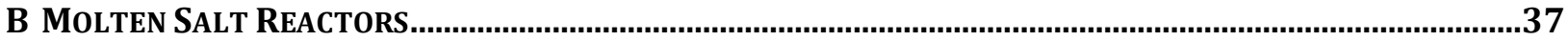

B.1 CORE NEUTRONICS AND FUEL DEPLETION (SALT-FUELED MSR ONLY)................................................ 37

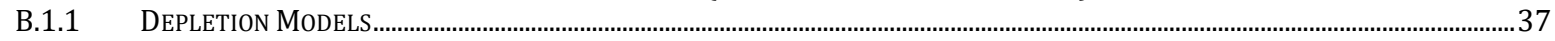

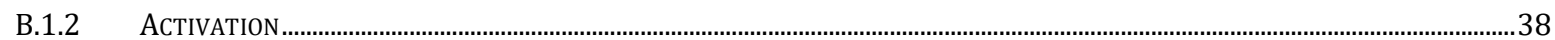

B.2 CORE NEUTRONICS AND FUEL DEPLETION (FHR ONLY) .........................................................................39

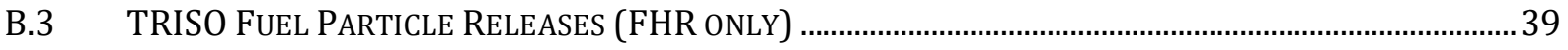

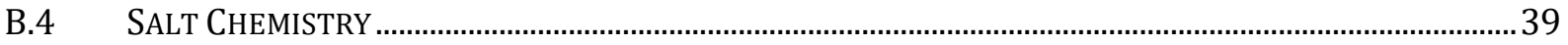

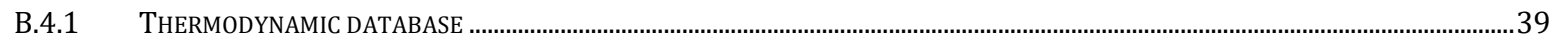

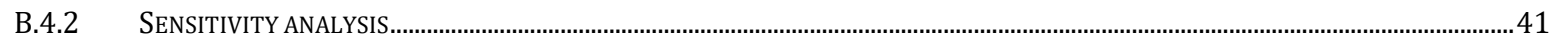

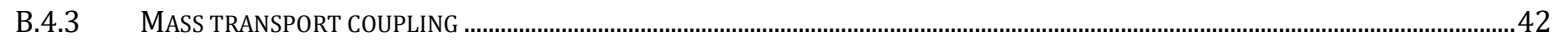

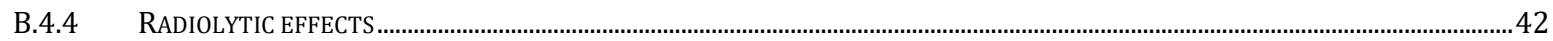

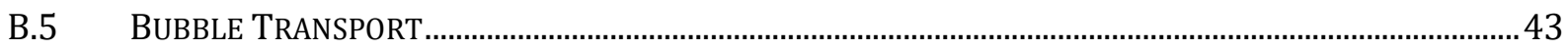




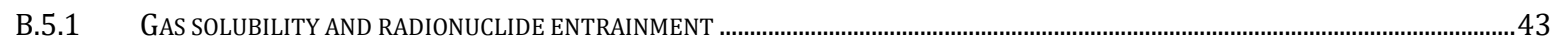

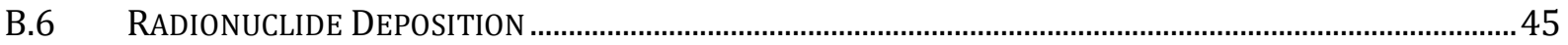

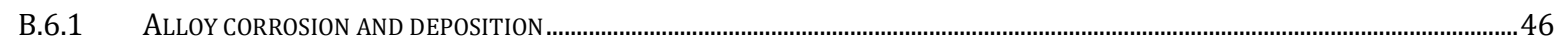

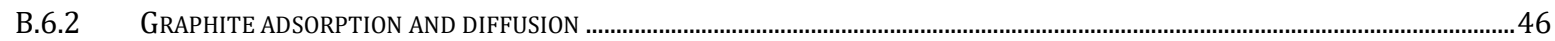

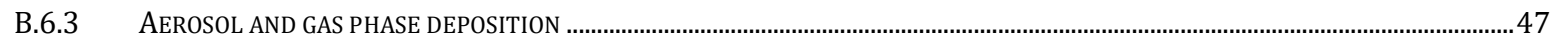

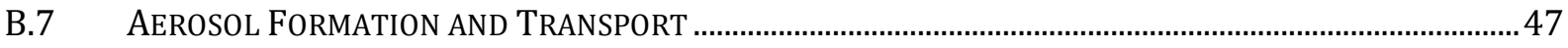

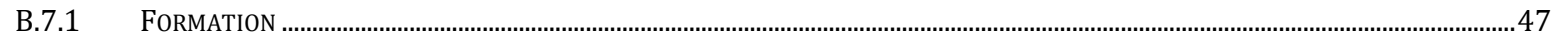

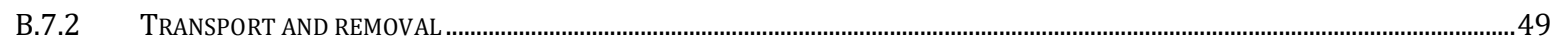

B.7.3 APPLICABILITY OF EXISTING AEROSOL MODELS....................................................................................................................

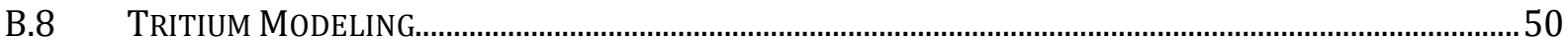

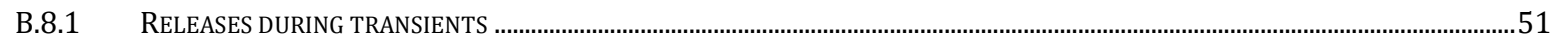

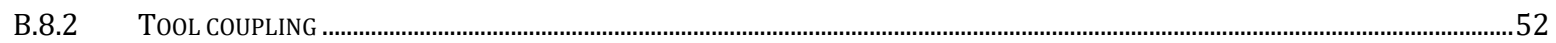

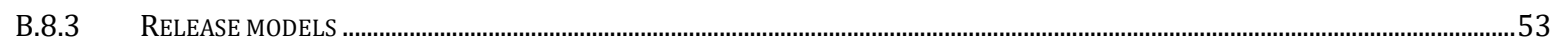

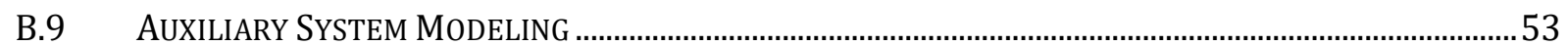

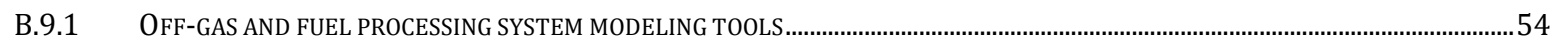

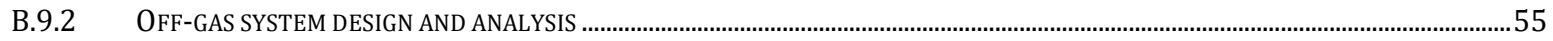

B.9.3 NOBLE METAL BEHAVIOR IN OFF-GAS SYSTEM …..............................................................................................................5

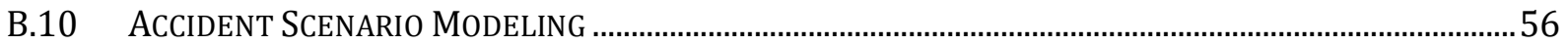

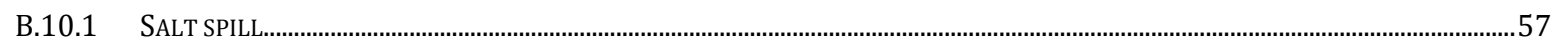

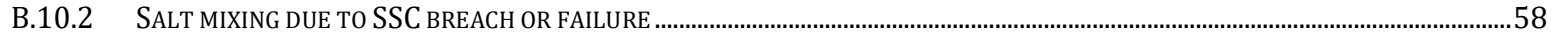

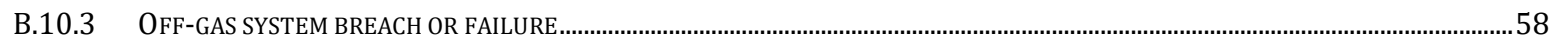

B.10.4 DAMAGE TO FUEL MATRIX (FHR ONLY)

C SODIUM-COOLED FAST REACTORS .....................................................................................................60

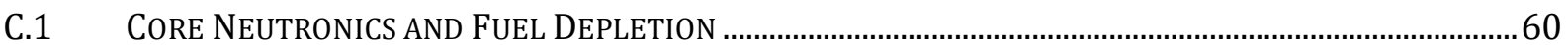

C.2 RADIONUCLIDE IN-PIN MIGRATION AND RELEASE .................................................................................6 60

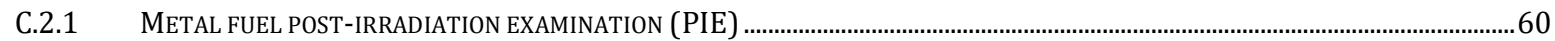

C.2.2 RADIONUCLIDE MIGRATION MODELING DURING PRE-TRANSIENT IRRADIATION .........................................................................61

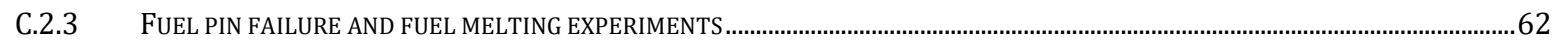

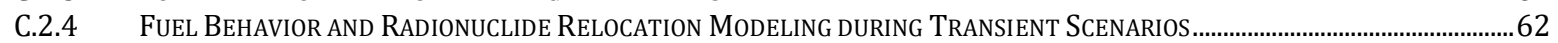

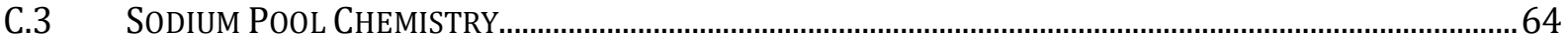

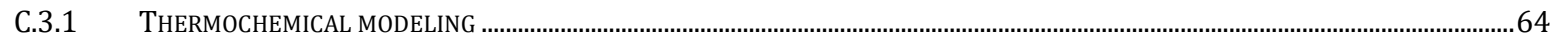

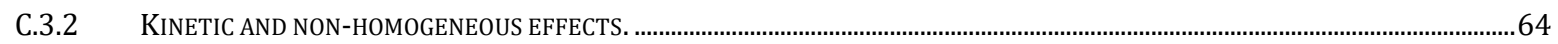

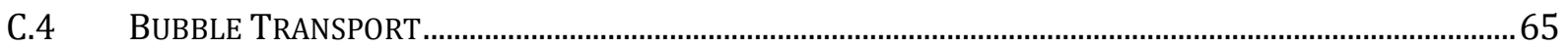

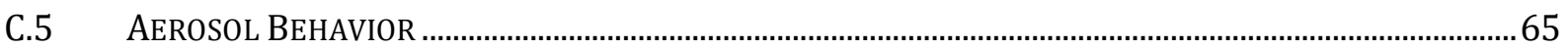

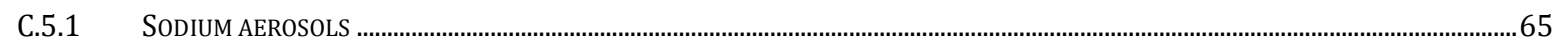

C.5.2 LEAKAGE AND PATHWAY PLUGGING BEHAVIOR

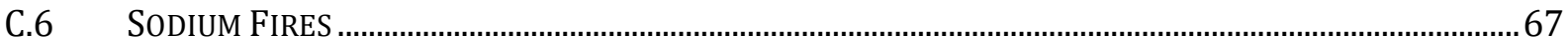

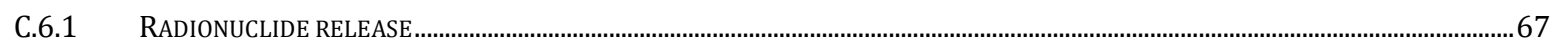

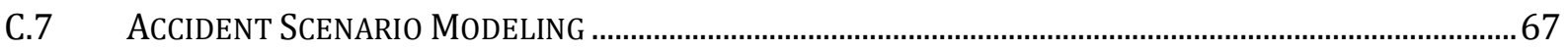

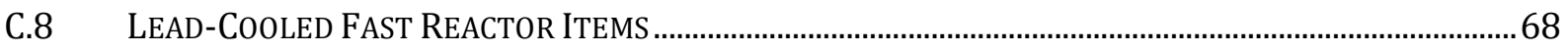

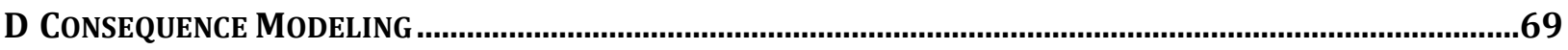

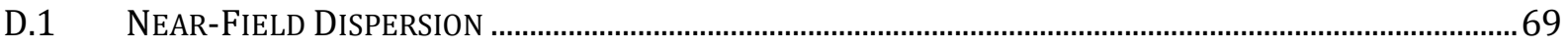

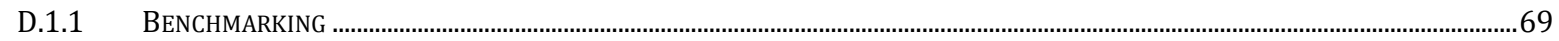

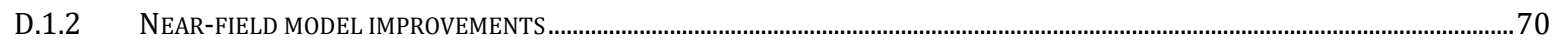

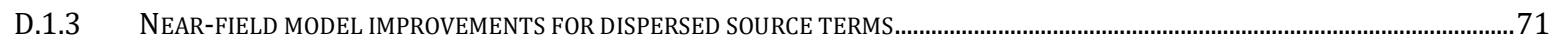

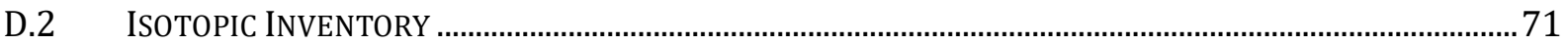

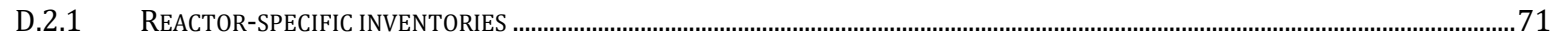

D.2.2 REACTOR-SPECIFIC RADIONUCLIDES AND CHEMICAL FORMS RELEASED ……..............................................................................72

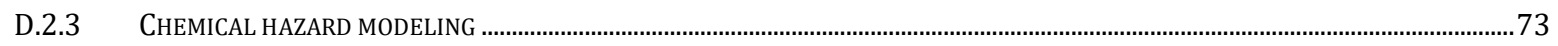

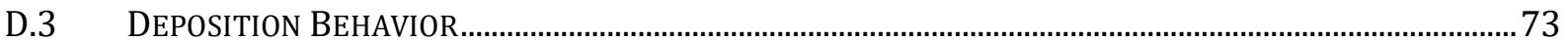

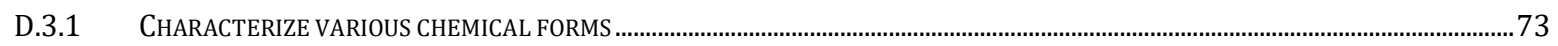

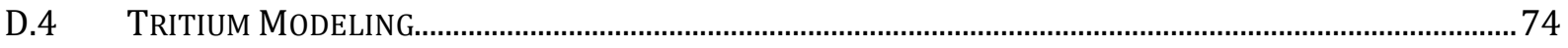

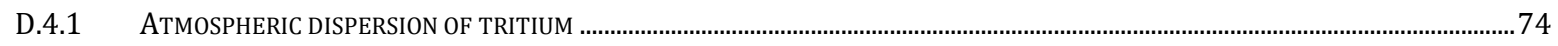

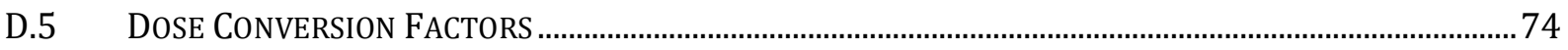

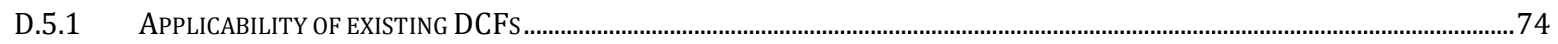

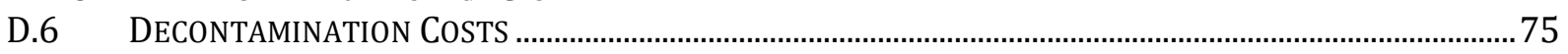

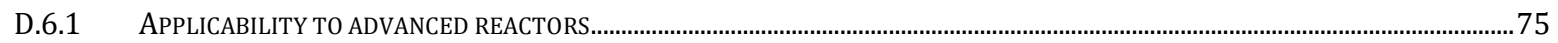




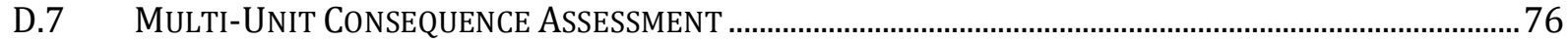

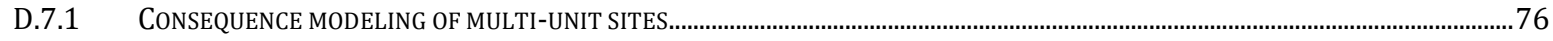




\section{Acronyms}

\begin{tabular}{|c|c|}
\hline Acronym & Definition \\
\hline AERMOD & American Meteorological Society / Environmental Protection Agency Regulatory Model \\
\hline AFC & Advanced Fuels Campaign \\
\hline ARCON96 & Atmospheric Relative Concentrations in Building Wakes \\
\hline ART & DOE's Advanced Reactor Technology research program \\
\hline AVR & Arbeitsgemeinschaft Versuchsreaktor (German pebble bed reactor) \\
\hline CFD & computational fluid dynamics \\
\hline CNWG & Civil Nuclear Energy Research and Development Working Group \\
\hline DCF & dose conversion factors \\
\hline DOE-NE & U.S. Department of Energy, Office of Nuclear Energy \\
\hline EBR-II & Experimental Breeder Reactor II \\
\hline EPZ & emergency planning zones \\
\hline FAI & Fauske \& Associates, Inc \\
\hline FATE & Facility flow, Aerosol, Thermal, and Explosion code \\
\hline FFTF & Fast Flux Test Facility \\
\hline FHR & fluoride salt-cooled high temperature reactor \\
\hline GEM & Gibbs Energy Minimization solver \\
\hline HSC & chemistry modeling software \\
\hline HTGR & high temperature gas-cooled reactor \\
\hline HTR & See HTGR \\
\hline HYSPLIT & HYbrid Single-Particle Lagrangian Integrated Trajectory \\
\hline IFR & Integral Fast Reactor \\
\hline INL & Idaho National Laboratory \\
\hline LBE & licensing basis events \\
\hline LFR & lead-cooled fast reactor \\
\hline LMP & Licensing Modernization Project \\
\hline LWR & light-water reactor \\
\hline MACCS & MELCOR accident consequence code system \\
\hline MOU & memorandum of understanding \\
\hline MSM & Molten Salt Model (within MELCOR) \\
\hline MSR & molten salt reactor \\
\hline MSRE & Molten Salt Reactor Experiment \\
\hline MST & mechanistic source term analysis \\
\hline MSTDB-TC & Molten Salt Thermodynamic Database, Thermochemical Properties \\
\hline NEAMS & Nuclear Energy Advanced Modeling and Simulation program \\
\hline NEUP & Nuclear Energy University Program \\
\hline NGNP & Next Generation Nuclear Plant \\
\hline NRC & Nuclear Regulatory Commission \\
\hline ORIGEN & ORNL Isotope Generation and Depletion Code \\
\hline ORNL & Oak Ridge National Laboratory \\
\hline
\end{tabular}


PAVAN Program for the Meteorological Evaluation of Non-Routine Releases from Nuclear Power Stations

PBMR pebble bed modular reactor

PIE $\quad$ post-irradiation examination

PIRT phenomena identification and ranking table

QA quality assurance

QUIC Quick Urban and Industrial Complex

SAM System Analysis Module

SCALE Standardized Computer Analyses for Licensing Evaluation code suite

SFR sodium-cooled fast reactor

SHARC Specialized Hazard Assessment Response Capability

SNL Sandia National Laboratories

SRT Simplified Radionuclide Transport code

SSC structures, systems, and components

TRISO tri-structural isotropic fuel

UCO uranium oxycarbide

VTR Versatile Test Reactor 


\section{Introduction}

Source term analysis, or the estimation of the potential radionuclide release to the environment during reactor events, is a central focus of the reactor licensing process and a vital part of riskinformed reactor design approaches. A mechanistic source term (MST) analysis is designed to realistically model the release and transport of radionuclides from the source to the environment for specific scenarios, while accounting for retention or transmutation phenomena and associated uncertainties. The objective of MST analyses, in comparison to bounding or conservative source term assessments, is to provide a non-biased representation of reactor risk and improve the information available for siting, emergency planning, and reactor design decisions. In support of the advanced reactor community in its pursuit of MST analysis capabilities, this work outlines a recommended research pathway for the development of MST modeling and simulation (mod/sim) tools.

\subsection{Project Objectives}

To assist both the advanced reactor industry and U.S. Nuclear Regulatory Commission (NRC) in the pursuit of reactor design and licensing, the U.S. Department of Energy (DOE) Nuclear Energy Advanced Modeling and Simulation (NEAMS) program has established an MST research project under the auspices of the Application Drivers technical area. The MST research project is a collaboration between Argonne National Laboratory (Argonne) and Sandia National Laboratories (SNL) with a high-level objective to coordinate the development of comprehensive advanced reactor MST mod/sim capabilities to support risk-informed design and licensing decisions. However, this goal can be further discretized into several supporting objectives, as outlined in Figure 1.

Main Objective: Coordinate the development of comprehensive advanced reactor MST analysis capabilities to support risk-informed design and licensing decisions.
NRC
Support the NRC's
development of selected
tools for advanced reactor
MST licensing analyses,
such as MELCOR and
MACCS, as part of the
DOE-NRC MOU

Industry

Support the advanced

reactor industry and their

ability to conduct MST

analyses for design

decisions and licensing submittals

\section{NEAMS}

To the extent practical,

leverage and expand the

MST modeling capabilities

and insights of NEAMS

$\mathrm{mod} / \mathrm{sim}$ tools

Figure 1: Project Objectives

First, as detailed in the recently signed Addendum No. 3 to the DOE-NRC Memorandum of Understanding (MOU) [1], the project seeks to support the NRC's development of its selected advanced reactor licensing MST mod/sim tools, such as MELCOR and MACCS. Since the NRC has already outlined their code development plans for MST analysis tools in ref [2], as part of their non-LWR vision and strategy, the current project seeks to perform complementary activities that further advance this approach. Second, the project seeks to support the advanced reactor 
industry and their ability to perform MST analyses for design decisions and licensing submittals. This may include supporting their use of NRC tools or providing data and insights in support of other modeling pathways. In addition, the project seeks to leverage and expand the MST capabilities and insights of existing NEAMS mod/sim tools.

Given the extensive ongoing activities within NEAMS and other programs regarding advanced reactor multi-physics and thermal fluids modeling, the MST research project is primarily focused on the modeling of radionuclide release and transport rather than reactor behavior during transients. However, given the inherent dependencies of radionuclide transport behavior on reactor conditions, this linkage is an important aspect that requires close coordination between the MST research project and other programs and areas (e.g., through benchmarking activities).

\subsection{Project Methodology}

To achieve the objectives described in the preceding subsection, the project team has utilized a structured approach for identifying and understanding the pertinent transient scenarios for each advanced reactor type, the phenomena important to advanced reactor MST development, and the tools and methods available to model these phenomena. Initial efforts, completed in FY20, focused on these aspects of the project and are outlined in Figure 2.

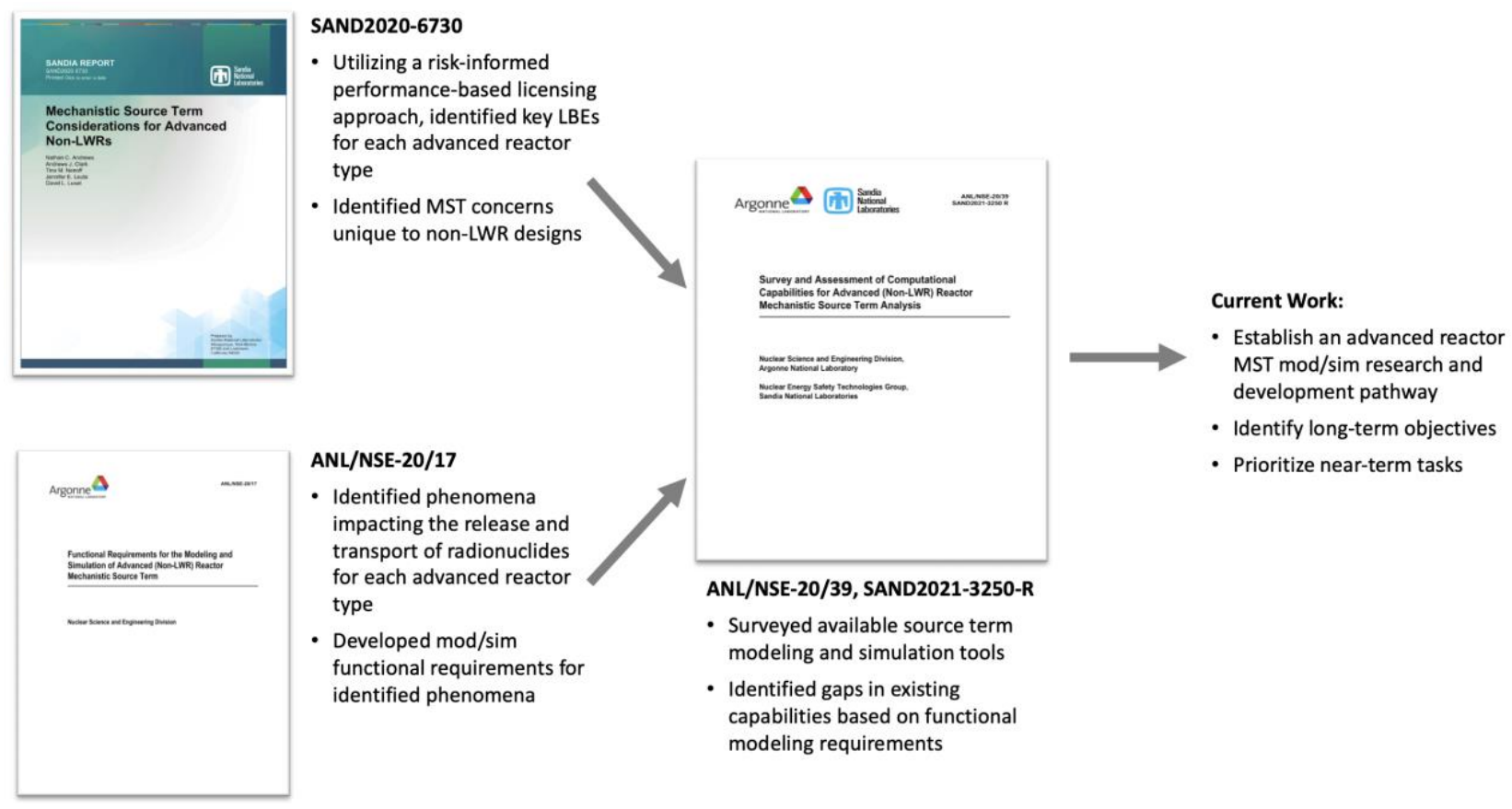

Figure 2: Overview of Project Efforts

Ref [3] identified the probable licensing basis events (LBEs) for each advanced reactor type, in addition to MST modeling concerns unique to non-LWR designs. In parallel, ref [4] developed comprehensive functional requirements for MST modeling based on identified phenomena related to radionuclide release and transport for each advanced reactor type. Using this information, ref [5] performed a survey and assessment of available MST mod/sim tools with the goal of identifying gaps in their current capabilities. 
Utilizing the findings of the previous efforts, the current work aims to establish an MST research and development pathway to resolve key gaps in the mod/sim of advanced reactor MST. The developed pathway has two primary objectives:

- Outline Long-Term Development Objectives: Provide multi-year objectives and guidance for the development of comprehensive advanced reactor MST mod/sim capabilities.

- Prioritize Near-Term Tasks: Identify and prioritize those tasks that must be completed in the near-term to address critical industry needs or that are important predecessors to long-term development objectives.

The recommended actions resulting from this assessment include both the development of new $\mathrm{mod} / \mathrm{sim}$ capabilities but also improving the confidence in existing tools through benchmarking and validation exercises, as outlined in Figure 3.

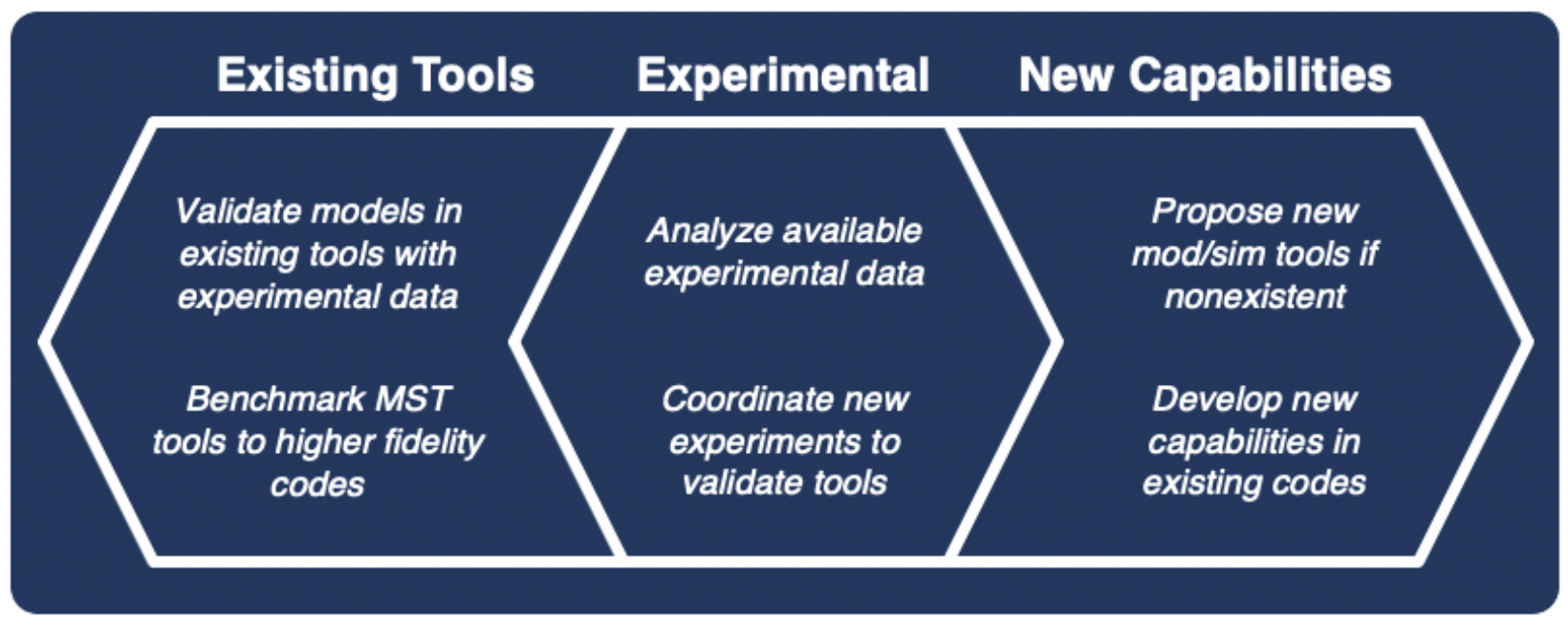

Figure 3: General Types of Gap Resolution Actions

To create the MST mod/sim development pathway, the project utilized the following series of steps:

- Gap Identification: Identification of mod/sim gaps in available MST tools, completed as part of ref [5].

- Gap Resolution Action Creation: Coordination with the NRC computer code development plan to identify tasks that are complementary to those being pursued by the NRC. Discussions with the advanced reactor industry to identify avenues to address key needs.

- Gap Resolution Priority and Timeline: Discussions with the advanced reactor industry to determine the prioritization of gaps and necessary timeline of gap resolution.

- Gap Resolution Effort: Interfaces with other DOE-NE areas and programs to refine the resolution actions and establish estimates regarding the effort associated with each action. 
To properly capture the needs and recommendations of the advanced reactor industry, the Argonne project team met with the vendors outlined in Table 1. This includes both U.S. advanced reactor vendors and several international vendors who are potentially interested in the U.S. as a deployment location. In general, the project focused on the non-microreactor advanced reactor industry, as given the size of microreactors, there may be preference to utilize bounding source term assessments. ${ }^{1}$ Within the current work, the insights from industry are not attributed to specific vendors but are generalized to protect any potential proprietary information.

Table 1: Advanced Reactor Industry Contributors

\begin{tabular}{|c|c|c|}
\hline HTGR & MSR/FHR & SFR/LFR ${ }^{1}$ \\
\hline $\begin{array}{l}\text { - Framatome } \\
\text { - X-Energy }\end{array}$ & $\begin{array}{l}\text { - Flibe Energy } \\
\text { - Seaborg Technologies } \\
\text { - Copenhagen Atomics } \\
\text { - Elysium Industries } \\
\text { - ThorCon } \\
\text { - Terrestrial Energy } \\
\text { - Moltex Energy } \\
\text { - TerraPower } \\
\text { - Kairos Power }\end{array}$ & $\begin{array}{l}\text { - TerraPower } \\
\text { - GE-Hitachi Nuclear Energy } \\
\text { - Advanced Reactor Concepts } \\
\text { - Oklo } \\
\text { - Westinghouse }\end{array}$ \\
\hline
\end{tabular}

${ }^{1}$ Includes those vendors who participated in the development of ref [6], which was used as guidance for the current work.

As a result of the process outlined above, a series of gap resolution actions were identified and assessed for each advanced reactor type as well as consequence modeling. The gap resolutions are described in detail within a series of appendices, as outlined in Figure 4. The appendices contain a review of the identified gap resolution actions for each advanced reactor type and consequence modeling, including justification for the priority level, effort level, and timeline for each action, along with cross-cutting activities under the NRC's code development pathway [2]. Portions of the appendix were provided by SNL and are extracted from ref [7].

The main body of the report summarizes the information contained within the appendices, with Section 2 providing an overview of the long-term project objectives for each advanced reactor type. Section 3 highlights the identified near-term tasks that will be prioritized by the research program. This is followed with a conclusion in Section 4, which summarizes the project findings and highlights several key cross-cutting interfaces with other DOE-NE programs and the NRC.

\footnotetext{
${ }^{1}$ There may be benefits to the use of an MST assessment even for microreactors or other facility with a comparatively small radionuclide inventory, such as the justification of very small sites or reduced emergency planning requirements. In general, the capabilities to be developed as part of the outlined MST research pathway may also be applicable to advanced microreactor designs.
} 


\section{Section 1: Introduction}

Section 2: Long-Term Development Objectives

Section 3: Near-Term Tasks

Section 4: Conclusion and Crosscutting Interfaces

Appendix: Detailed Gap Resolution Actions

A: High Temperature Gas Reactors

B: Molten Salt Reactors

C: Sodium Fast Reactors

D: Consequence Modeling

Figure 4: Report Outline 


\section{Long-Term Development Objectives}

The detailed resolution pathway assessment described within the appendices, in conjunction with the high-level project objectives described in Section 1.1, informed the selection of reactorspecific long-term development objectives, which are summarized in the following subsections. The long-term development objectives provide multi-year direction to the project in its efforts to meet the ultimate goal of developing comprehensive advanced reactor MST mod/sim capabilities.

\subsection{HTGR}

To improve confidence in MST modeling for HTGRs, development in several key technical areas is recommended. These long-term development objectives are briefly discussed here and are in accordance with the important phenomena, relevant computational capabilities, and the resulting information gaps which have been outlined in previous reports, as well as a previous HTGR phenomena identification and ranking table (PIRT) exercise completed as part of the NGNP project for the NRC, which considered fission product transport and dose [4, 5, 8]. The long-term development objectives are summarized below and are described in more detail in Appendix A:

\section{- Core Neutronics and Fuel Depletion}

○ Develop models for various generic core geometries for HTGR concepts in NRCsupported code suites such as SCALE

○ Develop models for the activation of coolant or core materials, such as potential impurities of lithium in graphite, reflectors, or core materials, as well as helium

- TRISO Fuel Particle Releases

O Develop models within source term/severe accident modeling codes on radionuclide release fractions from TRISO fuel that are dependent on fuel type, failure mode, radionuclide class, and temperature, in addition to baseline releases from manufacturing defects and transient failures

○ For model validation, complete TRISO irradiation experiments to confirm release and failure behavior and validate the models

- Radionuclide Transport in Core Materials

- Develop models for the transport of radionuclides through the fuel matrix and core materials, including potential radionuclide release during graphite oxidation

- For model validation, perform experimental studies on radionuclide diffusion transport, adsorption into carbon, carbide formation, and graphite oxidation leading to heat generation and the release of radionuclides and combustible gases

- Circulating Inventory

○ Develop models for the deposition, liftoff, and wash-off of condensable radionuclides in the primary circuit at representative system pressures and temperatures; Validate these models with the experimental studies

- Continue developing thermal fluids models for the transport and deposition of radionuclides through the primary circuit, as well as those modeling the liftoff and wash-off during accident scenarios such as depressurizations; Validate these models with the experimental studies

- Develop models and design validation experiments aimed at elucidating the formation mechanism and quantities formed of graphite dust in HTGRs as a function of fuel matrix, core design, irradiation behavior, or other factors 
- Perform validation experiments on the sorption behavior of radionuclides (which may plate out or circulate in the primary circuit) onto graphite dust in the core and primary circuit, e.g., pressurized inert gas environments

- Accident Scenario Modeling

○ Individually investigate accident scenarios and sequence progression as the understanding of the above phenomenology improves and as reactor design details become available

Given the importance of the TRISO fuel particle on radionuclide retention in HTGR systems, the outlined objectives focus foremost on accurately modeling the behavior and potential release of radionuclides from TRISO fuel particles and other core materials, such as graphite matrices. Additional objectives center on the development of modeling capabilities for circulating inventory within the primary system, such as dust or released radionuclides, including during the conditions associated with system depressurization or graphite oxidation events. This objective also connects with the goal to properly understand the potential activation of other materials within the system, including those with the potential to be available for release within the primary circuit.

\section{$2.2 \mathrm{MSR} / \mathrm{FHR}$}

The long-term objectives for MSR and FHR MST mod/sim are briefly described here and are in accordance with the important phenomena, relevant computational capabilities, and the resulting information gaps which have been outlined in previous reports $[4,5]$ and expand on recent efforts in MSR MST model development $[9,10]$. The long-term development objectives are summarized below and are described in more detail in Appendix B:

\section{- Core Neutronics and Fuel Depletion}

○ Develop generalized modeling tools for MSR and FHR fuel depletion as well as transmutation in fuel or coolant salts to improve confidence in radionuclide inventory calculations, noting potential impurities and corrosion products

- For FHRs: Analyze existing data and develop models for radionuclide releases from TRISO fuel particles as a function of time, temperature, chemical group, and other characteristics of the particle such as defects (See Appendix A.2)

\section{- Salt Chemistry}

○ Continue development of molten salt thermodynamic database and solver which improves confidence in chemical and phase equilibrium calculations for radionuclide speciation and phase changes in molten salt systems

- Develop radionuclide species-tracking tool which couples aforementioned thermodynamic modeling tool with various relevant mass transport phenomena, including those listed in the following sections

- Develop and validate models on radiolytic effects in molten salts and its impact on radionuclide transport during normal operation as well as accident scenarios

\section{- Bubble Transport}

○ In support of model development and validation, complete studies on bubble transport in molten salts, investigating volatile fission product solubility, radionuclide entrainment in flowing bubbles, noble metal behavior as surfactants, and the impact of bubble bursting at surfaces on aerosol formation 


\section{- Radionuclide Deposition}

- Develop models related to the various modes of radionuclide deposition from fluids onto various material surfaces such as alloys or graphite

- Develop models on radionuclide diffusion into and release from graphite as a function of temperature

- Aerosol Formation and Transport

O Develop models for molten salt aerosol formation, transport, and removal mechanisms and complete ongoing salt spill experimental studies for validation.

\section{- Tritium Modeling}

○ If deemed necessary due to radiological impact, implement tritium-specific transport models within severe accident analysis or system-level analysis codes to aid in the simulation of tritium releases during accident or transient scenarios

- Complete experimental studies to better understand tritium releases from graphite or other trapping materials during temperature transients

- Auxiliary System Modeling

○ Develop modeling tools for the auxiliary systems proposed alongside the primary loop reactor system as details become available

$\circ$ Integrate chemistry knowledge specific to the auxiliary systems into the modeling tools

- Coordinate experiments aimed at investigating noble metal-specific behavior in off-gas systems such as gas phase transport and deposition

\section{- Accident Scenario Modeling}

○ Individually investigate accident scenarios and sequence progression as the understanding of the above phenomenology improves and as reactor design details become available

Because MSRs are strongly multiphysics in nature, many of the above development objectives are cross-cutting with other MSR-related research programs. Computational challenges in reactor physics for MSRs, which are being addressed, will also provide for improvements in radionuclide inventories. Salt chemistry plays a pivotal role in the design, operation, safety, and decommissioning of MSRs, and therefore this development objective overlaps with ongoing research which supports all these facets. Solubility, precipitation, vaporization, bubble transport, and aerosol formation all affect reactivity of the nuclear reactor in some way, and thus this supports other research considering operational concerns. Additionally, off-gas system modeling research, as well as that for other auxiliary systems such as on-line fuel processing, affects the operation, safety, and material accountancy of MSRs. And finally, accident scenario modeling will also motivate design choices while potentially optimizing the economics of MSRs. Even though the source term during some accident scenarios may be largely contained, the exercise may still provide insight on reactor optimizations such as material choices.

\subsection{SFR}

In general contrast to other advanced reactor types, significant work has been performed in support of SFR MST analysis, due in large part to both historical and recent DOE efforts [6, 11, 12]. The results of these projects provide vital insights to remaining gap areas. Based on this information and the assessments performed as part of the current project, the long-term objectives for SFR MST development include the following: 
- Radionuclide Migration and Release from Failed Fuel Pins

$\circ$ Further development and validation of mechanistic models for in-pin radionuclide migration during irradiation

- Further development and validation of mechanistic models for radionuclide release during fuel pin failure

- Radionuclide Behavior in the Sodium Pool

- Validation of existing mechanistic models for radionuclide bubble transport in liquid sodium

○ Validation and potential improvement of mechanistic models for the vaporization of radionuclides from the sodium pool

- Radionuclide Aerosol and Vapor Behavior

○ Validation and potential improvements to existing mechanistic models for vapor and aerosol behavior within the cover gas region and containment/confinement

- Development of mechanistic models for assessment of vapor and aerosol leakage pathway plugging

- Accident Scenario Modeling

○ Increase the understanding of metal fuel behavior under certain transient conditions, in addition to characterizing potential accidents associated with sodium clean-up systems

Due to past model development and data collection efforts, the long-term objectives for SFR MST focus on completing or enhancing the development and validation of mechanistic models for multiple phenomena. These tasks include detailed modeling of radionuclide migration and release from metal fuel pins within the core and improvements to existing modeling approaches for assessing radionuclide transport within the sodium pool. Lastly, the project also seeks to improve the understanding and modeling of sodium aerosols and vapors within the cover gas region and containment/confinement, including the possibility of leakage pathway plugging due to sodium oxide creation.

Although not explicitly outlined here, the SFR long-term objectives also generally apply to LFR designs. While there are differences in the proposed fuel forms, the models associated with radionuclide transport within the coolant pool and reactor system will likely be applicable to both SFR and LFR designs, provided that adequate lead-based data is available. The project plans to include LFR activities, to the extent possible, under the SFR area.

\subsection{Consequence Modeling}

Long-term objectives for consequence analysis modeling focus on improving the fidelity of current capabilities developed for LWRs, and tailoring tools to be more refined for advanced reactor applications. The long-term objectives for consequence analysis model development include the following:

\section{- Isotopic Inventory}

- Determining whether chemical hazards (such as beryllium and chlorine gas) represent a significant enough health hazard to be included in consequence analysis modeling alongside radioactive hazards. 
- Developing modeling capabilities to incorporate chemical hazards if necessary.

- Deposition Behavior

- Evaluating radionuclides with unique deposition behavior and creation of specific transport models, if necessary, e.g., tritium.

\section{- Dose Conversion Factors}

- Evaluating changes in radionuclides released, associated chemical forms, and particle size distributions to determine if changes are needed in dose conversion factors

- Evaluating changes in radionuclides released and associated chemical forms to determine if changes are needed in decontamination methods and cost modeling

- Near-Field Dispersion

- Modifications to Lagrangian particle tracking model integrated with MACCS to provide a higher fidelity near-field modeling capability compared to the Gaussian plume segment model.

- Multi-Unit Consequence Assessment

- Enhanced capability to model multiple source terms arising from spatially offset locations, and perform consequence analysis on multiple source terms in a simplified manner

- Modifying the underlying spatial setup to accommodate multiple offset source terms in a single consequence analysis.

○ Developing simplification methods to reduce the computational expense of multiple site and source term consequence analysis studies. 


\section{Near-Term Tasks}

The gap resolution assessment detailed within the appendices identified a series of near-term tasks that are vital to the completion of the long-term objectives described in the preceding section. The reactor-specific near-term tasks are summarized here, along with those pertaining to offsite consequence and dispersion modeling. These actions represent those items that the project seeks to potentially complete (whether through the project directly or the coordination with other programs) by the end of FY22, within the constraints of available resources.

\subsection{HTGR}

TRISO Fuel Particle Releases: HTGR vendors requested a summary of currently available data. This near-term task would catalog TRISO fission product release and failure data from past experimental tests. As documented and available, an assessment would be made on the quality assurance practices applied during to the experimental research. The report would specifically highlight modern UCO TRISO data versus older uranium-oxide fueled TRISO tests. The report would summarize the findings, overlapping measurements, and gaps. The task would produce a report summarizing the literature review of available TRISO fission product release data and an identification of gaps.

\begin{tabular}{|l|c|c|c|c|}
\hline Gap Resolution Action & Priority & Effort & Timeline & $\begin{array}{c}\text { NRC Vol3 } \\
\text { Crosscut }\end{array}$ \\
\hline $\begin{array}{l}\text { Summarize existing data on radionuclide releases } \\
\text { from TRISO fuel particles }\end{array}$ & High & Low & Near-term & HTGR-M2.1 \\
\hline
\end{tabular}

Radionuclide Transport in Core Materials: This near-term task would summarize the current understanding of radionuclide absorption into graphite, diffusion from the fuel compacts, and release during into the primary system during normal operation and during graphite oxidation accidents. The review will identify gaps and research needs, especially for UCO TRISO.

\begin{tabular}{|l|c|c|c|c|}
\hline Gap Resolution Action & Priority & Effort & Timeline & $\begin{array}{c}\text { NRC Vol3 } \\
\text { Crosscut }\end{array}$ \\
\hline $\begin{array}{l}\text { Summarize existing data on radionuclide } \\
\text { absorption into graphite, radionuclide carbide } \\
\text { formation, and graphite oxidation }\end{array}$ & Med & Low & Near-term & HTGR-M2.1 \\
\hline
\end{tabular}

Circulating Inventory: This near-term task would conduct modeling and experimentation related to the generation, transport, deposition, and liftoff of graphite dust, as well as the sorption of radionuclides to dust. The current understanding of modern graphite behavior following irradiation is a particular focus.

\begin{tabular}{|l|c|c|c|c|}
\hline Gap Resolution Action & Priority & Effort & Timeline & $\begin{array}{c}\text { NRC Vol3 } \\
\text { Crosscut }\end{array}$ \\
\hline $\begin{array}{l}\text { Conduct modeling and experimentation which } \\
\text { elucidate graphite dust formation, transport, and } \\
\text { radionuclide sorption phenomenology }\end{array}$ & Med & Med & Near-term & HTGR-M2.1 \\
\hline
\end{tabular}




\section{$3.2 M S R / F H R$}

The MSR/FHR near-term tasks are summarized below. Many are recommended starting points for resolving information gaps in the near-term, but most of them will have longer development timelines to be able to achieve complete resolution. Additionally, many of these technical area gap resolutions are cross-cutting in nature. Given the complexity of nuclear systems as a whole, especially those with a high degree of multiphysics phenomena such as MSRs, it will be especially important to utilize resources across multiple programs to develop these technical areas and close information gaps which affect different figures-of-merit of the reactor. For example, a recommended task to resolve source term modeling needs may also resolve information gaps related to corrosion modeling, instrumentation and control design, or some other operational characteristic important to the deployment of these reactors.

Salt Chemistry: As a result of discussions with vendors, the modeling phenomenon that was deemed to be the highest priority for resolution was information regarding salt chemistry. Specifically, reactor designers and analysts have many questions surrounding the vaporization of radionuclides from a molten salt solution, both inside the reactor vessel during normal operation, and outside the vessel in the case of a salt spill. Therefore, the first near-term high priority task is the continued development of the thermochemical properties of salt-soluble elements relevant to the source term and eventual inclusion into the Molten Salt Thermodynamic Database (MSTDBTC) [13-15]. This includes development of the Gibbs free energy minimization (GEM) solvers (e.g., Yellowjacket $[15,16]$ or Thermochimica [17-19]) which takes MSTDB data as input and estimates speciation and phase equilibria of the system based on thermodynamic equilibrium. In support of NEAMS, this development is already underway as it is a highly cross-cutting research initiative, but further emphasis is recommended on investigating the salt chemistry of all relevant fission products. This includes collecting experimental thermal analysis data of the fission product dissolved in various proposed salt systems to investigate the impact of non-ideal salt solution effects on the retention or vaporization of the fission product, which the theory of which was recently summarized with respect to several relevant fission products in a FLiBe salt system [20]. This tool will also be needed to model vaporization of radionuclides from a pool of molten salt formed after a spill, which has been identified as a potential accident scenario [21]. This could include vaporization as a function of temperature as well as volatilization of certain compounds as a result of radiolytic fluorine production as the irradiated salt cools [22].

A second near-term high priority task related to salt chemistry is the completion of a sensitivity study related to thermochemical modeling of radionuclides in molten salts. Simple thermodynamic equilibrium calculations rely on minimization of the Gibbs free energy of the components of the solution, but more robust solution models have been developed to account for the highly non-ideal behavior of molten salts when the solute is dissolved in the solution. This can have considerable impact on the source term as "negative" deviations from ideality will result in greater than expected retention in the salt, and "positive" deviations will result in greater instability in the salt solution [20]. The parameters of such a model are often not well defined and it is recommended that a sensitivity study be completed to assess the level of importance each parameter has on the results of the calculation given the unique system description of MSRs. Some examples of things that can be assessed for their impact are variations in the composition, redox potential differences, certain component thermodynamic data values, and the effect of temperature and pressure. The uncertainties associated with the underlying thermodynamic data 
should also be explored and propagated. The figures-of-merit worth investigating are the effect on vaporization, precipitation, and solubility.

\begin{tabular}{|l|l|l|l|c|}
\hline Gap Resolution Action & Priority & Effort & Timeline & $\begin{array}{c}\text { NRC Vol3 } \\
\text { Crosscut }\end{array}$ \\
\hline $\begin{array}{l}\text { Develop a thermodynamic database and GEM } \\
\text { solver of the necessary thermochemical properties } \\
\text { for molten fluoride and chloride salt systems, } \\
\text { including relevant base salt components but also } \\
\text { including fission products that may exist as soluble } \\
\text { in the salt solution; Validate the database }\end{array}$ & High & Med & Near-term & MSR-M3.2 \\
\hline $\begin{array}{l}\text { Complete a sensitivity analysis to investigate how } \\
\text { varying component thermodynamic values alters } \\
\text { computed chemical and phase equilibria, in } \\
\text { addition to an uncertainty propagation analysis }\end{array}$ & High & Low & Near-term & \\
\hline
\end{tabular}

Bubble Transport: Also related to salt chemistry, a near-term high priority task is recommended for the resolution of bubble transport modeling in molten salts, specifically investigating the solubility of various gaseous species, and the entrainment of radionuclides as bubbles transport through the salt. This resolution requires investigation into multiple mechanisms, including how radionuclides are entrained in sparged helium bubbles, how salt-borne noble gas fission products transport after being created, how noble metals may act as surfactants and transport on and influence the formation and mobility of bubbles, and also how bubble bursting at the surface may result in radionuclide vaporization or release, including the potential formation of aerosols. This is rated as high effort due to having a strong experimental component in closing these information gaps, as it will need to be proven if such models for aqueous systems are analogous.

\begin{tabular}{|l|c|c|c|c|}
\hline Gap Resolution Action & Priority & Effort & Timeline & $\begin{array}{c}\text { NRC Vol3 } \\
\text { Crosscut }\end{array}$ \\
\hline $\begin{array}{l}\text { Conduct experimental studies on the solubility, } \\
\text { bubble agglomeration, and transport of noble gases } \\
\text { and volatile fluorides through molten salts, as well } \\
\text { as corresponding radionuclide entrainment; } \\
\text { Develop and validate models }\end{array}$ & High & High & Near-term & MSR-M3.2 \\
\hline
\end{tabular}

Aerosol Formation and Transport: Aerosol formation and transport is another source term focus area of high importance, representing a common radionuclide transport mechanism for most nuclear reactor types. Unfortunately, the current state of knowledge surrounding molten salt aerosols is incomplete compared to aqueous aerosols. Resolving information gaps in aerosol formation mechanisms as well as aerosol transport and removal phenomena represent the next two near-term high priority tasks which are recommended here. A third task related to aerosol modeling which is recommended consists of analyzing the applicability of existing aerosol modeling knowledge (i.e., that developed for aqueous aerosols) to that for molten salts. As experiments in molten salt aerosols are completed, data should be analyzed to see if aqueous aerosol models can be confidently used to model salt aerosols. One example is improving confidence in the particle size distribution of salt aerosols as this affects transport, and thus ingestion. Experimental aerosol studies usually demand a high degree of effort, and this is even more true for aerosols formed at high temperatures, especially if the gas space should also be 
maintained at temperatures above ambient, which many regions of a nuclear system can be expected to be at.

\begin{tabular}{|l|c|c|c|c|}
\hline Gap Resolution Action & Priority & Effort & Timeline & $\begin{array}{c}\text { NRC Vol3 } \\
\text { Crosscut }\end{array}$ \\
\hline $\begin{array}{l}\text { Conduct experimental studies on mechanically } \\
\text { induced or thermally induced molten salt aerosol } \\
\text { formation; Develop and validate models }\end{array}$ & High & High & Near-term & MSR-M3.2 \\
\hline $\begin{array}{l}\text { Conduct experimental studies on salt aerosol } \\
\text { transport and removal mechanisms; Develop and } \\
\text { validate models }\end{array}$ & High & High & Near-term & MSR-M3.2 \\
\hline $\begin{array}{l}\text { Compare experimental molten salt aerosol data to } \\
\text { that of aqueous or sodium aerosols }\end{array}$ & High & Low & Near-term & MSR-M3.2 \\
\hline
\end{tabular}

Tritium Modeling: A sensitivity study related to tritium releases during transient scenarios is recommended as a near-term high priority task. A common question among vendors was related to assessing the importance and impact of tritium releases both as an effluent during normal operation, but also during a transient or accident scenario. Because tritium may be retained in graphite or other materials in the reactor system, it may be advisable to complete a study on the potential for a larger release of tritium during a temperature excursion or other transient. This can be conservatively approximated first with a bounding estimate by using reactor physics tools to calculate the tritium inventory at equilibrium in the reactor, and assuming some over time is trapped or stored somewhere. Other parameters affecting the build-up and release of tritium during transients can be analyzed to assess the impact on tritium releases, and thus provide evidence on the need to incorporate tritium-specific models within severe accident analysis codes.

\begin{tabular}{|l|c|c|c|c|}
\hline Gap Resolution Action & Priority & Effort & Timeline & $\begin{array}{c}\text { NRC Vol3 } \\
\text { Crosscut }\end{array}$ \\
\hline $\begin{array}{l}\text { Evaluate the impact of tritium releases during } \\
\text { transient scenarios with sensitivity studies and } \\
\text { inventory calculations }\end{array}$ & High & Low & Near-term & $\begin{array}{c}\text { FHR-A2/D2, } \\
\text { MSR-CA4 }\end{array}$ \\
\hline
\end{tabular}

Auxiliary System Modeling: The final near-term high priority task is the development of a tool to track the source term in the reactor's auxiliary systems such as an off-gas system. Such a tool can help track the inventory of radionuclides as it transports from each location in various reactor systems that are being proposed as part of MSR designs. Because MSRs utilize a liquid fuel, an off-gas system is a very common proposed system, but evidence from MSRE indicates a large portion of radioactivity transports throughout the off-gas system, often depositing in various locations within the custom-designed system. This represents an important area of source term analysis for MSRs, as potential releases of volatile radionuclides pose a considerable threat to safety.

\begin{tabular}{|l|c|c|c|c|}
\hline Gap Resolution Action & Priority & Effort & Timeline & $\begin{array}{c}\text { NRC Vol3 } \\
\text { Crosscut }\end{array}$ \\
\hline $\begin{array}{l}\text { Develop modeling tools which track radionuclide } \\
\text { flows throughout various stages of a custom } \\
\text { auxiliary system }\end{array}$ & High & Low & Near-term & \\
\hline
\end{tabular}




\subsection{SFR}

As outlined in Section 2.3, the SFR long-term objectives primarily focus on completing or enhancing existing phenomena models, while also improving the validation case. Therefore, many of the near-term tasks are associated with assessing potential extensions to existing models or evaluating available data for adequacy as part of model validation exercises.

Radionuclide Migration and Release from Failed Fuel Pins: Two near-term tasks were identified for potential improvement to in-pin radionuclide migration and ex-pin radionuclide release modeling. As data-driven approaches currently exist, the two near-term tasks examine the potential to use of existing high-fidelity fuel performance codes for the assessment of radionuclide behavior. Such capabilities could help reduce uncertainties and the limitations/conservatisms required with current methods. As the initial step of the MST analysis, increased accuracy propagates through remaining phases of the analysis.

\begin{tabular}{|l|c|c|c|c|}
\hline Gap Resolution Action & Priority & Effort & Timeline & $\begin{array}{c}\text { NRC Vol3 } \\
\text { Crosscut }\end{array}$ \\
\hline $\begin{array}{l}\text { Assessment of high-fidelity fuel performance codes } \\
\text { for the analysis of in-pin radionuclide migration } \\
\text { during pre-transient irradiation }\end{array}$ & Med & Low & Near-term & SFR-M1.2 \\
\hline $\begin{array}{l}\text { Assessment of high-fidelity fuel performance codes } \\
\text { for the analysis of in-pin and ex-pin radionuclide } \\
\text { relocation during transient scenarios. }\end{array}$ & Med & Low & Near-term & SFR-M1.2 \\
\hline
\end{tabular}

Radionuclide Behavior in the Sodium Pool: Two near-term tasks were identified for the assessment of radionuclide behavior within the sodium pool. The only high-priority near-term SFR task is the completion of ongoing NEUP bubble transport experiments for the validation existing models. As bubble transport represents a possible bypass mechanism of radionuclide retention within the sodium pool, the development of a defensible modeling approach for the phenomenon is a necessity for SFR MST analysis. The second task examines the readiness of the current SFR thermodynamic database, developed as part of past DOE SFR MST efforts [23], for its readiness to be directly integrated into MST modeling tools, such as MELCOR.

\begin{tabular}{|l|c|c|c|c|}
\hline Gap Resolution Action & Priority & Effort & Timeline & $\begin{array}{c}\text { NRC Vol3 } \\
\text { Crosscut }\end{array}$ \\
\hline $\begin{array}{l}\text { Completion of NEUP bubble transport in sodium } \\
\text { and lead experimental efforts and validation of } \\
\text { bubble transport models }\end{array}$ & High & Low & Near-term & SFR-M1.3 \\
\hline $\begin{array}{l}\text { Assess the readiness of the current SFR } \\
\text { thermodynamic database for direct integration into } \\
\text { source term modeling tools. }\end{array}$ & Med & Low & Near-term & SFR-M1.3 \\
\hline
\end{tabular}

Radionuclide Aerosol and Vapor Behavior: Three near-term tasks were identified regarding the behavior of radionuclide aerosols and vapors, specifically those associated with sodium. The first task centers on the collection and assessment of sodium aerosol data for the validation of existing models. This work has already begun as part of validation efforts for the SRT code in support of the Versatile Test Reactor (VTR) safety basis [24]. The second task examines whether there is sufficient data available to support the development of pathway plugging by sodium oxides 
through the adaptation of existing plugging models. Lastly, in support of ongoing sodium fire modeling efforts, available data regarding radionuclide release as part of the fire is to be collected and assessed as part of inclusion within the fire models.

\begin{tabular}{|l|c|c|c|c|}
\hline Gap Resolution Action & Priority & Effort & Timeline & $\begin{array}{c}\text { NRC Vol3 } \\
\text { Crosscut }\end{array}$ \\
\hline $\begin{array}{l}\text { Assessment of available data on sodium aerosol } \\
\text { behavior and publication of model validation } \\
\text { efforts. }\end{array}$ & Med & Low & Near-term & SFR-M1.5 \\
\hline $\begin{array}{l}\text { Assessment of available models for the analysis of } \\
\text { pathway plugging due to sodium aerosols and } \\
\text { vapors. }\end{array}$ & Med & Low & Near-term & SFR-M1.5 \\
\hline $\begin{array}{l}\text { Assessment of available data on radionuclide release } \\
\text { from sodium fires }\end{array}$ & Med & Low & Near-term & $\begin{array}{c}\text { SFR-M1.5 } \\
\text { and M1.6 }\end{array}$ \\
\hline
\end{tabular}

For LFRs, there is direct overlap between several of the tasks outlined above. In addition to sodium experiments, there are also lead bubble and vaporization tests being performed under the NEUP program that will support analogous lead pool modeling activities. Similarly, the models adapted for sodium aerosol and vapor transport can be adapted for those forms associated with LFRs, to the extent available data can support.

\subsection{Consequence Modeling}

Near-Field Dispersion: Near-field modeling improvements would include modifications to decrease the conservatism in the near-field, within 500 meters of the source. Furthermore, comparisons would be made between multiple atmospheric transport and dispersion codes, to include Atmospheric Relative Concentrations in Building Wakes (ARCON96), Program for the Meteorological Evaluation of Non-Routine Releases from Nuclear Power Stations (PAVAN), Quick Urban and Industrial Complex (QUIC), and American Meteorological Society / Environmental Protection Agency Regulatory Model (AERMOD), to facilitate licensing applications utilizing these tools.

\begin{tabular}{|l|c|c|c|c|}
\hline Gap Resolution Action & Priority & Effort & Timeline & $\begin{array}{c}\text { NRC Vol3 } \\
\text { Crosscut }\end{array}$ \\
\hline $\begin{array}{l}\text { Improving atmospheric transport and dispersion } \\
\text { modeling to be less conservative for near-field } \\
\text { applications. }\end{array}$ & High & Med & Near-term & TN-CA1 \\
\hline $\begin{array}{l}\text { Benchmark against higher fidelity codes (or } \\
\text { current regulator approved codes) for atmospheric } \\
\text { dispersion }\end{array}$ & High & Med & Near-term & TN-CA1 \\
\hline
\end{tabular}

Isotopic Inventory: In order to characterize the magnitude and timing of the release and facilitate the translation of MELCOR data into use by MACCS, an accurate description of the isotopic inventory within the reactor is necessary. This isotopic inventory should take into account the unique chemical makeup of that specific reactor design, so that when MST codes are applied in MelMACCS to generate the source terms for use in MACCS, the release fractions are applied to an accurate assessment of the radionuclides contained within that system. This item was rated as high priority and near-term because characterizing the isotopic inventory is a prerequisite for 
follow-on activities, to include assessment of activation, released radionuclides, and released chemical forms.

Additionally, inventories containing new radionuclides and associated chemical forms should be assessed. Current MST codes and calculations have focused on LWR technologies, but for advanced reactors these same assumptions are no longer applicable. This task would involve analyzing which additional radionuclides, and associated chemical forms, would potentially require inclusion in advanced reactor consequence analysis modeling, and provided tables of suggested radionuclides and chemical forms for inclusion in advanced reactor consequence analysis.

\begin{tabular}{|l|c|c|c|c|}
\hline Gap Resolution Action & Priority & Effort & Timeline & $\begin{array}{c}\text { NRC Vol3 } \\
\text { Crosscut }\end{array}$ \\
\hline $\begin{array}{l}\text { Provide pre-loaded isotopic inventory files based } \\
\text { on depletion analysis for the specific reactor type } \\
\text { and core geometry of interest. }\end{array}$ & High & Med & Near-term & $\begin{array}{c}\text { SFR-CA2.1, } \\
\text { HTGR- } \\
\text { CA2.2, MSR- } \\
\text { CA2.3, FHR- } \\
\text { CA2.4 }\end{array}$ \\
\hline $\begin{array}{l}\text { Assessing radionuclides and associated chemical } \\
\text { forms needed for inclusion in advanced reactor } \\
\text { consequence analysis. }\end{array}$ & High & Med & Near-term & $\begin{array}{c}\text { SFR-CA2.1, } \\
\text { HTGR- } \\
\text { CA2.2, MSR- } \\
\text { CA2.3, FHR- } \\
\text { CA2.4 }\end{array}$ \\
\hline
\end{tabular}




\section{Conclusion}

In support of the advanced reactor community, a research and development pathway has been established for the creation of comprehensive MST mod/sim capabilities. The pathway and its associated objectives and tasks are purposefully designed to complement the ongoing activities of the NRC's code development program while also addressing key industry needs based on direct vendor input. The established long-term objectives will be used as planning guidance for the research project, with the identified critical near-term tasks providing direction for immediate efforts. Completion of the pathway will require coordination and efforts beyond the MST research project and the following subsection highlights some of the key interfaces to other programs.

\subsection{Cross-cutting Initiatives}

Source term analysis is inherently cross-cutting in nature, given its dependence on transient scenario modeling and the plethora of phenomena involved in radionuclide transport analysis. As part of the current work, an effort was made to identify key areas requiring coordination with other DOE-NE programs or technical areas and the NRC. While the following information is not meant to be comprehensive in nature, it provides an overview of important areas of ongoing or future collaboration.

The recent signing of Addendum No. 3 to the MOU between the NRC and DOE [1] provides an example of a cross-cutting initiative with the goal of improving confidence in MST calculations and a shared alignment of models and research for the regulator and the advanced reactor industry. As mentioned, the NRC has outlined its vision and strategy for MST tool development in ref [2], including the use of SCALE, MELCOR, and MACCS, which is supported by development groups at SNL and ORNL. The research tasks outlined in the current report complement the scheduled NRC research tasks and the data needs identified in the NRC plan, consistent with the DOE-NRC MOU. In particular, the NRC MST model development activities placed a high value on coordination with the DOE NEAMS program on areas of analytical and experimental research and the utilization of NEAMS data, NEAMS benchmarking results, and NEAMS model development to supplement ongoing NRC MST efforts.

In regard to reactor-specific cross-cutting initiatives, the important role of TRISO fuel behavior as part of HTGR (and FHR) MST analyses highlights the critical need of coordination in this area. This includes interfacing with TRISO experimental programs being performed under the Advanced Gas Reactor (AGR) fuel program, in addition to TRISO model development using BISON, which is taking place in the NEAMS fuel performance area. Together, it is possible to develop a thorough basis for TRISO fuel not only from a fuel performance perspective but also in support of MST calculations.

There are many cross-cutting research programs that can be leveraged in support of MST development for MSRs and FHRs. The dependencies on salt chemistry and the physical behavior of certain phases in or out of molten salts, such as corrosion and solid deposition, bubble transport, and aerosol formation, are inherently cross-cutting with reactor design. As such, many complementary activities have already been initiated under DOE-NE's ART MSR Campaign, such as ongoing salt spill experiments, research into prospective off-gas system and their 
potential source term, and molten salt qualification efforts. Additionally, the MSR Campaign has supported activities in analyzing initiating events and associated licensing basis events for MSRs, as well as currently ongoing PIRT activities, thereby providing a basis of understanding for accident scenario modeling. Similarly, efforts of the DOE-funded Center for Molten Salts in Extreme Environments (MSEE) and Office of Energy Efficiency \& Renewable Energy programs on thermal storage also offer potential areas of collaboration.

Within the NEAMS program, there are several cross-cutting MSR/FHR initiatives as well. Due to the highly multiphysics nature of MSRs, many ongoing modeling and simulation efforts will provide insight on radionuclide transport within the reactor system and provide information which can be used as input or benchmarking for severe accident analysis codes such as MELCOR. An example is the current efforts under NEAMS in the development of chemistry modeling tools for MSRs, including thermochemical and thermogalvanic phenomena. Similarly, the ongoing development of the Mole code, an engineering scale MSR species transport code [25], which can provide multiphysics insights through coupling with other NEAMS tools.

For SFRs, an important area of ongoing collaboration is with bubble transport experiments taking place under the NEUP program. These efforts are providing critical validation data for a key phenomenon and providing the basis for an international benchmark exercise. Data on in-pin radionuclide behavior comes primarily from historic DOE post-irradiation examination (PIE) and is being continually improved with new information from the Advanced Fuels Campaign (AFC). In addition, BISON and SAS4A offer potential avenues to increase mechanistic modeling of inpin radionuclide behavior. Lastly, the MST code validation efforts ongoing in support of the VTR program and safety basis development highlights the importance of the tasks outlined here and is a close point of project coordination.

Finally, regarding consequence analysis, cross-cutting initiatives exist among each of the advanced reactor types, as many of the concepts for advanced reactors are independent of the specific reactor type. Enhancements in near-field modeling capabilities, development of models for unique deposition behavior, refinement of dose conversion factors, enhancements in decontamination modeling, and simplification methods for multi-unit consequence assessments are common among each of the advanced reactor types and therefore represent an opportunity to leverage work amongst multiple programs. Furthermore, although the specifics of isotopic inventories and potential chemical hazards depend on the particular advanced reactor type, the pathway to resolve these gaps is common and therefore represents a cross-cutting approach as well. 


\section{Bibliography}

[1] "Memorandum of Understanding Between Department of Energy and Nuclear Regulatory Commission on Nuclear Energy Innovation - Radiological Source Term and MELCOR Collaboration, Addendum No. 3," DOE/NRC, April 292021.

[2] "NRC Non-Light Water Reactor (Non-LWR) Vision and Strategy, Volume 3 - Computer Code Development Plans for Severe Accident Progression, Source Term, and Consequence Analysis, Draft Rev.1," U.S. Nuclear Regulatory Commission, ML20030A178, January 312020.

[3] N. C. Andrews, A. J. Clark, T. M. Nenoff, J. E. Leute, and D. L. Luxat, "Mechanistic Source Term Considerations for Advanced Non-LWRs," Sandia National Laboratories, SAND2020-6730, July 2020.

[4] S. Shahbazi and D. Grabaskas, "Functional Requirements for the Modeling and Simulation of Advanced (Non-LWR) Reactor Mechanistic Source Term," Argonne National Laboratory, ANL/NSE-20/17, June 2020.

[5] S. Shahbazi et al., "Survey and Assessment of Computational Capabilities for Advanced (Non-LWR) Reactor Mechanistic Source Term Analysis," Argonne National Laboratory and Sandia National Laboratories, ANL/NSE-20/39, SAND2021-3250R, September 2020.

[6] D. Grabaskas et al., "Regulatory Technology Development Plan - SFR Mechanistic Source Term Trial Calculation," Argonne National Laboratory, ANL-ART-49, October 2016.

[7] J. Leute and K. Wagner, "A Pathway for the Development of Advanced Reactor Mechanistic Source Term Modeling and Simulation Capabilities for High Temperature Gas Reactors and Consequence Analysis," DRAFT, 2021.

[8] S. J. Ball and S. E. Fisher, "Next Generation Nuclear Plant Phenomena Identification and Ranking Tables (PIRTs) Volume 1: Main Report," Oak Ridge National Laboratory, NUREG/CR-6944, Vol. 1; ORNL/TM-2007/147, Vol. 1, March 2008.

[9] J. Jerden, D. Grabaskas, and M. Bucknor, "Development of a mechanistic source term approach for liquid-fueled Molten Salt Reactors," Argonne National Laboratory, ANL/CFCT-19/4, July 2019.

[10]S. Thomas and J. Jerden, "Mechanistic Source Term Development for Liquid Fueled MSRs - Model Development Update," Argonne National Laboratory, ANL/CFCT-20/16, July 2020.

[11]D. Grabaskas, A. J. Brunett, M. D. Bucknor, J. J. Sienicki, and T. Sofu, "Regulatory Technology Development Plan - SFR Mechanistic Source Term Development," Argonne National Laboratory, ANL-ART-3, February 2015.

[12]D. Grabaskas, M. D. Bucknor, and J. L. Jerden, "Regulatory Technology Development Plan - SFR Mechanistic Source Term Metal Fuel Radionuclide Release," Argonne National Laboratory, ANL-ART-38, February 2016. 
[13]J. Ard et al., "FY20 Status report on the Molten Salt Thermodynamic Database (MSTDB) development," Oak Ridge National Laboratory, ORNL/SPR-2020/1648, September 2020.

[14]J. W. McMurray, T. M. Besmann, J. Ard, S. Utlak, and R. A. Lefebvre, "Status of the molten salt thermodynamic database, MSTDB," Oak Ridge National Laboratory, ORNL/SPR-2019/1208, August 2019.

[15]J. W. McMurray et al., "Roadmap for thermal property measurements of Molten Salt Reactor systems," ORNL/SPR-2020/1865, March 2021.

[16]P. Bajpai, M. Poschmann, D. Andrš, C. Bhave, M. Tonks, and M. Piro, "Development of a New Thermochemistry Solver for Multiphysics Simulations of Nuclear Materials," in TMS 2020 149th Annual Meeting \& Exhibition Supplemental Proceedings(The Minerals, Metals \& Materials Series, 2020, pp. 1013-1025.

[17]M. H. A. Piro, S. Simunovic, T. M. Besmann, B. J. Lewis, and W. T. Thompson, "The thermochemistry library Thermochimica," Computational Materials Science, vol. 67, pp. 266-272, 2013.

[18]M. H. A. Piro, S. Simunovic, T. M. Besmann, and M. Poschmann, "Thermochimica User Manual," 2019, Available: https://github.com/ORNL-CEES/thermochimica, Accessed on: September 1, 2020.

[19]M. Poschmann, B. W. N. Fitzpatrick, S. Simunovic, and M. H. A. Piro, "Recent Development of Thermochimica for Simulations of Nuclear Materials," in TMS 2020 149th Annual Meeting \& Exhibition Supplemental Proceedings, 2020, pp. 1003-1012.

[20]S. Shahbazi, S. Thomas, A. Merwin, Q. Zhou, and M. Bucknor, "Thermochemical Modeling in Molten Fluoride Salts for Radionuclide Speciation," Argonne National Laboratory, ANL/KP-21/1, KP-RPT-000139, February 2021.

[21]D. E. Holcomb et al., "Early Phase Molten Salt Reactor Safety Evaluation Considerations," Oak Ridge National Laboratory, ORNL/TM-2020/1719, September 2020.

[22]F. J. Peretz, "Identification and Evaluation of Alternatives for the Disposition of Fluoride Fuel and Flush Salts from the Molten Salt Reactor Experiment at ORNL," ORNL/ER-380, August 1996.

[23]D. Grabaskas et al., "Regulatory Technology Development Plan - Sodium Fast Reactor: Mechanistic Source Term Development - Trial Calculation," Argonne National Laboratory,, ANL-ART-49, 2016.

[24]D. Grabaskas, J. Jerden, and M. Bucknor, "A New Computational Tool for Rapid Uncertainty and Sensitivity Analyses of Sodium Reactor Source Term," presented at the 2019 ANS Annual Meeting, 2019.

[25]A. Graham, R. R. Pillai, B. S. Collins, and J. W. McMurray, "Engineering scale molten salt corrosion and chemistry code development," ORNL/SPR-2020/1582, July 2020. 
[26]W. Moe, J. Kinsey, K. Perry, D. Petti, and G. Gibbs, "Mechanistic Source Term White Paper," in "Next Generation Nuclear Plant Project," Idaho National Laboratory, INL/EXT10-17997, July 2010.

[27]F. Bostelmann, C. Celik, M. L. Williams, R. J. Ellis, G. Ilas, and W. A. Wieselquist, "SCALE capabilities for high temperature gas-cooled reactor analysis," Annals of Nuclear Energy, vol. 147, 2020.

[28]Y. Xu, H. Li, F. Xie, J. Cao, and J. Tong, "Source Term Analysis of Tritium in HTR-10," Fusion Science and Technology, vol. 71, no. 4, pp. 671-678, 2017.

[29]H. D. Gougar et al., "The US Department of Energy's high temperature reactor research and development program - Progress as of 2019," Nuclear Engineering and Design, vol. $358,2020$.

[30]P. A. Demkowicz, B. Liu, and J. D. Hunn, "Coated particle fuel: Historical perspectives and current progress," Journal of Nuclear Materials, vol. 515, pp. 434-450, 2019.

[31]C. W. Forsberg, J. D. Stempien, M. J. Minck, and R. G. Ballinger, "Understanding and Pathways to Avoid Major Fuel Failures and Radionuclide Releases in Fluoride SaltCooled High-Temperature Reactor Severe Accidents," Nuclear Technology, vol. 194, no. 3, pp. 295-313, 2016.

[32]J. B. Malherbe, "Diffusion of fission products and radiation damage in SiC," Journal of Physics D: Applied Physics, vol. 46, no. 47, 2013.

[33]"Fuel Performance and Fission Product Behaviour in Gas-Cooled Reactors," IAEA, Vienna, Austria, IAEA-TECDOC-978, November 1997.

[34]R. Moormann, "Fission Product Transport and Source Terms in HTRs: Experience from AVR Pebble Bed Reactor," Science and Technology of Nuclear Installations, vol. 2008, pp. 1-14, 2008.

[35]R. Moormann, "AVR prototype pebble bed reactor: a safety re-evaluation of its operation and consequences for future reactors," Kerntechnik, vol. 74, no. 1-2, pp. 8-21, 2009.

[36]P. Humrickhouse, "HTGR Dust Safety Issues and Needs for Research and Development," Idaho National Laboratory, INL/EXT-11-21097, June 2011.

[37] "Risk-Informed Performance-Based Technology Inclusive Guidance for non-LWR Licensing Basis Development, Revision 1," Nuclear Energy Institute, 18-04, August 2019.

[38]S. A. Walker and W. Ji, "Species transport analysis of noble metal fission product transport, deposition, and extraction in the molten salt reactor experiment," Annals of Nuclear Energy, vol. 158, 2021.

[39]J. E. Leute, B. Beeny, F. Gelbard, and A. Clark, "Identification and Resolution of Gaps in Mechanistic Source Term and Consequence Analysis Modeling for Molten Salt Reactors," Sandia National Laboratories, Albuquerque, NM, In review, April 2021. 
[40]D. Grabaskas, T. Fei, and J. Jerden, "Technical Letter Report on The Assessment of Tritium Detection and Control in Molten Salt Reactors: Final Report," Argonne National Laboratory, ANL/NSE-20-15, May 2020.

[41]P. W. Humrickhouse and T. F. Fuerst, "Tritium Transport Phenomena in Molten-Salt Reactors," Idaho National Laboratory, INL EXT-20-59927, September 2020.

[42]D. Grabaskas, M. Bucknor, and J. Jerden, "Regulatory Technology Development Plan Sodium Fast Reactor: Mechanistic Source Term - Metal Fuel Radionuclide Release," ANL-ART-38, 2016.

[43]D. Grabaskas, M. Bucknor, J. Jerden, T. Starkus, and S. Shahbazi, "Simplified Radionuclide Transport (SRT) Code: Users Manual," ANL-SRT-4, Rev 2.0.1, 2020.

[44]A. Tentner, A. Karahan, and S. Kang, "Overview of the SAS4A Metallic Fuel Models and Extended Analysis of a Postulated Severe Accident in the Prototype Gen-IV SodiumCooled Fast Reactor," Nuclear Technology, vol. 206, no. 2, pp. 242-254, 2020.

[45]S. Novascone, A. Casagranda, P. Medvedev, C. Matthews, and A. Zabriskie, "Summary and Assessment of Metallic Fuel Capabilities in Bison," INL/EXT-18-51399, 2018.

[46] T. H. Bauer, A. Wright, W. Robinson, J. Holland, and E. Rhodes, "Behavior of Modern Metallic Fuel in TREAT Transient Overpower Tests," Nuclear Technology, vol. 92, 1990.

[47] Outotec, "HSC Chemistry 8 User's Guide," 2014.

[48]R. Schram, E. Cordfunke, and M. Huntelaar, "Source Term Calculations of the ALMR," ECN-R-95-021, 1995.

[49]A. Castleman, "LMFBR Safety I, Fission-Product Behavior in Sodium," Nuclear Safety, vol. 11, no. 5, pp. 379-389, 1970.

[50]C. Erdman, J. Kelly, M. Kirbiyik, and A. Reynolds, "Radionuclide Production, Transport, and Release from Normal Operation of Liquid-Metal-Cooled Fast Breeder Reactors," EPA-520/3-75-019, 1975.

[51]University of Wisconsin and Argonne National Laboratory, "NEUP Project 18-14908: Experimental Measurements of Fission Product Retention in Liquid Sodium," 2018.

[52]University of New Mexico, "NEUP Project 18-15471: Integral Experimental Investigation of Radioisotope Retention in Stagnant and Flowing Lead for the Mechanistic Source Term Evaluation of Lead Cooled Fast Reactor " 2018.

[53]K. Becker and M. Anderson, "Experimental Study of SRT Scrubbing Model in Water Coolant Pool," Nuclear Engineering and Design, vol. 377, 2021.

[54]D. Powers, "Aerosol Penetration of Leak Peathways - An Examination of the Available Data and Models," SAND2009-1701, 2009. 
[55]S. Narayanam et al., "Experimental Measurements and Theoretical Simulation of Sodium Combustion Aerosol Leakage through Capillaries," Progress in Nuclear Energy, vol. 118, 2020.

[56]T. Hua et al., "Development of Mechanistic Source Term Analysis Tool SASA4A-FATE for Lead- and Sodium-Cooled Fast Reactors," Nuclear Technology, vol. 206, no. 2, pp. 206-2117, 2020.

[57]A. Alemberti et al., "The Status of the ALFRED Project," IAEA International Conference on Fast Reactors and Related Fuel Cycles - Fast Reactors 21/22, 2021.

[58]D. Clayton and N. E. Bixler, "Assessment of the MACCS Code Applicability for Nearfield Consequence Analysis," Sandia National Laboratories, Albuquerque, NM, SAND20202609, 2020.

[59]D. Clayton, "Implementation of Additional Models into the MACCS Code for Nearfield Consequeunce Analysis," 2021.

[60]J. Fulton and M. Cohen, "Development of a Near-Field Capable HYSPLIT Atmospheric Dispersion and Transport Model," Sandia National Laboratories / National Oceanic Atmospheric Administration, SAND2021-2715O, 2020.

[61]"State-of-the-Art Reactor Consequence Analyses Project Volume 1: Peach Bottom Integrated Analysis," Sandia National Laboratories, NUREG/CR-7110, January 2012.

[62]N. Andrews, M. Higgins, and A. Taconi, "Preliminary Radioisotope Screening for Off-Site Consequence Assessment of Advanced Non-LWR Systems," Sandia National Laboratories, Albuquerque, NM, Draft Report, 2020.

[63]J. E. Leute and N. Bixler, "Simplified Approach to Multi-Unit Probabilistic Risk Assessment (PRA) Proposal," Sandia National Laboratories, Albuquerque, NM, SAND2021-0653, 2021. 


\section{Appendix}

The following appendices outline the detailed gap resolution assessment conducted as part of the current work. A separate appendix is provided for each advanced reactor type and also consequence modeling. Each appendix reviews the gap resolution actions identified, including the rating of the action based on priority, effort, and timeframe, in addition to any identified activities cross-cutting with those proposed in NRC Volume 3, code development vision and strategy [2]. The level definitions used for this effort are outlined in Table 2.

Table 2: Gap Resolution Action - Level Definition

\begin{tabular}{|c|c|c|c|c|}
\hline Metric & \multicolumn{4}{|c|}{ Levels $^{1}$} \\
\hline Priority & $\begin{array}{l}\text { High: } \\
\text { - Major impact on } \\
\text { source term } \\
\text { analysis results } \\
\text { - Potential "show- } \\
\text { stopper" for } \\
\text { completing source } \\
\text { term analyses } \\
\text { Major uncertainty } \\
\text { that is difficult to } \\
\text { bound or has large } \\
\text { impact on system } \\
\text { design }\end{array}$ & $\begin{array}{cc}\text { Medium } \\
\text { - } & \text { Appr } \\
\text { on so } \\
\text { analy } \\
\text { - } \quad \text { Can p } \\
\text { addre } \\
\text { conse } \\
\text { boun } \\
\text { assum } \\
\text { with }\end{array}$ & $\begin{array}{l}\text { iable impact } \\
\text { ce term } \\
\text { s results } \\
\text { ssibly be } \\
\text { ed through } \\
\text { jative or } \\
\text { ng } \\
\text { tions but } \\
\text { awbacks }\end{array}$ & $\begin{array}{l}\text { Low: } \\
\text { - } \quad \text { Minor expected } \\
\text { impact on source } \\
\text { term analysis } \\
\text { results } \\
\text { - Easily addressed } \\
\text { through } \\
\text { conservative or } \\
\text { bounding } \\
\text { assumptions } \\
\text { Modeling gaps that } \\
\text { are mostly resolved } \\
\text { or tools that are } \\
\text { adequate for source } \\
\text { term analyses in } \\
\text { their current form }\end{array}$ \\
\hline Effort & $\begin{array}{l}\text { High: } \\
\text { - Multi-year analysis } \\
\text { or development } \\
\text { effort requiring } \\
\text { significant } \\
\text { technical expertise } \\
\text { Complex } \\
\text { experimentation } \\
\text { needed, such as use } \\
\text { of radioactive } \\
\text { materials or } \\
\text { irradiation facilities }\end{array}$ & $\begin{array}{l}\text { Medium } \\
\text { - } \\
\text { May r } \\
\text { year a } \\
\text { devel } \\
\text { - } \\
\text { Exper } \\
\text { neede } \\
\text { bench } \\
\text { non-r } \\
\text { hazar }\end{array}$ & $\begin{array}{l}\text { quire multi- } \\
\text { alysis or } \\
\text { oment effort } \\
\text { nentation } \\
\text { but at } \\
\text { scale using } \\
\text { lioactive or } \\
\text { materials }\end{array}$ & $\begin{array}{l}\text { Low: } \\
\text { - Less than a year of } \\
\text { analysis or } \\
\text { development effort } \\
\text { required } \\
\text { - } \\
\text { No further } \\
\text { experimentation } \\
\text { needed }\end{array}$ \\
\hline Timeframe & $\begin{array}{l}\text { Near-term: } \\
\text { - Items identified as r } \\
\text { near-term resolution } \\
\text { immediate regulator } \\
\text { facilitate license app } \\
\text { progress, or address } \\
\text { design decisions. }\end{array}$ & $\begin{array}{l}\text { quiring } \\
\text { o address } \\
\text { concerns, } \\
\text { cations in } \\
\text { mportant }\end{array}$ & 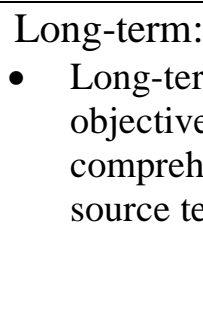 & $\begin{array}{l}\text { m development } \\
\text { s to create } \\
\text { ensive advanced reactor } \\
\mathrm{rm} \text { analysis tools }\end{array}$ \\
\hline
\end{tabular}

${ }^{1}$ The gap resolution actions do not need to meet all bulleted definitions for the selected level. 


\section{A High Temperature Gas Reactors}

The landscape of HTGRs has been evolving from the earliest designs and the DOE NGNP [26] project to designs currently proposed by vendors. There has been considerable research and some operating experience. However, gaps still exist regarding:

- Core neutronics and depletion

- Experiments for radionuclide release and associated models from current generation TRISO particles

- Circulating inventory

- Cross-cutting accident scenario modeling

The following sections are extracted from ref [7] and describe the research gaps, recommended paths to resolution, and an assessment of the priority, level of effort, completion timeline, and cross-reference to NRC non-LWR research plan.

\section{A.1 Core Neutronics and Fuel Depletion}

HTGRs present some unique challenges for core neutronic performance and fuel depletion. For example, pebble bed reactors include multi-burn-up and multi-pass fuel pebbles. Furthermore, the HTGR designs show strong temperature- and fast-to-thermal spectrum coupling effects that require higher fidelity, coupled models. Source term codes like MELCOR require input from these analyses to specify the radionuclide inventory, the power profiles, the decay heat, and the transient power response inputs for their point kinetic models. Faster running core neutronic and fuel depletion codes such as SCALE benefit from benchmarking to high fidelity codes. A related area is activation of He-3 in the working fluid and trace impurities in the graphite that form tritium.

\section{A.1.1 Neutronics models}

Provide models for various generic core geometries for HTGR concepts in NRCsupported code suites such as SCALE:

Recently, ORNL has completed PBMR-400 core neutronic, radionuclide inventory, and decay heat evaluations for an NRC non-LWR demonstration calculation using SCALE [27]. The analysts cited challenges with modeling (a) pebble packing, (b) the strong neutronic and temperature coupling, (c) the radial and axial spectral variations, and (d) moving and multipass, multi-burn-up pebbles. Code comparisons to data and benchmarking with high fidelity codes is important to validate faster running approaches such as those in SCALE.

Source term transient analysis codes like MELCOR rely on input from neutronics models for the point kinetics model. The data inputs include for the dominant feedbacks for the point kinetics models, the decay and abundance values for a range of neutron groups, the prompt neutron generation time and total effective yield, the average neutron yield per fission, the prompt and delayed energy released per fission, and the average and prompt neutron lifetimes. 
The resolution to this gap is application of advanced models for HTGR designs to provide benchmark data for fast-running neutronic and depletion codes and input data for the point kinetic models used in source term codes, such as MELCOR. The priority of these tasks is judged as low due to already ongoing NRC SCALE work to satisfy the immediate needs for NRC Volume 3 demonstration calculations. The benchmarking effort to NEAMS would be a low effort but valuable prior to NRC's specific design reviews. This work can be differed to a long-term timeline.

\begin{tabular}{|l|c|c|c|c|}
\hline Gap Resolution Action & Priority & Effort & Timeline & $\begin{array}{c}\text { NRC Vol3 } \\
\text { Crosscut }\end{array}$ \\
\hline $\begin{array}{l}\text { Provide models for various generic core } \\
\text { geometries for HTGR concepts in NRC- } \\
\text { supported code suites such as SCALE }\end{array}$ & Low & Low & $\begin{array}{c}\text { Long- } \\
\text { term }\end{array}$ & HTGR-M2.1 \\
\hline
\end{tabular}

\section{A.1.2 Activation}

\section{Model the activation of coolant or core components:}

Ref [28] showed that tritium was an important nuclide in the primary loop of the HTR-10 HTGR. Their subsequent analysis and modeling show that the majority of total tritium was produced by ternary fission reactions. However, their analysis assumed no releases from intact TRISO and therefore, only broken TRISO particles released tritium into the primary loop. Therefore, the primary source of tritium in the primary loop comes from the activation of trace amounts of He-3 in the working fluid and Li-6 impurities in the graphite. The key pathways of the tritium during normal operations were removal by the helium purification system, permeation to the secondary loop, primary system leakage pathways, and natural decay. Key concerns in accident conditions include release from helium purification system and release from graphite structures at elevated temperatures.

The resolution to this gap is development of tritium behavior models such as those identified by ref [28]. The priority and effort are judged as low for transient source term analysis, although important for operational evaluations. The timeline is assigned to long-term due to the low priority.

\begin{tabular}{|l|c|c|c|c|}
\hline Gap Resolution Action & Priority & Effort & Timeline & $\begin{array}{c}\text { NRC Vol3 } \\
\text { Crosscut }\end{array}$ \\
\hline $\begin{array}{l}\text { Model the activation of coolant or core } \\
\text { components }\end{array}$ & Low & Low & $\begin{array}{c}\text { Long- } \\
\text { term }\end{array}$ & HTGR-M2.2 \\
\hline
\end{tabular}

\section{A.2 TRISO Fuel Particle Releases}

Historical HTGR experience is largely based on uranium oxide fuels. Consequently, much of the corresponding experimental data was for radionuclide diffusion from uranium oxide TRISO. However, most new manufacturers have improved the technology using UCO fuel kernels. As the TRISO materials and manufacturing techniques change, there is a need for new radionuclide diffusivity and release data. In addition, accurately capturing the failure rate of TRISO particles and initial failure fraction within any software suite is of high priority, which may be batch- and manufacturer-dependent. The effect of burnup on the release and failure 
characteristics of TRISO fuel particles as well as the temperature-dependent behaviors are key to predicting the TRISO source term.

The DOE is supporting research and development of advanced reactor systems through the Advanced Reactor Technologies (ART) Program. The goal of the program is to identify and reduce the technical risks confronting the deployment of non-water-cooled reactors. The gascooled reactor (ART-GCR) campaign continues with activities in fuel development and qualification, graphite characterization and qualification, high-temperature alloys qualification, and core analysis methods development and experimental validation. ART-GCR works with industrial vendors to help them achieve their deployment goals. In addition to the basic qualification programs, the ART program solicits input from reactor and fuel vendors on technical issues and research priorities, including developing and testing next generation TRISO fuel, advanced graphite grades, and design and high-fidelity core analysis tools [29].

The advanced code TRISO radionuclide release models include advanced calculations with many data and experimental validation needs. Similarly, source term transient analysis codes like MELCOR also rely on input from experiments or first principles models for the radionuclide release from the fuel particles. MELCOR includes a general Booth-type diffusion radionuclide model but relies on fuel-specific inputs for the Arrhenius equation inputs. Furthermore, MELCOR's TRISO diffusivity models require radionuclide-specific and TRISO layer-specific diffusivities. Consequently, there are broad data needs to characterize radionuclide release from modern TRISO fuel across a broad range of temperatures.

The advanced code TRISO models also include thermo-mechanical failure calculations with many data and experimental validation needs. Fuel-specific data is needed to develop and benchmark the TRISO failure models. MELCOR includes considerable flexibility for the TRISO failure model that would typically be in the form of an empirical correlation. For example, the existing $\mathrm{UO}_{2}$ TRISO failure model is an exponential curve fit for failure versus temperature from German post-irradiation thermal testing. The results from experiments or advanced code calculations are needed to develop an empirical TRISO UCO failure model. Several tasks are envisioned to address this research need.

\section{A.2.1 TRISO release report}

\section{Summarize existing data on radionuclide releases from TRISO fuel particles:}

First, HTGR vendors requested a summary of the currently available data. This first task would catalog TRISO radionuclide release and failure data from past experimental tests. As documented and available, an assessment would be made on the quality assurance practices applied during to the experimental research. The report would specifically highlight modern UCO TRISO data versus older uranium-oxide fueled TRISO tests. The report would summarize the findings, overlapping measurements, and gaps. The path to resolution is a report summarizing the literature review of available TRISO radionuclide release data and an identification of gaps.

Discussions with vendors identified this task as high priority. The compilation of available TRISO radionuclide release and failure data is identified as a low effort, near-term task. 


\begin{tabular}{|l|c|c|c|c|}
\hline Gap Resolution Action & Priority & Effort & Timeline & $\begin{array}{c}\text { NRC Vol3 } \\
\text { Crosscut }\end{array}$ \\
\hline $\begin{array}{l}\text { Summarize existing data on radionuclide releases } \\
\text { from TRISO fuel particles }\end{array}$ & High & Low & $\begin{array}{c}\text { Near- } \\
\text { term }\end{array}$ & HTGR-M2.1 \\
\hline
\end{tabular}

\section{A.2.2 TRISO release models}

\section{Develop TRISO release models for severe accident analysis codes:}

The second task focuses on radionuclide release modeling and benchmarks. MELCOR includes a general-purpose radionuclide release model but would benefit from benchmarking with NEAMS codes. The benchmarking results may identify requirements for model improvement or development (e.g., a UCO TRISO failure model). The path to resolution is continuation of vendor-specific post-irradiation TRISO release and failure testing (see Section A.2.3), benchmarking with NEAMS codes, and development of empirical correlations for UCO TRISO failure from experiments of NEAMS code results.

Similar to the previous resolution, discussions with industry identified this task as high priority. Benchmarking to UCO TRISO results from NEAMS codes and development of an empirical UCO TRISO failure model could start immediately but is expected to be a long-term task that is dependent on long-term experimental programs. A medium level of effort is estimated due to the long-term timeline and uncertainties in the level of effort for the code development to close gaps identified during the benchmarks.

\begin{tabular}{|l|c|c|c|c|}
\hline Gap Resolution Action & Priority & Effort & Timeline & $\begin{array}{c}\text { NRC Vol3 } \\
\text { Crosscut }\end{array}$ \\
\hline $\begin{array}{l}\text { Development and benchmarking of TRISO } \\
\text { release models }\end{array}$ & High & Med & $\begin{array}{c}\text { Long- } \\
\text { term }\end{array}$ & HTGR-M2.1 \\
\hline
\end{tabular}

\section{A.2.3 TRISO release experiments}

\section{Continue TRISO irradiation and release experiments:}

DOE has planned and is performing TRISO irradiation experiments to confirm the release behavior to validate the models outlined above in Section A.2.1. The new data is important to characterize the improved margins of modern TRISO fuels and improved manufacturing techniques. Historical data covers different fuel kernel types, different manufacturing quality assurance, a limited temperature range characterization, and a limited radionuclide release characterization (e.g., primarily $\mathrm{Kr}, \mathrm{Xe}, \mathrm{Cs}, \mathrm{Sr}$, and Ag). The TRISO failure characteristics of $\mathrm{UO}_{2}$ versus UCO fuel are also very different [30]. Consequently, the applicability of historical data is limited for new reactor designs. Finally, with uncertainties of the HTGR peak fuel temperatures profiles for the LBEs, testing to TRISO failure is important to identify safety margins and to develop TRISO failure correlations. The resolution to this gap is prototypical experiments for radionuclide release from modern TRISO fuels [29].

Due to the cost and long duration of experimental work, this task is classified as high effort on a long-term timeline. The TRISO experimental release experiments have a very high priority due to the limited to non-existent data on modern UCO TRISO release and failure data. The experimental data is essential to develop a strong technical basis for modern TRISO fuel and 
the associated regulatory acceptance. Task A.2.1 supports this task by identifying known gaps in the available experimental data.

\begin{tabular}{|l|c|c|c|c|}
\hline Gap Resolution Action & Priority & Effort & Timeline & $\begin{array}{c}\text { NRC Vol3 } \\
\text { Crosscut }\end{array}$ \\
\hline $\begin{array}{l}\text { Continued TRISO irradiation and release } \\
\text { experiments }\end{array}$ & High & High & $\begin{array}{c}\text { Long- } \\
\text { term }\end{array}$ & HTGR-M2.1 \\
\hline
\end{tabular}

\section{A.2.4 Core heat transfer}

\section{Obtain heat transfer data for fuel from experimental research programs; benchmark models in MELCOR against high fidelity codes:}

The key safety aspects of HTGRs are the TRISO fuel design and passive cooling. The geometry of the proposed designs does not include in-vessel natural convection pathways for cooling like LWR designs but rather rely on conduction and radiation from the fuel to the reflectors and the vessel boundary. The effectiveness of the heat dissipation and passive cooling is dependent on the radial and axial heat transfer from the prismatic fuel compacts or pebble beds to the surrounding graphite reflectors. The complicating factors for pebble bed designs is determining the combined radiation and convection in a pebble bed. The complicating factors for prismatic designs are the gaps between the fuel compacts. All designs are impacted by the thermophysical properties of the fuel and reflectors, particularly the impact of impact of irradiation and temperature on the graphite conductivity.

Source term transient analysis codes like MELCOR include effective heat transfer correlations of simultaneous conduction and radiation in a porous media for pebble bed reactors and the effective radial conductivity and gap conductance models for prismatic designs. These correlations are used to predict high temperature radial and axial heat transfer from the fuel to the reflectors. However, the correlations are dependent on a number of inputs to characterize the thermal-physical properties, the pebble bed porosity, the graphite emissivity, and the gap conductance. There are similar overlapping data needs for detailed codes.

The resolution is to this gap contains two components. First, design-specific, irradiated graphite and UCO thermophysical properties and heat transfer data should be obtained from ongoing experimental research programs. The second subtask would be a benchmark of MELCOR's empirical heat transfer models to high-fidelity NEAMS calculations.

Given the importance of temperature on the magnitude of the TRISO release, this task is identified as medium importance. The NRC HTGR PIRT [8] identified the thermophysical properties of graphite as having high importance with a low to medium level of knowledge. Furthermore, the previous graphite grade qualified for nuclear service in the U.S. is no longer available. Hence, it will be necessary to qualify new grades of graphite for use in HTGRs and their irradiated properties. The timeframe is judged as long-term due to experimental schedules and an associated delay for NEAMS benchmark data. The effort of the data incorporation and benchmark comparison is low. 


\begin{tabular}{|l|c|c|c|c|}
\hline Gap Resolution Action & Priority & Effort & Timeline & $\begin{array}{c}\text { NRC Vol3 } \\
\text { Crosscut }\end{array}$ \\
\hline $\begin{array}{l}\text { Obtain heat transfer data for fuel from } \\
\text { experimental research programs; benchmark } \\
\text { models in MELCOR against high fidelity codes }\end{array}$ & Med & Low & $\begin{array}{c}\text { Long- } \\
\text { term }\end{array}$ & HTGR-M2.1 \\
\hline
\end{tabular}

\section{A.3 Radionuclide Transport in Core Materials}

As a companion to Section A.2, the research gaps in this section documents other aspects of radionuclide transport through the core materials and the impact of graphite oxidation. These items are discussed in the following subsections.

\section{A.3.1 Radionuclide transport and oxidation behavior report}

Summarize existing data on radionuclide absorption into graphite, radionuclide carbide formation, and graphite oxidation:

This near-term task would summarize the current understanding of radionuclide absorption into graphite and carbide formation. It will also summarize the state of knowledge of graphite oxidation with steam and air. The review will identify gaps and research needs for long-term experimental and model development. This task compliments efforts identified in Section A.2.1 for more complex interactions of radionuclides. Radionuclide absorption and carbide formation processes are important processes that change the characteristics of the source term by impacting the timing and magnitude of the releases. Similarly, a survey of graphite oxidation research and associated gas generation has a direct impact of the thermal response and degradation of the fuel and ex-vessel combustion potential. The task will support the long-term research identified in Section A.3.2 to identify data and modeling gaps. A medium priority is assigned due to the importance of this task to document the current state-ofknowledge and support long-term research. A near-term and low level of effort is assigned for this literature and research survey that proceeds subsequent long-term tasks.

\begin{tabular}{|l|c|c|c|c|}
\hline Gap Resolution Action & Priority & Effort & Timeline & $\begin{array}{c}\text { NRC Vol3 } \\
\text { Crosscut }\end{array}$ \\
\hline $\begin{array}{l}\text { Summarize existing data on radionuclide } \\
\text { absorption into graphite, radionuclide carbide } \\
\text { formation, and graphite oxidation. }\end{array}$ & Med & Low & Near-term & HTGR-M2.1 \\
\hline
\end{tabular}

\section{A.3.2 Radionuclide transport}

Research and model development on the transport of radionuclides in core materials: The prediction of radionuclide transport from the TRISO particle to the working fluid includes many complicated phenomena such as chemical reactions, sorption, and diffusion transport across various materials such as the surrounding graphite matrix. The transport processes are also impacted by irradiation and the media temperature. Forsberg et al. has identified the important radionuclides from the perspective of health importance or their higher transmittance through the TRISO silicon-carbon layer [31]. Of the nine elements identified, $\mathrm{Ag}$ and $\mathrm{Pd}$ are the only elements that are released through intact $\mathrm{SiC}$ and graphite. The other seven elements $(\mathrm{Cs}, \mathrm{Ce}, \mathrm{Eu}, \mathrm{I}, \mathrm{Kr}, \mathrm{Sr}$, and $\mathrm{Xe}$ ) require broken $\mathrm{SiC}$ for any significant release. Furthermore, Forsberg identifies retardation of release by adsorption on graphite or carbon for five elements 
(Cs, I, Kr, Sr, Xe). Finally, three elements (Ce, Eu, Sr) react with carbon to form carbides. However, the data is sparse, often conflicting, and limited for new TRISO fuels. Experimental data and corresponding models are needed to characterize the behavior of the diffusion transport, adsorption into carbon, and carbide formation.

Supporting the need for continued experimental research, Malherbe has provided a comprehensive summary of the radionuclide diffusivities in TRISO layers [32]. Their work shows that the reported diffusivities at the same temperature can vary by orders of magnitude among different researchers. Other research has shown the importance of adsorption of metallic radionuclides (e.g., Sr) into the relative porous carbon matrix. The models and data for this behavior are limited and dependent on many factors including graphite composition and porosity, temperature, and the radionuclide adsorption characteristics. Finally, thermal degradation of silicon layer above $1600^{\circ} \mathrm{C}$ has been reported with an increasing time-attemperature severity that is important to characterize as the primary TRISO barrier.

The resolution to this gap will be continuation of experiments and radionuclide transport model development to characterize the important complications of chemical reactions, sorption, and diffusive transport. Industry identified the transport of radionuclides in core materials as having a high priority. The effort is judged as high with a long-term timeline due to the dependence on experimental programs.

\begin{tabular}{|l|c|c|c|c|}
\hline Gap Resolution Action & Priority & Effort & Timeline & $\begin{array}{c}\text { NRC Vol3 } \\
\text { Crosscut }\end{array}$ \\
\hline $\begin{array}{l}\text { Research and model development on the transport } \\
\text { of radionuclides in core materials }\end{array}$ & High & High & $\begin{array}{c}\text { Long- } \\
\text { term }\end{array}$ & HTGR-M2.1 \\
\hline
\end{tabular}

\section{A.3.3 Graphite oxidation}

\section{Investigate graphite oxidation and irradiation characteristics; develop and validate models:}

The importance of oxidizing conditions has been identified as an important mechanism to enhance radionuclide release in graphite HTGR cores. Ref [33] states transients with air or steam oxidation "can lead to extensive degradation of the core components, including the fuel elements, and to a significantly enhanced release of radionuclides. The oxidation of graphite components, other carbonaceous materials and the $\mathrm{SiC}$ carbide layer in the fuel particles can play an important role in governing fuel performance. The oxidation of graphite is of primary importance with respect to the structural integrity of the graphite."

As reactor grade graphite materials and fuel compacts are improved, their oxidation characteristics following irradiation are important to quantify. The resolution to this gap is continued experimental studies on graphite oxidation from air and steam sources. These efforts will be used to predict the impact of these possible oxidizing sources during transient conditions. The data and analysis allow for development and benchmarking of models used to predict the progression of postulated transients with air and water ingression, which are among the key LBEs for HTGRs. 


\begin{tabular}{|l|c|c|c|c|}
\hline Gap Resolution Action & Priority & Effort & Timeline & $\begin{array}{c}\text { NRC Vol3 } \\
\text { Crosscut }\end{array}$ \\
\hline $\begin{array}{l}\text { Investigate graphite oxidation and irradiation } \\
\text { characteristics; develop and validate models }\end{array}$ & High & High & $\begin{array}{c}\text { Long- } \\
\text { term }\end{array}$ & HTGR-M2.2 \\
\hline
\end{tabular}

\section{A.4Circulating Inventory}

Despite having a chemically inert coolant, radionuclides may still transport within the bulk flow of the primary circuit in HTGRs. Computational modeling and experimental studies of radionuclide plating in the primary coolant loop are needed to quantify this behavior. Due to the ease with which some radionuclides can adsorb into graphite, the transport of radionuclidecontaining graphite dust throughout the reactor, as well as the deposition is important phenomena to the source term. The resolution is experimental studies and models to better characterize the graphite absorption of radionuclides, the deposition, and the liftoff in depressurization scenarios (see Section A.5.2).

\section{A.4.1 Thermal fluids experiments and models}

\section{Complete thermal fluids experiments; develop and validate models:}

Following their release from the fuel compacts into the gas stream, radionuclides transport through the primary system to leakage pathways into the reactor building. The associated aerosol and radionuclide gas behavior includes sorption of metallic radionuclides into graphic dust, condensation of radionuclide gas vapors onto cooler structures, agglomeration of aerosols, and aerosol deposition onto surfaces. Under transient conditions (e.g., a depressurization transient), previously deposited aerosols can liftoff back into the gas stream or wash-off with draining condensate films. The coupled thermal fluids and aerosol behavior and associated surface response drives radionuclide transport to the reactor building and environment. Continued development of experimental data quantifying the physical processes expected in HTGR operation and transient conditions is a critical part of the source term (i.e., how the radionuclides transport to the environment).

The resolution recommends coupled thermal fluids experiments and associated model development and code benchmarks with experimental data. The effort will improve prediction of the important processes of transport and deposition of radionuclides through the primary circuit, as well as the modeling of the liftoff and wash-off.

The NGNP PIRT [8] identifies circulating radionuclide behavior within a high-temperature graphite reactor as an important issue. The radionuclide adsorption and release from graphite dust and structures are identified as phenomena important to the source term. Similarly, Xu et al. identified the complexities of tritium transport adsorption into graphite and permutation through steam generator tubes [28]. The continuation of the HTGR thermal fluids experiments is identified as a medium priority and a long-term activity but high effort due to the cost of experimental programs. The development of models and benchmark data is also medium priority and long-term since it is dependent on the experimental results. The data benchmark and model development aspect of this resolution is expected to be a low level of effort relative to the experimental aspect. 


\begin{tabular}{|l|c|c|c|c|}
\hline Gap Resolution Action & Priority & Effort & Timeline & $\begin{array}{c}\text { NRC Vol3 } \\
\text { Crosscut }\end{array}$ \\
\hline $\begin{array}{l}\text { Complete thermal fluids experiments; develop } \\
\text { and validate models }\end{array}$ & Med & High & $\begin{array}{c}\text { Long- } \\
\text { term }\end{array}$ & HTGR-M2.1 \\
\hline
\end{tabular}

\section{A.4.2 Graphite dust transport and sorption}

\section{Conduct modeling and experimentation which elucidates graphite dust formation,} transport, and radionuclide sorption phenomenology:

During normal operations, high velocity helium circulates through the reactor core causing the formation of graphite dust. The radionuclides released during normal operation can accumulate with the graphite dust that is generated and subsequently distributed in the reactor system. The circulating radionuclides and graphite dust subsequently deposit on surfaces in the primary system. The graphite dust is primarily generated due to abrasion during the circulation of the pebbles through the core and transport within the fuel pebble recirculation system and has been characterized from during the German AVR operation [34, 35]. The dust generation from high velocity gases adjacent to prismatic fuel compacts is less understood. Furthermore, development of improved reactor grade graphite and fuel compacts has not been characterized [8].

The graphite dust is also an important sorption media for released radionuclides. As described in Section A.3.2, the $\mathrm{Cs}, \mathrm{I}, \mathrm{Kr}$, Sr, and $\mathrm{Xe}$ radionuclides are particularly susceptible to sorption on graphite or carbon. The resolutions to this gap are experiments and models to quantify the formation of graphite dust and adsorption of radionuclides in HTGRs as a function of fuel matrix, core design, irradiation behavior, or other factors. The fundamental research supporting the mechanisms of dust generation is needed to complete the characterization of radionuclide transport described in Section A.4.1. The major research and development needs include generating adsorption isotherms for radionuclides that display an affinity for graphite dust, investigating the formation and properties of carbonaceous crust on the inside of high temperature reactor coolant pipes, and confirming the predominant source of dust as abrasion between fuel spheres and the fuel handling system [36].

Both refs [36] and the HTGR PIRT [8] identify radionuclide behavior with graphite dust as an important issue that needs further investigation, noting that if radioactive dust is released to the confinement, it is typically considered to be a major source term factor. A medium priority with near-term initiation was assigned based on these technical reviews and discussions with industry. The experimental component may require multi-year effort although complex experimentation may not be needed. Additionally, dust transport modeling may build off of similarities to aerosol transport research and radionuclide-specific adsorption models may build off of knowledge of graphite sorption. For these reasons, the effort is rated as medium.

\begin{tabular}{|l|c|c|c|c|}
\hline Gap Resolution Action & Priority & Effort & Timeline & $\begin{array}{c}\text { NRC Vol3 } \\
\text { Crosscut }\end{array}$ \\
\hline $\begin{array}{l}\text { Conduct modeling and experimentation which } \\
\text { elucidates graphite dust formation, transport, and } \\
\text { radionuclide sorption phenomenology }\end{array}$ & Med & Med & $\begin{array}{c}\text { Near- } \\
\text { term }\end{array}$ & HTGR-M2.1 \\
\hline
\end{tabular}




\section{A.5Accident Scenario Modeling}

The licensing of HTGRs will be dependent on their performance in LBE scenarios. The consequences from these scenarios will be compared to safety metrics such as the LMP frequency-consequence target goals [37]. Previously identified challenges to HTGRs include air and water ingression, depressurization scenarios, and fuel matrix damages from rapid neutronic or thermal transients. Previous studies of HTGR risks have identified air and water ingression as important events causing graphite oxidation and challenges to fuel compacts $[8$, 33]. Another challenge is a depressurization transient that reduces the core heat removal effectiveness and opens a release pathway. Finally, rapid power and thermal transients may challenge the graphite compact integrity. The degradation of the fuel matrix holding the TRISO particles during rapid neutronic and thermal challenges could impact the radionuclide release, the matrix geometry, the heat removal requirements, and the number of TRISO failures. MST scenario modeling with uncertainty is needed to provide confidence in the source term in LBEs.

\section{A.5.1 Air and water ingress}

The ongoing and recommended experiment work in the other sections for advanced TRISOs discusses research priorities for radionuclide release behavior and quantification of TRISO failure characteristics (e.g., as a function of temperature for source term codes). This research and data form the basis and validation of the TRISO models that are used to assess the magnitude of the source term. Transient progression calculations connect the TRISO behavior models and research to the magnitude of the source term for regulatory acceptance. Air and water ingression are two postulated events that could increase the severity and magnitude of the source term [8]. Air ingression may occur following a primary system piping failure. The magnitude of the air ingression, the flow patterns into the reactor, and the impact of the oxidation on the magnitude of the source term are important to quantify. For example, high temperature graphite oxidation creates byproduct gases that may contribute to expelling radionuclides from the vessel, which may combust in the confinement. Severe oxidation of pebbles or the prismatic fuel compacts in the core may increase the fuel temperature and fail the TRISO fuel. Similarly, a tube rupture in an HTGR with a steam generator may introduce a water source that evaporates and oxidizes in the core. Steam oxidation with graphite has the additional adverse effect of creating hydrogen that could combust in the confinement.

The resolution of this gap is scenario identification and detailed models capable of assessing the thermal fluids flow patterns, the air and steam oxidation reactions, the formation and transport of the combustion gas by-products, and ex-vessel carbon-monoxide and hydrogen combustion models. Source term codes like MELCOR would benefit from benchmarks to detailed multi-dimensional code calculations.

\section{A.5.2 Depressurization}

In addition to air and steam oxidation, the depressurization of the primary system due to a pipe break is another important postulated event that could increase the severity and magnitude of the source term. During normal operations, high pressure helium flowing past the fuel compacts removes heat from the fuel. Following a depressurization transient, the convective 
heat transfer from fuel is severely diminished. The fuel heat-up and associated air ingression may lead to elevated temperatures and TRISO failures. In addition, the high in-vessel high depressurization flow may resuspend previous settled graphite dust and absorbed radionuclides. The reactor depressurization of high temperature helium into the confinement also challenges the ex-vessel pressure management features.

The resolution of this gap is scenario identification and detailed models and computer models capable of assessing the thermal fluids flow patterns, blowdown dynamics, the air and steam oxidation reactions, the fuel heat-up, and the fluid dynamic and structural response of the confinement structures, systems, and components (SSCs).

\section{A.5.3 Fuel matrix damage}

Finally, rapid power and thermal transients may challenge the graphite compact integrity. The role of irradiation on the structural integrity of the TRISO and the pebble or prismatic fuel compacts during these energetic events needs further investigation (e.g., see graphite irradiation discussion in ref [8].The resolution of this gap requires scenario identification and comparison of the thermal and mechanical stresses to irradiated graphite fuel and structures. 


\section{B Molten Salt Reactors}

\section{B.1 Core Neutronics and Fuel Depletion (Salt-fueled MSR only)}

There have been many efforts in modeling core neutronics and fuel depletion for salt-fueled MSRs but gaps still exist regarding:

- The integration of reactor physics models, depletion solvers, and on-line fuel processing for some standard geometries of circulating fuel geometries, as well as noncirculating MSRs

- The impact of delayed neutrons outside the core region

- The ability to conduct uncertainty/sensitivity analyses

- The level of validation against experimental data

- Benchmarking against high-fidelity neutron transport codes

- The activation of new materials being proposed for use in some MSRs

\section{B.1.1 Depletion Models}

Provide models for various generic core geometries for thermal and fast spectrum MSR concepts in NRC-supported code suites such as SCALE:

Generalizable fuel depletion models for standard geometries of various proposed types of MSRs would aid in the determination of the radionuclide inventory created within the reactor. This would entail integrating reactor physics models of the core to account for fuel depletion of the fuel salt, activation of fuel salt and core materials, decay of all radionuclides created, and on-line processing of the fuel salt when applicable. An example of MSR-specific fuel depletion and activation analyses would be the removal of a noble gas fission product from the liquid salt before it can be transmuted. This could allow for potentially large variations in some aspects of the radionuclide inventory between circulating fuel reactors and solid-fueled reactors. Also, it is unclear whether delayed neutron precursors will have a non-negligible impact on fuel depletion for fissions or activations that may occur outside the core. Additionally, the ability to conduct uncertainty or sensitivity analyses in the code may assist source term analysts in accident scenario analyses. Finally, it is recommended that deterministic or simplified neutron transport codes be benchmarked against higher fidelity codes.

Vendor discussions has generally indicated that this is a medium priority need. Although radionuclide inventories may be close to those found for LWRs or those found with existing tools such as SCALE/ORIGEN, some MSR designs may need a way to account for on-line fuel processing and its effect on the circulating source term, as well as the differences in inventories that occur with reactors of varying power level, fuel utilization, neutron energy, and flux. Additionally, regulatory confidence in the tool is needed to ensure the vendor is not over- or underestimating the radionuclide inventory, and thus, the source term.

Effort in completing this task is rated as medium, although it is considered a very cross-cutting capability that is important to all aspects of MSR-specific safety and design analyses. Therefore, development has already started and is ongoing, and is recommended to continue until an MSR-specific fuel depletion tool is fully verified and validated. A series of 
generalizable depletion models such as those found for LWRs in SCALE/ORIGEN is recommended for MSR source term analyses. This aligns with tasks recommended in the NRC's vision and strategy for SCALE code development for MSR reactor physics [2].

\begin{tabular}{|l|c|c|c|c|}
\hline Gap Resolution Action & Priority & Effort & Timeline & $\begin{array}{c}\text { NRC Vol3 } \\
\text { Crosscut }\end{array}$ \\
\hline $\begin{array}{l}\text { Provide models for various generic core } \\
\text { geometries for thermal and fast spectrum MSR } \\
\text { concepts in NRC-supported code suites such as } \\
\text { SCALE. }\end{array}$ & Med & Med & $\begin{array}{c}\text { Long- } \\
\text { term }\end{array}$ & $\begin{array}{c}\text { MSR-A1 and } \\
\text { D4 }\end{array}$ \\
\hline
\end{tabular}

\section{B.1.2 Activation}

Modeling the activation of secondary salts and other components in a flux of neutrons such as various structural materials, moderator or control rod materials, as well as the halide or inert cover gas itself:

Some designs may consider materials not previously utilized in nuclear applications, therefore a neutron activation analysis of the materials that are in a corresponding flux of neutrons may be necessary to ensure all radionuclides are being accounted for in the inventory. Many neutron transport codes exist for neutron activation analyses, therefore only the development of models specific to the reactor system/materials remains to be completed by the analyst.

Vendor discussions indicate this is a low priority need, but activations of salt components, including the halide itself (e.g., ${ }^{35} \mathrm{Cl}$ ), as well as structural materials are important to consider when compiling total radionuclide inventories that need to be considered when proceeding with the ensuing steps of a source term analysis.

Effort in completing this task is rated as low as many radiation transport codes currently exist for this application (such as that found in SCALE), thus providing the analyst an existing tool to build a custom model to analyze its activation. Therefore, the only actionable item is to develop the reactor-specific models in the code for the irradiated material of interest in the corresponding neutron environment. It is recommended that existing radiation transport codes such as those found in the SCALE suite be used to calculate the activation product inventory in the relevant materials in the core and surrounding regions. If the activated products are stationary (e.g., activated structural component), then additional analyses will be needed to decide the likelihood it will be leached out of the alloy and into the salt (See Section B.6.1). Oppositely, such a corrosion modeling tool can first be used to assess the quantity of structural components that will leach out of the alloy elsewhere in the loop. These corrosion products will then flow with the salt through the core, thus becoming activated. In either case, the tools required to model neutron activation of materials exist and should be used to provide an activation product inventory as input to the source term modeling code (e.g., MELCOR). 


\begin{tabular}{|l|c|c|c|c|}
\hline Gap Resolution Action & Priority & Effort & Timeline & $\begin{array}{c}\text { NRC Vol3 } \\
\text { Crosscut }\end{array}$ \\
\hline $\begin{array}{l}\text { Modeling the activation of secondary salts and } \\
\text { other components in a flux of neutrons such as } \\
\text { various structural materials, moderator or control } \\
\text { rod materials, as well as the halide or inert cover } \\
\text { gas itself. }\end{array}$ & Low & Low & $\begin{array}{c}\text { Long- } \\
\text { term }\end{array}$ & \\
\hline
\end{tabular}

\section{B.2 Core Neutronics and Fuel Depletion (FHR only)}

See Section A.1

\section{B.3 TRISO Fuel Particle Releases (FHR only)}

See Section A.2

\section{B.4Salt Chemistry}

Much research and development in molten salt chemistry is recommended, especially related to the vapor pressures and chemical speciation of fission products as a function of composition, system temperature, and pressure. Computational modeling tools have been created and are being developed but the underlying thermodynamic data that the tools rely on is still incomplete. The thermodynamics of molten salt solutions are highly non-ideal and the energetic interactions between components in the salt create a highly coupled thermochemical system that can only be modeled with fidelity with experimentally derived thermodynamic data of the component interacting in a salt solution of other components. This underlying thermodynamic data still needs to be found for some salt-soluble fission products. Ultimately, the developed database and equilibrium solver will also need to be validated with experimental data such as vapor pressure data for all relevant components when dissolved in the salt system of interest. Additionally, a sensitivity study is recommended to assess the sensitivity of the modeling tool on this underlying data, as well as its associated uncertainties, on chemical speciation as well as transport phenomena such as vaporization, precipitation, or other figuresof-merit. Eventually, a radionuclide mass transport or species tracking tool should be developed which couples this thermodynamic database and modeling tool with other transport phenomena. Such phenomena might include bubble nucleation and growth, solid species precipitation or bulk flow, deposition, or other methods contributing to local concentration gradients of certain radionuclides. Finally, it is recommended that research programs in radiolytic effects of molten salts continue, specifically as it relates to radionuclide transport.

\section{B.4.1 Thermodynamic database}

Develop a thermodynamic database and GEM solver of the necessary thermochemical properties for molten fluoride and chloride salt systems, including relevant base salt components but also including fission products that may exist as soluble in the salt solution; Validate the database:

The ability to estimate vaporization of specific radionuclides is a main focus. Development of the Molten Salt Thermodynamic Database (MSTDB-TC) has started to address this gap but continued experimental work will be needed to add thermochemical data for more elements 
that exist in an MSR salt system such as fission products (e.g., iodine) or activated corrosion products (e.g., chromium). Because molten salts exhibit often highly non-ideal thermochemical behavior, this will need to be quantified somehow (e.g., activity coefficients) to accurately assess the level of radionuclide vaporization from the salt to the gas not only in accident scenarios, but also within the reactor vessel. For example, the vapor pressure of a pure component fluoride is not an accurate representation of how easily it will volatilize if it is dissolved (soluble) in a molten fluoride solution. Fission product solubility is also important as some elements may precipitate or form solid solutions at certain temperature ranges or compositions. Additionally, the impact of having dilute concentrations of fission products in the salt needs to be investigated to assess the validity of assuming non-ideal thermochemical behavior for the fission product. Finally, the database will need to be coupled to a Gibbs Energy Minimization (GEM) solver such as Thermochimica (Yellowjacket is also under development) to be used either standalone or within a multiphysics code for equilibrium calculations, and so confirmation of this development task will be needed.

Additionally, a major question with such a tool is how confidence in the model can be improved. Validation of the model and underlying thermochemical data is very important to ensure reliable simulation of fission product evaporation under various temperatures, pressures, and salt compositions. It is recommended that an experiment be designed to measure the vaporization of salt-soluble fission products from various salt systems to verify the thermochemical data used for the system components (base salt components and fission product solutes).

Vendor discussions have indicated this is a high priority need and one of the biggest concerns in estimating the transport behavior of radionuclides from the salt. There is a concern that vendor calculations may not match results from the tools or databases used by the regulator, therefore QA is a very important development task. One chemical species of considerable importance to MSR source term is cesium iodide and additional experimental work is needed to compile the necessary thermodynamic data that is provided as input to MSTDB to accurately model its volatilization behavior. An additional recommendation from discussions with vendors include improved usability (e.g., documentation) and accessibility for these tools, which should be addressed as the tools are developed.

Effort in completing this task is rated as high as the necessary work is both experimental and computational/analytical in nature, and both can be time consuming depending on the fission product investigated. There is a strong near-term need for this thermochemical modeling tool. Fortunately, work has already begun as part of NEAMS and other DOE-NE programs in developing tools (MSTDB and Thermochimica/Yellowjacket) which satisfies some near-term salt chemistry needs, but more work will be needed to add to the database and satisfy modeling needs for all other relevant elements (all salt-soluble radionuclides of interest) in the long-term. This task aligns with development recommendations for MELCOR, which is planning to implement the same database and GEM solver, MSTDB-TC and Thermochimica (or Yellowjacket), respectively.

As an example, the code workflow for coupling these thermodynamic tools with a code such as MELCOR is briefly described here. This will likely require an application programming interface. A code such as MELCOR will provide temperature, pressure, and elemental 
composition, while the thermodynamic database and GEM solver can provide the mass of stable phases, their chemical speciation, gaseous partial pressures, chemical potentials, heat capacity, or other related parameters.

\begin{tabular}{|l|c|c|c|c|}
\hline Gap Resolution Action & Priority & Effort & Timeline & $\begin{array}{c}\text { NRC Vol3 } \\
\text { Crosscut }\end{array}$ \\
\hline $\begin{array}{l}\text { Develop a thermodynamic database and GEM } \\
\text { solver of the necessary thermochemical properties } \\
\text { for molten fluoride and chloride salt systems, } \\
\text { including relevant base salt components but also } \\
\begin{array}{l}\text { including fission products that may exist as } \\
\text { soluble in the salt solution; Validate the database }\end{array}\end{array}$ & High & Med & $\begin{array}{c}\text { Near- } \\
\text { term }\end{array}$ & MSR-M3.2 \\
\hline
\end{tabular}

\section{B.4.2 Sensitivity analysis}

\section{Complete a sensitivity analysis to investigate how varying component thermodynamic} values alters computed chemical and phase equilibria, in addition to an uncertainty propagation analysis:

It is unclear the impact that each component thermodynamic value has on the estimation of chemical and phase equilibria from the tool developed in Section B.4.1, as the Gibbs energy minimization calculation and corresponding energetic interactions represented by

thermodynamic values are highly coupled via a complex solution model. For the application of radionuclide speciation in a generic MSR salt, it may be beneficial to complete a sensitivity study on the impact of various underlying thermodynamic data values, as well as their uncertainties, on the computed behavior of the system. This computer behavior may include figures-of-merit such as partial vapor pressures of certain important radionuclides, solubility and precipitation of others, or other metrics which influence transport. Ultimately, it is recommended to estimate the impact of these thermodynamic parameters, along with the effect of having a diverse composition, on other transport phenomena such as radionuclide entrainment, bubble transport, salt aerosolization, vaporization, precipitation, and deposition.

Vendor discussions have led to the conclusion that this is a high priority gap and may only require a low effort to complete. Additionally, the results of which may guide the development of other gap resolution tasks therefore it is classified as a near-term need. It will be important to establish the scope of study relevant to radionuclide transport as well as the thermodynamic parameters which are altered within their ranges of deviation and uncertainty.

\begin{tabular}{|l|c|c|c|c|}
\hline Gap Resolution Action & Priority & Effort & Timeline & $\begin{array}{c}\text { NRC Vol3 } \\
\text { Crosscut }\end{array}$ \\
\hline $\begin{array}{l}\text { Complete a sensitivity analysis to investigate how } \\
\text { varying component thermodynamic values alters } \\
\text { computed chemical and phase equilibria, in } \\
\text { addition to an uncertainty propagation analysis }\end{array}$ & High & Low & $\begin{array}{c}\text { Near- } \\
\text { term }\end{array}$ & \\
\hline
\end{tabular}




\section{B.4.3 Mass transport coupling}

\section{Develop radionuclide mass transport modeling tools which couple with the thermodynamic modeling tool:}

As fissions, neutron activation, and decays occur, the composition and redox potential of the salt changes. For more accurate radionuclide speciation and species tracking within the salt solution, a modeling tool will be needed which couples the aforementioned thermodynamic database and GEM solver with various liquid, gas, and solid phase phenomenology, including, but not limited to, bubble nucleation and growth, solid precipitate formation, insoluble precipitate flow, noble metal plateout at phase interfaces (e.g., bubble surface interactions), and any other phenomena which may create local concentration gradients as a function of composition and temperature. Insights can not only be found from higher fidelity thermal fluids tools (e.g., CFD models) such as those designed for bubble transport or two-phase flow dynamics (See Section B.5), but also insights can be found from reduced order multiphysics and engineering scale tools (e.g., the NEAMS tool Mole) which may aim to couple multiple phenomena together to better understand the complex multiphysics of species transport within the primary loop of MSRs. Similar models are expected to be implemented into the MSM module of MELCOR to aid in severe accident analysis of MSRs.

Vendor discussions have indicated this to be a high priority resolution as there are many questions regarding species transport within the flowing salt, especially as a function of the constantly changing salt composition and temperature gradients. Although, it is unclear if the impact of these phenomena on source term modeling warrants a near-term development timeline, therefore this is left as a long-term development objective. The biggest salt chemistry source term concerns such as fission product vaporization and actinide solubility can be resolved temporarily with the tools developed in Section B.4.1 without needing to couple to mechanical and fluid behavior models. The coupling of salt chemistry effects on species tracking are recommended for eventual development as development of the individual models is completed. The development of such a tool is expected to have a high degree of effort as it is expected to require a multi-year effort for model and code development. Fortunately, some of these developed models are already planned in the NEAMS code Mole, as well as inclusion in the MSM module within MELCOR.

\begin{tabular}{|l|c|c|c|c|}
\hline Gap Resolution Action & Priority & Effort & Timeline & $\begin{array}{c}\text { NRC Vol3 } \\
\text { Crosscut }\end{array}$ \\
\hline $\begin{array}{l}\text { Develop radionuclide mass transport modeling } \\
\text { tools which couple with the thermodynamic } \\
\text { modeling tool }\end{array}$ & High & High & $\begin{array}{c}\text { Long- } \\
\text { term }\end{array}$ & MSR-M3.2 \\
\hline
\end{tabular}

\section{B.4.4 Radiolytic effects}

\section{Complete studies on the effect of radiation on salt chemistry:}

Very little has been published on the effect of radiation on salt chemistry phenomena. Therefore, it is important to explore the extent to which radiolytic effects have on chemical speciation, phase changes, and other reactions with radicals that may be formed in the salt as a result of radiation. For example, during an inspection of the decommissioned MSRE fuel and flush salts 25 years after shutdown, it was noticed that considerable fluorine gas, and, as a 
result, volatile UF6, was found in the gas space above the cooled salt [22]. The increased creation of fluorine radicals as the irradiated salt cools should be explored, although this corresponds more so to salt chemistry analyses for salt spills and decommissioned reactors, as opposed to normal reactor operation (See Section B.10).

Vendor discussions indicate this to be a medium priority need for source term modeling, as halogen radical formation seems to occur dominantly as the salt cools, but there are potentially other unknowns associated with salt radiolytic effects. Because a considerable experimental component is assumed including irradiation of samples, the level of effort required is deemed to be high. Fortunately, work is ongoing on irradiation of certain molten salt systems as a part of other DOE research programs, and so there may be cross-cutting research to propose. As radiolytic effects on salt chemistry and radionuclide transport impacts not just source term, but also reactor operation and fuel qualification, there may be information learned from other research programs over the long-term timeline which is recommended for this development objective.

\begin{tabular}{|l|c|c|c|c|}
\hline Gap Resolution Action & Priority & Effort & Timeline & $\begin{array}{c}\text { NRC Vol3 } \\
\text { Crosscut }\end{array}$ \\
\hline $\begin{array}{l}\text { Complete studies on the effect of radiation on salt } \\
\text { chemistry }\end{array}$ & Med & High & $\begin{array}{c}\text { Long- } \\
\text { term }\end{array}$ & \\
\hline
\end{tabular}

\section{B.5 Bubble Transport}

Experimental work on modeling bubble transport in molten salt reactor systems has been completed for some gases in some systems, including noble gases and some volatile fluorides. A deeper understanding of the phenomenon as a function of salt composition and gas type is lacking, warranting additional experimental work to elucidate bubble transport properties. Some numerical models have been developed for noble gas migration in MSRs, but validation of these models is needed, while there may also be a gap in modeling other types of volatiles if different from noble gases. Radionuclide entrainment in the bubbles needs to be investigated and compared to that seen with liquid sodium pools for SFRs. Additionally, bubble or gas transport into graphite or other porous materials should be investigated more in order to accurately model this phenomenon in MSRs.

\section{B.5.1 Gas solubility and radionuclide entrainment}

Conduct experimental studies on the solubility, bubble agglomeration, and transport of noble gases, volatile fluorides, and volatile noble metals through molten salts, as well as corresponding radionuclide entrainment; Develop and validate models:

It is recommended to determine the solubility constants of noble gases in molten salts to understand how these fission products (e.g., isotopes of $\mathrm{Xe}$ and $\mathrm{Kr}$ ) transport through the salt and eventually leave the liquid solution. It is believed many salt-soluble fission products may also end up adsorbed onto graphite (or similar materials) due to the mobility of their noble gas precursors. Noble gas solubility in molten salts therefore has considerable importance to source term modeling beyond just the anticipated transport of the source term to the off-gas system. But the mobility of noble gases through graphite or similar porous materials also should be investigated and quantified to effectively track the source term mechanistically. This 
phenomenological area also includes the removal (via bubbling) of volatile metal fluorides/chlorides, other metal halides which may be present in the system (iodides, bromides, etc.), hydrogen/tritium fluoride, as well as carbon fluorides which may exist in some graphitecontaining systems. Also, some reactor designs may utilize a fueled molten salt which isn't circulating; therefore, experiments must also consider bubble transport without fluid flow (i.e., stationary fuel salt without sparged gas flow).

Additionally, it will be important to quantify the amount of radionuclides which will transport out of the salt and become entrained in or on bubbles flowing through the liquid solution. This includes understanding the underlying theory of this process, as well as developing coefficients to quantify the level of entrainment in bubbles as a function of flow rate, flow type, gas type, and even radionuclide chemical group or class. An important mechanism is the rupture of gas bubbles at the salt surface, which, for example, leads to increased mobility of some noble metals which might adhere to the bubble surface.

Experiments should be carried out to elucidate additional phenomena related to the way noble metals transport and behave in MSRs. It is believed that their behavior is highly dependent on their redox state, and thus the redox potential of the system. This includes experiments on the electrochemical properties of this class of elements, in addition to other studies which may elucidate the physicochemical properties of these systems. For example, noble metals may volatilize easily, form mists or aerosols easily (See Section B.7) or create a foam layer at the gas-liquid interface in the reactor. Experimental salt loops may be useful, which can offer insight into the flow of noble metals at the gas-liquid interface (or on the surface of bubbles). It has been postulated that noble metals act as surfactants, affecting bubble formation and transport dynamics [38]. Noble metals represent a considerable amount of radioactivity and there is no consensus understanding on how they behave as a class within the reactor. This includes some combination of: salt soluble fluorides which stay in the liquid solution or exit the solution as a mist; volatile fluorides vaporizing from the solution; metals flowing at the gas-liquid or bubble interface; and metals depositing on alloys or graphite (See Section B.6).

Computational models of bubble transport (such as those designed in CFD codes) should be further developed and catered to source term modeling applications and should be validated to the aforementioned experimental data that is collected. These higher fidelity models can also be used to benchmark lower fidelity or reduced order models which may be used as a less computationally expensive alternative in integral analysis or systems analysis codes such as MELCOR. Two-phase flow or insoluble species flow can also be modeled with such codes.

Vendor discussions have indicated this to be a high priority. The importance of bubble transport to radionuclide transport in molten salt systems is perceived to be high. Some experimental work in the past has provided a baseline understanding of how noble gases dissolve and diffuse through salts as a function of temperature and salt composition. But is not clear the level to which radionuclide entrainment in bubbles occurs, and if data from systems with other liquids can be used (e.g., aqueous or liquid sodium). This could have a significant impact on the transport of certain fission products through the salt to the off-gas system. More experimental work may be needed to validate models that have been created, including the CFD work that is ongoing by many fluid dynamics modelers. 
Because filling some of these information gaps requires experimentation, often with custom apparatuses, the effort required is expected to be high. Fortunately, there is some existing data on noble gas solubility and some CFD models have been made on this topic. Additionally, there may be some cross-cutting efforts with bubble transport in the sodium pool of SFRs [2, 39], and so these assumptions might be sufficient for preliminary source term calculations. Nonetheless, there is an urgency in developing an understanding of how bubbles transport in molten salts, as well as how radionuclides entrain within or on the surface of bubbles, therefore some near-term tasks are recommended as part of this gap resolution. But completion of the resolution can occur on a longer timeline as soon as the most critical information relevant to radionuclide transport is found.

This also overlaps with that recommended by the NRC's modeling and simulation code development strategy as part of the proposed development of the Molten Salt Model (MSM) within MELCOR [2, 39].

\begin{tabular}{|l|c|c|c|c|}
\hline Gap Resolution Action & Priority & Effort & Timeline & $\begin{array}{c}\text { NRC Vol3 } \\
\text { Crosscut }\end{array}$ \\
\hline $\begin{array}{l}\text { Conduct experimental studies on the solubility, } \\
\text { bubble agglomeration, and transport of noble } \\
\text { gases and volatile fluorides through molten salts, } \\
\text { as well as corresponding radionuclide } \\
\text { entrainment; Develop and validate models }\end{array}$ & High & High & $\begin{array}{c}\text { Near- } \\
\text { term }\end{array}$ & MSR-M3.2 \\
\hline
\end{tabular}

\section{B.6Radionuclide Deposition}

The deposition of radionuclides inside MSR systems is very important when tracking the source term. Deposition can occur electrochemically and physically onto various material types including structural alloys, heat exchanger materials, and graphite or similar porous solid materials, such as those which might be used as a moderator or control rod. There has been much experimental research in corrosion of alloys in molten salts, but additional work is recommended in modeling electrochemical behavior, which includes both corrosion or leaching from the alloy and deposition onto the alloy. A deeper understanding of the chemistry that controls the underlying theory would aid in understanding how some of these fission product elements and salt systems interact with certain structural materials. A more complete understanding of the electrochemistry of fission products (e.g., noble metals) and activated corrosion products will assist in validating time-dependent models for electrochemical reactions which have been coupled with flow modeling. Further work is needed to elucidate additional phenomena and confirm hypotheses related to the migration of noble metals which appears to be a function of the element's redox state. Finally, the adsorption, diffusion, retention, and release of radionuclides from graphite is important to understand (when applicable). 


\section{B.6.1 Alloy corrosion and deposition}

\section{Complete corrosion and deposition experiments with relevant alloys and various fission product-containing salt systems:}

It is recommended to complete static and dynamic experiments (as needed) on the corrosion of various prototypical structural materials with relevant chloride and fluoride salt systems, adding realistic concentrations of potential impurities and fission products. This provides an understanding of the concentration of corrosion products that can be leached from the alloy into the salt. Many of these elements can be activated if they stay in the salt and enter a flux of neutrons. It is also important to understand the electrochemical deposition behavior not only for these corrosion products, but also for fission products, such as the noble metals. Adding various noble metals to the experimental salt may provide better understanding on the impact of these fission products on corrosion/deposition to the alloy being tested, as some may have a protective effect to the alloy while also reducing the circulating source term.

Vendor discussions have indicated this to be a medium priority for source term modeling. While it is very important to MSR materials design, it has a medium impact on the source term. Experiments related to electrochemical behavior is important to understanding the mobility of various elements which may change redox state easily. Therefore, completing experiments on the electrochemical properties of various relevant radionuclides in representative salt systems and alloys of interest is recommended. These experiments can often be time consuming, therefore the effort required is rated as high. Accurate modeling of the redox potential of the system (and thus the salt chemistry) will rely on this information, but it is not urgent, therefore this has a long-term timeline. When modeling the source term release associated with the salt or vapor escaping the reactor system, it would be conservative to assume no radionuclides are deposited within the primary loop and are retained in the salt or vapor phases.

\begin{tabular}{|l|c|c|c|c|}
\hline Gap Resolution Action & Priority & Effort & Timeline & $\begin{array}{c}\text { NRC Vol3 } \\
\text { Crosscut }\end{array}$ \\
\hline $\begin{array}{l}\text { Complete corrosion and deposition experiments } \\
\text { with relevant alloys and various fission product- } \\
\text { containing salt systems; Develop and validate } \\
\text { models }\end{array}$ & Med & Med & $\begin{array}{c}\text { Long- } \\
\text { term }\end{array}$ & \\
\hline
\end{tabular}

\section{B.6.2 Graphite adsorption and diffusion}

Complete experimental studies on the adsorption of radionuclides onto graphite, and the eventual diffusion into and retention within the porous material; Develop and validate models:

If a system contains graphite or other porous solid materials, radionuclides may adsorb onto the solid and diffuse into the material over time with a dependence on temperature. Alternatively, some noble gases may easily diffuse into the solid and decay to a daughter product which is less mobile. Through whatever method, radionuclides may be retained in the porous material yet may be released as a function of temperature, such as during a temperature excursion during an accident. Therefore, the release of certain radionuclides from the material which may occur given a high enough temperature must be accounted for with experimental efforts and modeling tools. 
Vendor discussions have indicated this to be a medium priority as not all reactor designs contain graphite moderator or similarly porous material which will retain radionuclides. Relevant experiments will demand a high amount of effort given the large number of radionuclides which should be tested, although there may be some literature on this topic which should be reviewed to reduce effort. A long-term timeline for completion is designated given the medium priority and the potential information which may already exist in literature, sufficient for initial source term modeling efforts.

\begin{tabular}{|l|c|c|c|c|}
\hline Gap Resolution Action & Priority & Effort & Timeline & $\begin{array}{c}\text { NRC Vol3 } \\
\text { Crosscut }\end{array}$ \\
\hline $\begin{array}{l}\text { Complete experimental studies on the adsorption } \\
\text { of radionuclides onto graphite, and the eventual } \\
\text { diffusion into and retention within the porous } \\
\text { material; Develop and validate models }\end{array}$ & Med & Med & $\begin{array}{c}\text { Long- } \\
\text { term }\end{array}$ & \\
\hline
\end{tabular}

\section{B.6.3 Aerosol and gas phase deposition}

Radionuclides can mechanically deposit on solid surfaces after being transported as aerosols. See Section B.7 for recommendations on resolving information gaps regarding aerosols.

\section{B.7Aerosol Formation and Transport}

Aerosol modeling of molten salts is relatively immature, except for some experimental studies in temperature-induced salt aerosol formation and supercooling, as well as evidence of salt mists formed during operation of MSRE. Fortunately, there is a large degree of existing knowledge regarding mechanically generated aerosols of more common liquids (e.g., aqueous solutions), which is potentially applicable to molten salts. But it is presently unknown whether these models and correlations can be extrapolated to represent molten salt solutions. For example, molten salts have a higher surface tension than aqueous solutions, and the effect of temperature and composition on surface tension, and thus aerosol formation, is still an information gap. A study to determine the level of applicability of existing aerosol knowledge based on experimental efforts involving molten salts is recommended. These experiments should be catered to elucidating both aerosol formation and aerosol transport phenomena. An example of information that should be found from experimental efforts is the size distribution of aerosolized particles, or specifically the threshold particle diameter for droplet formation. Ultimately, modifications to existing aerosol models in severe accident analysis codes can be made (if needed).

\section{B.7.1 Formation}

Conduct experimental studies on mechanically induced or thermally induced molten salt aerosol formation, Develop and validate models:

In order to understand differences between high-temperature salt aerosols and lower temperature aqueous aerosols, it is necessary to complete experimental aerosol studies at varying scales and sizes in order to quantify resulting mechanically induced aerosol formation, as well as temperature-induced aerosol formation (e.g., evaporation followed by condensation). 
Especially important will be investigating aerosolization that occurs via potential two-phase flow of a salt (e.g., FLiBe) and gases, such as that which may occur in salt sprays which release gases to the off-gas system. Understanding the ease with which aerosols are formed via sprays is especially important as this was used in the form of a salt-spraying pump bowl during MSRE to aid in the removal of volatile fission products from the circulating fuel salt. The addition of fission product surrogates can help elucidate speciation-related phenomena between the liquid salt, aerosol salt, and gas phases. Additionally, this is important in the event of a breach in SSCs which may result in salt leaking at high enough velocity or large enough temperature difference to induce aerosol formation.

Adding complexity to the formation of aerosols in MSR systems is the effect of noble metals which may act as surfactants on bubble surfaces depending on the element, its redox state, and the formation of bubbles. Aerosols formed via bubbles bursting at the salt-gas interface is a phenomenon of interest, and the resulting effect of noble metals on bubble transport, foam formation, and aerosol formation should be explored (See Section B.5).

Vendor discussions have indicated this to be a high priority. The difficulty in accurately modeling two-phase flow has been noted and the use of spray bowls within the reactor systems is desirable. Additionally, it is still unclear the level to which noble gases volatilize, but it may be possible that these often-volatile elements enter the gas space as salt-dissolved aerosols as well as vaporized species.

The effort is rated as high due to the high degree of customized experimental set-ups and planning. Some large-scale experiments will be necessary to fully understand some of the ways aerosols can be formed in both normal operation within the reactor as well as during severe accident scenarios such as an energetic splashing of salt during a spill. Some information gaps will be resolved from the currently ongoing Salt Spill experiments taking place at Argonne and supported by the MSR Campaign [21], but more experimentation will ultimately need to occur to get the current state of knowledge of molten salt aerosols to that of aqueous aerosols. Additionally, these experiments can be designed to resolve some of the other aerosol information gaps, such as transport and removal mechanisms (See Section B.7.2).

Because this is a relatively unknown phenomenon with high regulatory impact, the results of this are deemed to be urgent and therefore this has a near-term timeline for completion. As results are found from experiments, models should be developed and validated to properly account for aerosol formation within codes such as MELCOR or others. Additionally, the applicability of existing aqueous aerosol models can be assessed (See Section B.7.3). This resolution is also included as part of the development tasks for the Molten Salt Model (MSM) within MELCOR as part of the NRC's code development vision and strategy [2].

\begin{tabular}{|l|c|c|c|c|}
\hline Gap Resolution Action & Priority & Effort & Timeline & $\begin{array}{c}\text { NRC Vol3 } \\
\text { Crosscut }\end{array}$ \\
\hline $\begin{array}{l}\text { Conduct experimental studies on mechanically } \\
\text { induced or thermally induced molten salt aerosol } \\
\text { formation; Develop and validate models }\end{array}$ & High & High & $\begin{array}{c}\text { Near- } \\
\text { term }\end{array}$ & MSR-M3.2 \\
\hline
\end{tabular}




\section{B.7.2 Transport and removal}

\section{Conduct experimental studies on salt aerosol transport and removal mechanisms; Develop and validate models:}

Understanding how molten salt aerosols transport in a volume of gas will be very important to understanding source term releases in a severe accident in addition to transport inside a reactor vessel or pump bowl during normal operation. There is much operational evidence from MSRE that indicates salt mists or aerosols were formed in the pump bowl which sprayed salt down into a pool of salt, but operators had difficulty in understanding how it transported within the headspace and attempts at shielding its transport to off-gas lines were often unsuccessful. Experimental studies to elucidate aerosol formation should be coupled to experiments which track or quantify transport properties of the formed aerosol. The properties can be correlated to parameters describing the environment, such as head space volume, gas type, flow properties, and filtration. Note if the shape factors are different from aqueous aerosols and develop new models and modeling tools if so.

After it is understood how a salt aerosol forms and transports, it will be important to be able to model its decontamination or removal. It is likely the same methods and models for aerosols of other types will be applicable but experiments still need to be carried out to confirm this. For example, it is important to estimate the particle size threshold between salt aerosols and salt droplets (e.g., 50 microns for water) to determine when salt aerosols may "drop out" from the gas space. It is very important to understand the particle size distribution of aerosols relative to the size threshold for respirable aerosol particles, as respirable particles carry a larger weight in dose consequence models. In addition to aerosol particle size distributions, it is also important to develop an understanding of the chemical composition of aerosol particles, as most particles will be agglomerations of different radionuclides in varying concentrations, adding another layer of complexity to aerosol source term modeling. Finally, there is also questions as to whether the same spray models and correlations for removal coefficients will apply for salt aerosols as they have been developed for aqueous aerosols, so developing the experiments to elucidate this is important.

Vendor discussions have indicated this to be a high priority due to the unknowns surrounding particle size threshold as well as the question surrounding whether the aqueous aerosol transport models can be extrapolated based on material property differences. In order to assess the applicability of aqueous aerosol models, experimental work should be completed with molten salt aerosols. These experiments may require considerable planning and materials although often it can be done in conjunction with the aerosol formation experiments. Some information gaps will be resolved from the currently ongoing Salt Spill experiments taking place at Argonne and supported by the MSR Campaign [21], but more experimentation will ultimately need to occur to get the current state of knowledge of molten salt aerosols to that of aqueous aerosols. The full scope of aerosol transport experiments and analysis should warrant a high degree of effort. Nonetheless, the urgency for this information demands a near-term timeline.

As data is collected, models and correlations should be developed and validated to properly account for aerosol transport and removal phenomena within codes such as MELCOR or others. An example of input MELCOR may need to model aerosol transport would be the 
particle size distribution of the formed aerosols. This distribution can be elucidated through experiments, often the same experiments proposed in the preceding section on aerosol formation. Similarly, as experiments are executed, the applicability of existing aqueous aerosol models can be assessed (See Section B.7.3). Finally, this resolution is also included as part of the development tasks for the Molten Salt Model (MSM) within MELCOR as part of the NRC's code development vision and strategy [2].

\begin{tabular}{|l|c|c|c|c|}
\hline Gap Resolution Action & Priority & Effort & Timeline & $\begin{array}{c}\text { NRC Vol3 } \\
\text { Crosscut }\end{array}$ \\
\hline $\begin{array}{l}\text { Conduct experimental studies on salt aerosol } \\
\text { transport and removal mechanisms; Develop and } \\
\text { validate models }\end{array}$ & High & High & $\begin{array}{c}\text { Near- } \\
\text { term }\end{array}$ & MSR-M3.2 \\
\hline
\end{tabular}

\section{B.7.3 Applicability of existing aerosol models}

Compare experimental molten salt aerosol data to that of aqueous or sodium aerosols: After experiments are completed, it will be desirable to assess the applicability of existing aerosol modeling tools and knowledge to reduce model and code development activities where possible. Some aerosol formation, transport, and removal models and correlations may be applicable between the more common aerosol types (e.g., aqueous) and molten salt aerosols or "mists".

Vendor discussions have indicated this to be a high priority, similar to the experimental efforts on aerosol modeling. But this assessment creates the potential for vendors to tap into the wide range of information published on aqueous aerosols and utilize existing models and codes to model this very important phenomenon. The effort required is rated as low because it should not require significant additional effort to compare experimental molten salt aerosol data to corresponding data for other aerosol types. This should require analyzing the experimental data and developing the same correlation types and coefficients which can be used as input to the same codes or models previously developed for LWR aerosol modeling. Because this follows alongside the same timeline and importance as the experimental aerosol efforts, this has a nearterm timeline, but cannot be resolved until more experimental data on molten salt aerosols is obtained. This resolution is also recommended as part of the NRC's vision and strategy in terms of developing models for molten salt aerosol phenomena within MELCOR [2].

\begin{tabular}{|l|c|c|c|c|}
\hline Gap Resolution Action & Priority & Effort & Timeline & $\begin{array}{c}\text { NRC Vol3 } \\
\text { Crosscut }\end{array}$ \\
\hline $\begin{array}{l}\text { Compare experimental molten salt aerosol data to } \\
\text { that of aqueous or sodium aerosols }\end{array}$ & High & Low & $\begin{array}{c}\text { Near- } \\
\text { term }\end{array}$ & MSR-M3.2 \\
\hline
\end{tabular}

\section{B.8Tritium Modeling}

Tritium transport modeling in MSR or FHR systems is relatively mature compared to other source term modeling areas, yet some development tasks are still recommended, as well as validation of the tools with experimental data. Because tritium gas diffuses with relative ease through many materials, there is a question as to how it is best modeled from a source term perspective. For example, tritium may be modeled as an effluent during the normal operation of a reactor, in which case there may be standalone codes which can do this with relatively 
little modification to cater to the specific reactor design and materials. These codes have been described in previous reports [5, 40]. It may also be relevant to model the single release of a larger source of tritium during a transient scenario, where temperature transients or even accident scenarios may allow for it. This sudden release of a potentially larger amount of tritium is probably best modeled within severe accident or systems analysis codes which can account for multiphysics aspects through integral analyses. In order to achieve this, the relevant tritium transport models within standalone codes (or recently summarized in ref [41]) need to be coupled and/or integrated into such codes. These include codes with models which account for sorption, diffusion, and other modes of transport. For example, work is ongoing to incorporate relevant tritium transport phenomena into the SAM code under NEAMS [40].

Additionally, because tritium plays a large role in salt chemistry, and thus speciation, of MSRs, it may be advisable that thermochemical modeling tools and tritium formation, transport, and release models be coupled together. One example would be to incorporate thermochemistry phenomena (MSTDB \& Yellowjacket [15]) into a systems analysis code such as SAM, where tritium sinks and sources can be accounted for at every time step along with chemical reactions involving tritium and other radionuclides in the system. This may be important for some systems as tritium can have a considerable impact on the redox potential of the system, which affects radionuclide transport, corrosion, and actinide oxidation states.

Finally, one concern is the need for more experimental data concerning tritium interactions with various materials, especially graphite and alloys. It is recommended that existing values for diffusivity of tritium through these common MSR materials be validated. Similar experiments may be advisable as new and different materials are proposed for a specific design, especially if proposed as a specific tritium trapping material to actually contain and trap the tritium source term. A review is recommended regarding the release of tritium from graphite during normal operation, as well as during an accident scenario where there may be a temperature excursion or a depressurization.

\section{B.8.1 Releases during transients}

\section{Evaluate the impact of tritium releases during transient scenarios with sensitivity studies and inventory calculations:}

It is unclear if the greater radiological impact of tritium releases is as a normal effluent from an operating reactor or as a single, larger source term released during a transient. Tritium can be modeled in existing standalone codes which only consider tritium-specific transport phenomena and steady-state release as an effluent of a normal operating reactor. These codes often do not account for other reactor multiphysics effects but only require slight modification to account for design and material differences. Oppositely, if it is deemed necessary, tritium can be modeled within severe accident analysis or transient analysis codes if these transport models are integrated within them. Depending on the level of coupling, the formation, speciation, transport, material diffusion, and release from the sorbed state can be modeled in conjunction with other phenomena considered in these integral analysis codes. The impact of transient scenarios on tritium releases can be assessed.

Vendor discussions have indicated this to be a high priority task as there is a high degree of regulatory risk associated with tritium releases, and the true impact of tritium releases during 
various transient scenarios is currently unknown. It is recommended that a sensitivity study be completed for each reactor type to develop an understanding of the circulating inventory of tritium, the fraction removed by clean-up systems, the fraction trapped by specific materials, and the fraction released from those materials during various temperature transients and breaches in SSCs. Such a study would consist of a low amount of effort and would provide insights on the impact of tritium releases during potential accident scenarios. If the results of such a study show tritium releases during such scenarios are not of considerable concern, then it may not be needed to integrate tritium transport models into severe accident analysis codes such as MELCOR. This also has a near-term timeline in order to inform the need for the aforementioned code development items.

This resolution task is similar to those mentioned in the NRC's vision and strategy, which mentions the need to develop the SCALE code suite for streamlined tritium inventory estimations for FHR and MSR [2]. Additionally, there are development tasks recommended for tritium models within the MACCS code [2]. This includes atmospheric transport modeling and dosimetry updates. Dose consequence modeling for tritium releases is discussed more in Section D.4.

\begin{tabular}{|l|c|c|c|c|}
\hline Gap Resolution Action & Priority & Effort & Timeline & $\begin{array}{c}\text { NRC Vol3 } \\
\text { Crosscut }\end{array}$ \\
\hline $\begin{array}{l}\text { Evaluate the impact of tritium releases during } \\
\text { transient scenarios with sensitivity studies and } \\
\text { inventory calculations }\end{array}$ & High & Low & $\begin{array}{c}\text { Near- } \\
\text { term }\end{array}$ & $\begin{array}{c}\text { FHR-A2/D2, } \\
\text { MSR-CA4 }\end{array}$ \\
\hline
\end{tabular}

\section{B.8.2 Tool coupling}

Integrate tritium formation, transport, and release models into systems analysis and/or thermochemistry modeling tools:

There is considerable existing information regarding tritium adsorption, diffusion, transport, and release in graphite and other materials relevant to MSRs. Most of these phenomena have been incorporated into a number of models developed for nuclear systems, including fusionspecific modeling tools. Incorporating these physical models into MSR-specific tools such as systems analysis (e.g., SAM) or severe accident analysis (e.g., MELCOR) codes, and, more importantly, coupling the models to other phenomena such as thermochemistry will be important. Tritium has an impact on the redox potential of the salt which has a strong correlation not just to corrosion, but also radionuclide evaporation and transport. Therefore, it is recommended to couple tritium-specific models with electrochemical and thermochemical modeling tools to better understand the impact of tritium on fission product speciation, corrosion, deposition, and other aspects of radionuclide transport.

Vendor discussions have indicated this to be a medium priority as some designs are not expecting to have large tritium source terms due to fuel salt design choices. There is also question as to the radiological impact of tritium relative to other radionuclides, and therefore this is recommended as a long-term development objective rather than a near-term priority task. Depending on the results of the study proposed in Section B.8.1, there may not be a need to integrate these tritium models within severe accident analysis codes such as MELCOR, although the framework to do so exists. It should be noted that there is considerable motivation 
to develop these modeling tools for other phenomenological areas such as corrosion and salt chemistry, but the urgency in this development task for tritium source term modeling specifically is only rated at a medium level.

The effort involved with this task is rated as medium as work is already underway with incorporating tritium transport models into codes such as SAM, but refinement and validation of the models with existing data will be needed to maintain quality assurance. Additionally, thermochemistry coupling does not appear to be incorporated yet but will require further development of tasks outlined in Section B.4.1.

\begin{tabular}{|l|c|c|c|c|}
\hline Gap Resolution Action & Priority & Effort & Timeline & $\begin{array}{c}\text { NRC Vol3 } \\
\text { Crosscut }\end{array}$ \\
\hline $\begin{array}{l}\text { Integrate tritium formation, transport, and release } \\
\text { models into systems analysis and/or } \\
\text { thermochemistry modeling tools }\end{array}$ & Med & Med & $\begin{array}{c}\text { Long- } \\
\text { term }\end{array}$ & \\
\hline
\end{tabular}

\section{B.8.3 Release models}

\section{Complete experimental studies on tritium releases from graphite and integrate models into computational tools:}

It is recommended that experimental efforts be undertaken to better elucidate the release rate of tritium from core materials, especially graphite. The slow release of tritium from core graphite will need to be modeled to accurately account for the normal operation tritium effluent source term, but there is also concern as to the extent of release during a temperature transient such as that which may be seen in an accident scenario. A bounding estimate might include modeling a reactor's total tritium source term released from core graphite as a function of the temperature transient and into the environment. This would require accurate release coefficients. If there is a lack of such data in literature, measuring tritium release data with experiments is thus recommended. Ultimately, this would allow for accurate modeling of tritium releases not only during normal operation, but also during various types of accident scenarios where releases have a strong temperature dependence.

Vendor discussions have indicated this to be a medium priority due to the limited number of designs involving graphite and a considerable tritium source term. Additionally, the priority level for this resolution will be dependent on the results from the study proposed in Section B.8.1. Because there may be an experimental component to this development task, the effort required is rated as medium and this may require a long-term timeline for completion.

Assuming such data exists or can be found, it is imagined that minimal further development is needed as the release coefficients can be used by existing model frameworks within MELCOR for radionuclide releases from a material.

\begin{tabular}{|l|c|c|c|c|}
\hline Gap Resolution Action & Priority & Effort & Timeline & $\begin{array}{c}\text { NRC Vol3 } \\
\text { Crosscut }\end{array}$ \\
\hline $\begin{array}{l}\text { Complete experimental studies on tritium releases } \\
\text { from graphite and integrate models into } \\
\text { computational tools }\end{array}$ & Med & Med & $\begin{array}{c}\text { Long- } \\
\text { term }\end{array}$ & \\
\hline
\end{tabular}

\section{B.9Auxiliary System Modeling}


One of the biggest source term release concerns of vendors is the large amount of radioactivity which is stored outside the primary loop in various auxiliary systems such as the off-gas or cover gas clean-up system, or a fuel processing or polishing system, if used. It is difficult to discuss generalized designs of such systems for all proposed MSR designs as these systems can be as unique as the reactors themselves. Therefore, in this document, the off-gas system is meant to include any system where a cover gas or off-gas is removed from the primary loop (or fuel salt tubes/piping) and stored and transferred between various stages of chemical or physical processing. The fuel processing system is meant to include any system where the fuel salt itself is chemical or physically processed outside the primary loop and a portion or all of the processed fuel salt is returned to the primary loop.

From a modeling perspective, both of these types of auxiliary systems are similar and sometimes the same tool can be used for both to track materials throughout the specific design, but such a tool will need to account for several phenomena. This includes radioactive decay (parent decay and daughter formation) and chemical and physical processing that is both timeand location-dependent within the system. Relative to other source term modeling areas, this is not a demanding computational development task. The challenging aspect of this gap resolution has to do with the actual design of the auxiliary system, and specifically improving confidence in the removal constants which are crucial to accurately modeling the transport of radionuclides out of the primary loop and throughout the auxiliary system during normal operation. Understandably, some of the development tasks overlap with those also relevant to salt chemistry (fission product solubility, vaporization, deposition, etc.). Additionally, many source term concerns relate to accident scenarios involving the auxiliary systems, therefore effort should be allocated to understanding failure modes of proposed off-gas and fuel processing systems, as these can store large amounts of radioactivity in various chemical forms at any given moment.

\section{B.9.1 Off-gas and fuel processing system modeling tools}

\section{Develop modeling tools which track radionuclide flows throughout various stages of a custom auxiliary system:}

A complete mechanistic source term analysis of an MSR should track radionuclides beyond the primary loop such as the gaseous or aerosolized radionuclides which enter the off-gas system or the removal of certain undesirable elements in an on-line fuel processing system. These systems can represent large sources of radioactivity, and thus there is potential for a release to the environment. The individual processes themselves will need to be designed and analyzed with great detail (See Section B.9.2) but can be represented by a reduced-order model in a modeling framework designed to track all radionuclides throughout every stage of the system. Such a systems-level analysis tool can use removal constants to depict the transfer of radionuclides at certain stages, while also tracking decay to daughters, and other source or sink terms as needed.

Vendor discussion have indicated this to be a high priority modeling tool to be able to accurately track and account for radionuclides beyond the primary loop, throughout various custom-designed systems. The effort in developing such a modeling framework is rated as low due to the simplicity of the tool and similar efforts already published in literature. Some additional effort may be needed if it is desired to couple this framework with other phenomena 
such as core neutronics coupling (e.g., reactivity feedback effects). This development task should occur on a near-term timeline because there is a need for such a tool to begin source term tracking simulations. As will be discussed later, a refinement on the values of removal constants and the actual design of the auxiliary system does not need to be complete before such a tool is fully developed.

\begin{tabular}{|l|c|c|c|c|}
\hline Gap Resolution Action & Priority & Effort & Timeline & $\begin{array}{c}\text { NRC Vol3 } \\
\text { Crosscut }\end{array}$ \\
\hline $\begin{array}{l}\text { Develop modeling tools which track radionuclide } \\
\text { flows throughout various stages of a custom } \\
\text { auxiliary system }\end{array}$ & High & Low & $\begin{array}{c}\text { Near- } \\
\text { term }\end{array}$ & \\
\hline
\end{tabular}

\section{B.9.2 Off-gas system design and analysis}

\section{Integrate off-gas system research and development into modeling tools:}

Nearly every proposed MSR design is featuring some sort of system which carries off-gas from the primary fuel salt into some secondary loop or secondary area of the reactor system. As many fission products are volatile, and aerosol mists may also form and transport through the gas space, a proper understanding of the various process elements of the off-gas system is necessary to accurately model those treatment stages. Specifically for treatments involving separations, it is necessary to quantify these treatment stages by a removal constant which represents the separation of a certain element by a certain chemical or physical process. Even for treatment stages where the radionuclide is merely being held up, it is still necessary to understand the potential for unintentional release of the radionuclide from the stage. For example, charcoal beds may be used to hold-up noble gases, but the characteristics of potential release of the gas from the bed as a function of temperature should be investigated. Accident scenario modeling (See Section B.10) cannot be accomplished without an understanding of the failure modes of the off-gas or fuel processing system stages, which is a common concern amongst vendors.

Vendor discussions have indicated this to be a high priority gap resolution due to the level of gaseous radioactivity that the off-gas system will contain at any point during normal operation. This resolution task involves analyzing the ongoing and continued experimental efforts spanning multiple technical areas related to chemical separations and off-gas instrumentation design. Analyzing such experimental efforts and integrating that data into the modeling tool developed in Section B.9.1 will require a medium amount of effort given the wide variety of designs of such systems. Fortunately, recent work at ORNL has been underway for several years on off-gas system design and development with support from other DOE-NE programs, and this research program includes an emphasis on the source term. It is recommended that this work continues, with additional focus on improving confidence in the removal constants which represent the separations processes, and the potential failure modes of the SSCs involved. Because specific off-gas system design is still ongoing, the completion of this development task will require a long-term timeline dependent on the actual design of the overall reactor system as well as engineering challenges. But as the technology is developed, the integration into modeling tools can be accomplished in parallel. This resolution is in-line with that recommended for fission product transport chemistry modeling in the NRC's vision and strategy [2]. 


\begin{tabular}{|l|c|c|c|c|}
\hline Gap Resolution Action & Priority & Effort & Timeline & $\begin{array}{c}\text { NRC Vol3 } \\
\text { Crosscut }\end{array}$ \\
\hline $\begin{array}{l}\text { Integrate off-gas system research and } \\
\text { development into modeling tools }\end{array}$ & High & Med & $\begin{array}{c}\text { Long- } \\
\text { term }\end{array}$ & MSR-M3.2 \\
\hline
\end{tabular}

\section{B.9.3 Noble metal behavior in off-gas system}

Investigate noble metal behavior in molten salts, gas phase interactions:

There is much evidence from the operation of MSRE that some noble metals, including $\mathrm{Nb}$, $\mathrm{Mo}, \mathrm{Tc}, \mathrm{Ru}, \mathrm{Sb}$, and $\mathrm{Te}$, transported through and deposited on the off-gas lines. The term "noble metal" is used loosely by MSRE literature as some of these elements are not considered to noble metals, but rather transition metals or metalloids. Nonetheless, the designation refers to the inconsistent chemical behavior of this group in molten salt solutions, largely dependent on the redox potential of the system, as well as the physical behavior of the thermal fluid. The noble metals play a role in bubble transport (Sections B.5) as well as aerosol formation and transport (Section B.7), therefore it is conceivable how some of these radionuclides (and their daughters) were found deposited in off-gas lines. It is recommended that experimental efforts be undertaken to investigate the behavior of these transition metals and metalloids in molten salt solutions of varying redox potential. It may be advisable to include them in quantifiable concentrations in salt spill experiments and aerosol experiments to help elucidate these phenomena. Simple thermal analysis experiments can also be designed to investigate the behavior of these elements in specific salt compositions in various temperature regimes.

Vendor discussions have indicated this to be a medium priority resolution as there are still many unknowns regarding how to predict and model noble metal behavior, but the impact on source term is also still unknown. Multiple vendors have expressed concern about the inability to confidently predict the behavior of noble metals in their designs. The impact of redox potential, salt composition, and temperature on noble metal volatility, aerosol formation, and bubble transport needs to be investigated further. Because there are several unknowns regarding experimental design and the types of information desired, the effort required to complete this task is high. Studies on this topic are recommended to begin in the near-term but a full research program may take multiple years for complete resolution, therefore a long-term timeline is recommended. Ultimately, the results of which may have a considerable impact on design choices and source term analyses.

\begin{tabular}{|l|c|c|c|c|}
\hline Gap Resolution Action & Priority & Effort & Timeline & $\begin{array}{c}\text { NRC Vol3 } \\
\text { Crosscut }\end{array}$ \\
\hline $\begin{array}{l}\text { Complete experimental investigations into noble } \\
\text { metal chemistry in molten salts as well as gas } \\
\text { phase interactions }\end{array}$ & Med & High & $\begin{array}{c}\text { Long- } \\
\text { term }\end{array}$ & \\
\hline
\end{tabular}

\section{B.10 Accident Scenario Modeling}

Accident scenario modeling is a very important aspect of the licensing process for any nuclear reactor. While MSRs benefit from several inherent safety features which may limit the energy 
with which source term releases occur, the occurrence of a circulating inventory of radionuclides in both liquid and gas form does not preclude MSRs from having significant source term release scenarios during accidents. The use of defense-in-depth principles minimizes the probability and extent of radioactivity releases, but the use of methods in safety analysis and probabilistic risk analysis should be carried out to quantify the risks and consequences. Several broad examples of accident scenarios are covered here and recommendations are made as to how modeling and experimental efforts can be made to assess the associated source terms. These accidents include a generic salt spill from an SSC into a containment vessel outside the primary loop, a failure of an SSC separating two fluids leading to mixing, and a failure of an SSC in the off-gas system, potentially leading to a gaseous release to the next containment volume. Also briefly discussed is the failure of a solid fuel matrix containing TRISO fuel particles in an FHR, leading to solid fuel mixing in the molten coolant salt.

In addition to accidents during normal operation, there are scenarios where the fuel salt needs to be properly accounted for during shutdown or even decommissioning. This is in contrast to a solid fuel reactor where the solid fuel is not necessarily removed from the reactor core, and/or more easily tracked due to having a finite number of fuel rods. Some miscellaneous scenarios (not necessarily accidents) which are not covered here but need to be accounted for in a source term analysis include: incomplete draining of fuel salt from a reactor, leading to residual activity left in the primary loop; the shipping of portions of solidified spent fuel salt for various reasons; and the failure of an SSC during draining or shutdown, such as a freeze valve, which is instrumental to the draining of the salt from the primary loop.

\section{B.10.1 Salt spill}

The most obvious accident scenario involves the failure of an SSC and the resulting spilling, splashing, or spraying of the molten fuel salt into the next containment volume. It will be important to understand how this high temperature fluid will interact with the materials in that containment volume, as well as how it spreads and how quickly it cools. Additionally, the formation of aerosols is possible depending on the energy of the flow or spray. And finally, as the salt is cooling, some metal fluorides may volatilize as fluorine gas forms in the salt as a result of radiolysis (See Section B.4.4), which was evidenced by the investigation of the gas above the stored fuel salt 25 years after the shutdown of MSRE [22]. This experimental investigation should also include the interaction of the spilled salt with air or water during potential ingress into the containment volume, even though it may be proposed as being inert under normal operating conditions.

Vendor discussions have indicated this to be a high priority as many types of information will be gleaned from these experiments, including thermal fluids behavior, material interactions, aerosol formation, fission product evaporation, and metal fluoride volatilization. Integral effects tests are crucial to understanding molten salt reactor systems, and some phenomena may have heightened importance when tested in such experiments. Fortunately, this is very cross-cutting research and will support multiple research programs, and is already ongoing under the ART MSR Campaign [21]. The results of these experiments will support MELCOR development for modeling salt spills in generic geometries. 


\section{B.10.2 Salt mixing due to SSC breach or failure}

In the event of a failure of an SSC such as a heat exchanger, the fuel salt will mix with the primary coolant salt, potentially resulting in a chemical reaction. If the two fluids are different salts, or if they are two different types of fluids, then any chemical reactions and resulting speciation of the radionuclide will need to be assessed. If the two fluids are similar salts (e.g., both chloride salts), then speciation of the radionuclide is less of an unknown, but the resulting distribution of the radionuclide across the two streams will be important to estimate as the source term will be spread to a new loop and region of the reactor system. This gap resolution should also include the interaction of the salt with water as a result of an SSC. Specifically, this can include the ingress of water to the primary loop of the reactor, mixing with the molten fuel salt. This is in contrast to the addition of molten fuel salt to the containment volume which may contain some amount of water as a result of water ingress from a breach of containment. Both the addition of molten salt to water and water to molten salt should be investigated, accounting for different accident scenarios.

Vendor discussions have indicated this to be a medium priority, as many designs will utilize a similar coolant salt as the fuel salt, and thus a chemical reaction resulting from mixing is less of a concern. The interaction of the salt with water may be a higher priority, but may not be plausible for all designs, and so the priority is maintained at a medium level. Some vendors have noted the possibility of fuel salt mixing with molten hydroxide which may be used in different aspects of the reactor. Accounting for all possible unintended fluid mixing scenarios will be important in completing an analysis of this accident scenario.

\section{B.10.3 Off-gas system breach or failure}

It may be desirable to investigate specific accident scenarios involving a proposed or representative off-gas system for a generic MSR in order to better understand the risks and consequences involved. Such initiating events can include the failure of an SSC in the off-gas system where radionuclides may transport through or be stored in, or it may include the failure of an SSC external to the off-gas system, allowing air ingress. Some off-gas systems may be designed to operate in inert environments, therefore potential reactions of system components with air should be considered. Additionally, this air ingress may allow a simple transport pathway to the environment.

Vendor discussions have indicated this to be a high priority as the off-gas system may contain a relatively large amount of radioactivity in gaseous form. Several vendors have noted the concern of a potential release from the off-gas system, and the unknowns involved with an air or water ingress. Given a certain off-gas system design and preliminary details about the potential SSCs, a preliminary safety analysis of the system may be attempted to help guide the system design. An analysis of this accident scenario should occur in parallel to the work described in Section B.9.2 consisting of the design and analysis of the off-gas system.

\section{B.10.4 Damage to fuel matrix (FHR only)}

Understanding the likelihood of damage to the fuel matrix holding the TRISO particles is important, as this can change the geometry and configuration of the fuel. If a fuel pebble 
matrix or prismatic matrix were to spill TRISO fuel particles out somehow, a neutronics analysis of the resulting fuel geometry in the coolant salt or other environment will be necessary to maintain subcritical geometries as well as quantify releases. The interaction of TRISO fuel with molten fluoride salts have been investigated some, but there are still unknowns regarding the extent of radionuclide release from the particle layers. Damage to the fuel matrix can be caused due to mechanical stress or due to oxidation of the graphite under favorable conditions. The thermal response of the fuel matrix and fuel particles can also be modeled during temperature transients.

Vendor discussions have indicated this to be a low priority as the risk of failure and corresponding consequence are small relative to other accident scenarios. To investigate this accident scenario, it would be necessary to model the failure of a fuel matrix, as well as the resulting salt-TRISO interactions and the thermal response of the fuel particle and core materials during potential temperature transients. The thermal response modeling has also been outlined in the NRC's code development vision and strategy, Vol. 3 [2]. Such heat transfer modeling of the fuel components will be accomplished in MELCOR. 


\section{Sodium-Cooled Fast Reactors}

\section{C.1 Core Neutronics and Fuel Depletion}

SFR neutronics and fuel depletion calculations are well established, given the U.S.'s long history of sodium reactor development. As outlined in ref [5], multiple codes are available to perform the analyses necessary to develop the initial radionuclide inventory of the fuel, as an input for source term calculations. In discussions with industry, no additional gaps or immediate actions were identified.

\section{C.2 Radionuclide In-Pin Migration and Release}

For SFR metallic fuel, radionuclide migration within the fuel pin during irradiation has a significant impact on the availability of radionuclides for release during cladding failure events. This burnup dependence, in addition to temperature conditions at the time of clad failure, are of foremost importance for the modeling of radionuclide release from the fuel during transient event sequences. Past studies have examined these phenomena in detail, including a review of available data, identification of gaps, and prioritization of resolution activities [23, 42]. The findings of these studies, in addition to recent conversations with industry, were used to outline the resolution actions outlined in this subsection.

\section{C.2.1 Metal fuel post-irradiation examination (PIE)}

\section{Addressing data gaps regarding radionuclide migration through additional metal fuel PIE:}

As part of the data collection efforts and analysis in refs [23, 42], several gaps were noted on the available data regarding the in-pin migration behavior of certain radionuclides (iodine, strontium, barium, europium, and tellurium). The uncertainty associated with the migration of these elements can result in the use of conservative assumptions regarding radionuclide release fractions from the fuel. As a result, greater emphasis is placed on the performance of subsequent radionuclide barriers (the sodium pool, reactor vessel head, and containment/confinement). Improved data in this area and the reduction of uncertainties could help relax requirements for these barriers.

Irradiated metal fuel post-irradiation examination (PIE) is necessary to obtain additional data on radionuclide migration during irradiation. The most likely sources would be irradiated EBRII or FFTF (IFR-1 and MFF series) fuel and recent AFC experiments, although international sources (such as METAPHIX) are also possible. Similar work has been performed recently at the Materials and Fuels Complex at INL, which greatly contributed to the assessment of metal fuel radionuclide migration provided in ref [42].

This task is considered medium priority and a long-term need, as the existing gap can be addressed through the use of conservative assumptions to allow the completion of MST analyses. However, the assumptions do impact subsequent phases of the analysis. The effort level is designated as high, as PIE of irradiated fuel requires significant planning and resources. Improving the existing database regarding radionuclide migration would also support the development and validation of the SFR fuel models within MELCOR (SFR - M1.2). 


\begin{tabular}{|l|c|c|c|c|}
\hline Gap Resolution Action & Priority & Effort & Timeline & $\begin{array}{c}\text { NRC Vol3 } \\
\text { Crosscut }\end{array}$ \\
\hline $\begin{array}{l}\text { Additional metal fuel PIE to address data gaps } \\
\text { regarding metal fuel radionuclide migration }\end{array}$ & Med & High & $\begin{array}{c}\text { Long- } \\
\text { term }\end{array}$ & SFR-M1.2 \\
\hline
\end{tabular}

\section{C.2.2 Radionuclide migration modeling during pre-transient irradiation}

Assess the capabilities of high-fidelity fuel performance modeling tools for the analysis of in-pin radionuclide migration during pre-transient irradiation:

While the migration of radionuclides within the fuel pin during irradiation is one of the factors that dictates radionuclide release with cladding failure, the U.S. currently does not have a computer code that mechanistically models the movement of the radionuclides that are important for source term analyses. The Argonne SRT code uses data-driven models utilizing user input to approximate this behavior, based on the findings of past studies [43]. However, higher-fidelity fuel performance codes and models exist that may offer a platform for a detailed assessment of radionuclide migration.

Within the SAS4A/SASSYS-1 code, the SSCOMP-A model assesses changes in the metal fuel that occur during irradiation. This includes the migration and redistribution of fuel constituents, such as uranium, plutonium, and fission gases. The information calculated by SSCOMP-A is utilized to characterize the local thermo-physical properties of the fuel (conductivity, melting point temperatures, etc.), which are important fuel for the determination of the fuel temperatures, fission gas pressure, and fuel pin failure [44]. However, not all of the radionuclides important for source term analysis are currently within the code, since the focus of the assessment is fuel performance. Similarly, the NEAMS fuel performance tool BISON is capable of modeling metal fuel behavior during irradiation, including constituent redistribution and fission gas release [45]. As of yet, BISON has not been utilized for the tracking of radionuclides important to source term analysis. As part of this resolution pathway, the capabilities of both SSCOMP-A and BISON would be assessed for a potential extension to mechanistically track the migration of radionuclide important for source term analysis, including the possibility to develop reduced-order models based on their results.

This resolution action is considered medium priority. Although data-driven models can be utilized currently to complete MST assessments, they are limited by the available supporting data and could result in conservative assumptions (see C.2.1). The action is considered a nearterm need as it is a predecessor activity that focuses on an assessment of the models to be adapted for this problem, with a potential for subsequent model development. The required effort is considered low for similar reasons. Lastly, this action potentially crosscuts with SFRM1.2 from NRC Vol. 3, as the insights from the high-fidelity codes may be used in the development of MELCOR fuel models.

\begin{tabular}{|l|c|c|c|c|}
\hline Gap Resolution Action & Priority & Effort & Timeline & $\begin{array}{c}\text { NRC Vol3 } \\
\text { Crosscut }\end{array}$ \\
\hline $\begin{array}{l}\text { Assessment of high-fidelity fuel performance } \\
\text { codes for the analysis of in-pin radionuclide } \\
\text { migration during pre-transient irradiation }\end{array}$ & Med & Low & $\begin{array}{c}\text { Near- } \\
\text { term }\end{array}$ & SFR-M1.2 \\
\hline
\end{tabular}




\section{C.2.3 Fuel pin failure and fuel melting experiments}

Addressing data gaps through additional fuel pin failure and fuel melting experiments: As part of the data collection efforts and analysis in refs [23, 42], several gaps were noted on the available data regarding radionuclide release from high burnup, high temperature metallic fuel. This includes radionuclide release from molten metallic fuel pins within a sodium environment. While some data on radionuclide release from high temperature metal fuel is available, the conditions for many of the tests were not prototypic of SFR transient event sequences. In addition, although several past sodium cooled reactor accidents have resulted in the melting of metal fuel and relocation of fuel into the sodium coolant, the low burnup of the fuel at the time limits the insights that can be gained.

The closure of this gap would likely require experiments involving the melting of irradiated metallic fuel within a sodium environment. Such conditions have previously been achieved through testing in TREAT, however the focus of the tests was on fuel performance rather than the tracking of released radionuclides [46]. Given the complexities involved with such tests, the effort for this task is considered high and a long-term activity. A medium priority designation is assigned for several reasons. First, conservative assumptions can be utilized to allow the completion of MST analyses (with the associated repercussions outlined in C.2.1). In addition, the frequency of large-scale fuel melting events in metal fuel pool-type SFR designs is likely to be below the threshold that would require detailed MST assessments. However, the priority is raised to the medium-level as reducing the uncertainties related to fuel release fractions could impact design decisions and licensing analyses. Concerning MELCOR development, this task would support SFR-M1.2, which focuses on the development of fuel release models.

\begin{tabular}{|l|c|c|c|c|}
\hline Gap Resolution Action & Priority & Effort & Timeline & $\begin{array}{c}\text { NRC Vol3 } \\
\text { Crosscut }\end{array}$ \\
\hline $\begin{array}{l}\text { Metal fuel pin failure and melting experiments to } \\
\text { address data gaps regarding radionuclide release. }\end{array}$ & Med & High & $\begin{array}{c}\text { Long- } \\
\text { term }\end{array}$ & SFR-M1.2 \\
\hline
\end{tabular}

\section{C.2.4 Fuel Behavior and Radionuclide Relocation Modeling during Transient Scenarios}

\section{Assess the capabilities of high-fidelity fuel performance models for the analysis of radionuclide relocation during postulated severe accidents:}

As highlighted above, the release of radionuclides from failed metal fuel pins depends on both the pre-transient radionuclide migration and the behavior of the fuel during the transient scenario. Currently, the U.S. does not have a computer code that mechanistically models the relocation of those radionuclides important for source term analyses during the transient. Within the SRT code, this analysis is currently handled through data-tables based on available information. However, within the SAS4A/SASSYS-1 code, the fuel behavior and relocation phenomena during severe accidents are described in significant detail.

Within SAS4A/SASSYS-1, the fuel relocation phenomena during the accident are divided broadly into two categories: a) fuel behavior and in-pin relocation prior to cladding failure, and b) fuel ejection and relocation in the core coolant channels following the cladding failure. The metal fuel behavior and in-pin fuel relocation prior to cladding failure are described by the 
DEFORM5-A and PINACLE-M modules [44]. These models describe the migration and redistribution of fuel constituents, such as uranium, plutonium, and fission gases, fuel melting, and axial in-pin fuel relocation. The local fuel composition calculated by DEFORM5-A and PINACLE-M is utilized to characterize the local thermo-physical properties of the fuel (conductivity, melting point temperatures, etc.), which are important for the determination of the fuel temperature, fission gas pressure, and fuel pin failure.

The metal fuel and fission gas ejection and relocation in the coolant channels following the cladding failure are described by the LEVITATE-M module. LEVITATE-M describes the axial relocation of the molten fuel, fuel freezing on cladding and assembly walls, frozen fuel crust breakup and subsequent relocation of the frozen fuel particles. Multiple fuel components and fission gas, consistent with those tracked in SSCOMP-A, DEFORM5-A, PINACLE-M are tracked as they relocate in the coolant channel between the failure location and the sodium pool.

All the SAS4A metal fuel modules used for accident analysis track the behavior and relocation of multiple fuel components and fission gas consistent with those tracked in the pre-transient module SSCOMP-A. However, not all of the radionuclides important for source term analysis are currently tracked in the SAS4A metal fuel modules since the focus of the assessment is the reactor response during the transient for safety analyses.

As part of this resolution pathway, the capabilities of DEFORM5-A, PINACLE-M, and LEVITATE-M would be assessed for a potential extension to mechanistically track the relocation of radionuclides important for source term analysis, including the possibility to develop models that describe the radionuclide ejection into the coolant channel after cladding failure and the radionuclide deposition on various reactor core surfaces as they travel between the cladding failure location and the sodium pool.

This resolution action is considered medium priority. Although data-driven models can be utilized currently to complete MST assessments, they are limited by the available supporting data and could result in conservative assumptions (see C.2.1). The addition of mechanistic models that quantify the actual quantity of radionuclides ejected from the fuel pin into the coolant channel and the radionuclide deposition on various core surfaces as they travel between the failure location and the sodium pool could reduce this conservatism by providing a more accurate estimate of the amounts of radionuclides that reach the sodium pool. This action is considered a near-term need, as this focuses on an assessment of the models to be adapted for this problem, with a potential for subsequent model development. The required effort is considered low for similar reasons. Lastly, this action potentially crosscuts with SFR-M1.2 from NRC vol 3, as the insights from the high-fidelity codes may be used in the development of MELCOR metal fuel release models.

\begin{tabular}{|l|c|c|c|c|}
\hline Gap Resolution Action & Priority & Effort & Timeline & $\begin{array}{c}\text { NRC Vol3 } \\
\text { Crosscut }\end{array}$ \\
\hline $\begin{array}{l}\text { Assessment of high-fidelity fuel performance } \\
\text { codes for the analysis of in-pin and ex-pin } \\
\text { radionuclide relocation during transient scenarios. }\end{array}$ & Med & Low & $\begin{array}{c}\text { Near- } \\
\text { term }\end{array}$ & SFR-M1.2 \\
\hline
\end{tabular}




\section{C.3 Sodium Pool Chemistry}

\section{C.3.1 Thermochemical modeling}

\section{Assess the readiness of the current SFR thermodynamic database for direct integration into source term modeling tools:}

As part of recent DOE-funded SFR source term analysis efforts [23], a database was developed of available chemical thermodynamic data for radionuclide behavior in sodium. This database and the thermodynamic equilibrium solver HSC Chemistry [47] was used to develop vapor fraction functions that have been instituted into the SRT code. However, tools such as MELCOR are now looking to directly integrate the thermodynamic equilibrium solver into the code itself. This task focuses on the assessment of the current database for its readiness to be directly incorporated into a source term analysis tool. The assessment would identify any data gaps, potentially influential phenomena not currently modeled (see C.3.2), and the formatting of the information.

This task is considered medium priority, as radionuclide vaporization from the sodium pool was found to be of relatively minor impact in recent studies [23], however, this is an important step in the continued development of MELCOR for SFRs. The effort level is considered low, as this is an assessment of an existing database at a national laboratory, and a near-term need. This task would be in support of MELCOR task M1.3.

\begin{tabular}{|l|c|c|c|c|}
\hline Gap Resolution Action & Priority & Effort & Timeline & $\begin{array}{c}\text { NRC Vol3 } \\
\text { Crosscut }\end{array}$ \\
\hline $\begin{array}{l}\text { Assess the readiness of the current SFR } \\
\text { thermodynamic database for direct integration } \\
\text { into source term modeling tools. }\end{array}$ & Med & Low & $\begin{array}{c}\text { Near- } \\
\text { term }\end{array}$ & SFR-M1.3 \\
\hline
\end{tabular}

\section{C.3.2 Kinetic and non-homogeneous effects.}

\section{Addressing data gaps on kinetic and non-homogeneous mixing effects through experimentation:}

As highlighted in the preceding resolution, several past efforts on SFR source term analyses have utilized thermodynamic equilibrium calculations to determine the speciation of radionuclides within the sodium pool and their potential to vaporize from the sodium [23, 48]. Models based on this analysis approach are also utilized within the SRT source term analysis code and a thermodynamic equilibrium solver is planned for MELCOR. However, some experimental evidence exists indicating the non-homogeneous mixing may occur for certain radionuclides in sodium $[49,50]$. In addition, thermodynamic equilibrium does not account for possible kinetic effects during the formation of compounds.

This resolution would seek to conduct a series of small experiments utilizing non-radioactive isotopes of critical elements to identify influential kinetic and non-homogeneous impacts on radionuclide behavior within liquid sodium. The results of such experiments would be utilized to improve the existing pool transport and vaporization models within MELCOR and SRT. This action is considered low priority based on the findings or ref [23], as sodium pool vaporization was not identified as a major influence on offsite dose results. However, if new 
data suggests that kinetic or non-homogeneous effects could significantly impact vaporization behavior, that ranking would need to be revised. The associated effort is considered medium, as experimentation with sodium is necessary, but could likely be achieved at bench-scale. Finally, this is considered a long-term activity, as the extent of future testing needed is not clear at this time.

\begin{tabular}{|l|c|c|c|c|}
\hline Gap Resolution Action & Priority & Effort & Timeline & $\begin{array}{c}\text { NRC Vol3 } \\
\text { Crosscut }\end{array}$ \\
\hline $\begin{array}{l}\text { Addressing data gaps on kinetic and non- } \\
\text { homogeneous mixing effects of radionuclides in } \\
\text { sodium through experimentation. }\end{array}$ & Low & Med & $\begin{array}{c}\text { Long- } \\
\text { term }\end{array}$ & SFR-M1.3 \\
\hline
\end{tabular}

\section{C.4 Bubble Transport}

A potential avenue for radionuclides released from the fuel to bypass retention within the sodium pool is through transport within fission gas bubbles. Past studies have identified this transport pathway as a high priority development area, given its bypass characteristics and a general lack of validation data [23]. Although mechanistic bubble transport models exist within advanced reactor source term tools, such as SRT and MELCOR, experimental data is needed to provide confidence in the model results.

Based on the recommendations of ref [23], DOE initiated experimental efforts to examine bubble transport within liquid sodium and lead as part of the NEUP program [51, 52]. These efforts, taking place at the University of Wisconsin and University of New Mexico, are scheduled to conclude within the next year. Validation efforts utilizing the experimental results have already begun [53] and will continue as the experiments complete. In addition, the validation efforts also form the basis of an international benchmark exercise with Japan as part of the Civil Nuclear Energy Research and Development Working Group (CNWG).

Given the high priority of this development area and the resources invested by the DOE, completion of the experiments and the further development, refinement, and validation of bubble transport models is a critical near-term task. It is also an important factor in the completion of MELCOR development pathway SFR-M1.3, which focuses on radionuclide transport through the sodium pool.

\begin{tabular}{|l|c|c|c|c|}
\hline Gap Resolution Action & Priority & Effort & Timeline & $\begin{array}{c}\text { NRC Vol3 } \\
\text { Crosscut }\end{array}$ \\
\hline $\begin{array}{l}\text { Completion of NEUP bubble transport in sodium } \\
\text { and lead experimental efforts and validation of } \\
\text { bubble transport models }\end{array}$ & High & Low & $\begin{array}{c}\text { Near- } \\
\text { term }\end{array}$ & SFR-M1.3 \\
\hline
\end{tabular}

\section{C.5 Aerosol Behavior}

\section{C.5.1 Sodium aerosols}

Assessment of available sodium aerosol data and model validation efforts:

Radionuclide aerosol modeling for SFRs is largely dependent on the behavior of sodium aerosols, which are the dominant species within the reactor vessel cover gas region. Any 
subsequent leakage from this volume into containment/confinement and further transport will likely be dictated by the evolution of sodium aerosols and their interaction with oxygen and water vapor.

Aerosol deposition modeling is a field of extensive study and existing source term analysis tools, such as MELCOR and SRT, contain models to capture this behavior. The focus of this task is the assessment and collection of available sodium aerosol data for the validation of these models. This task is already underway as part of MELCOR development for the NRC (SFR-M1.5) and SRT development for use as part of the safety basis for the Versatile Test Reactor (VTR). The goal of this recommendation is the coordination of efforts and publication of results to support the further use of these codes for reactor licensing and authorization activities. In addition, any gaps in existing data will be identified, such as the potential impact of chemical reactions that are not currently included within the models, with the development of plans for resolution. The task is given a medium priority, based on the importance assessment and sensitivity analyses conducted in ref [23].

\begin{tabular}{|l|c|c|c|c|}
\hline Gap Resolution Action & Priority & Effort & Timeline & $\begin{array}{c}\text { NRC Vol3 } \\
\text { Crosscut }\end{array}$ \\
\hline $\begin{array}{l}\text { Assessment of available data on sodium aerosol } \\
\text { behavior and publication of model validation } \\
\text { efforts. }\end{array}$ & Med & Low & $\begin{array}{c}\text { Near- } \\
\text { term }\end{array}$ & SFR-M1.5 \\
\hline
\end{tabular}

\section{C.5.2 Leakage and pathway plugging behavior}

\section{Assessment of available data and development of models for the analysis of pathway plugging due to sodium aerosols and vapors:}

When sodium aerosols and vapors transport from an inert gas environment to a volume that contains oxygen and water vapor, there is a potential for pathway plugging due to the formation of sodium oxides. This phenomenon is particularly important for the transport of radionuclides from the cover gas region of the reactor vessel through leakage pathways in the reactor head to the containment/confinement volume. The ability to credit the reduction in leakage pathway area due to plugging can assist in a reduction in the magnitude of radionuclides entering the next volume. Although models to capture plugging behavior have been proposed [54], this phenomenon is not currently included within SRT or the MELCOR SFR models. Past U.S. experimentation on leak plugging is available (see ref [54] for a summary), along with more recent international efforts [55].

This resolution is divided into two tasks. The goal of the first task, which is considered a low effort near-term task, is to assess the available data and determine its adequacy for model development. Based on these findings, a follow-on task focuses on the development and incorporation of a model for leak plugging due to sodium aerosols and vapors. If the available is found insufficient, plans for the closure of data gaps will be developed. Overall, these are considered medium priority tasks, as the phenomenon can be treated through assumptions, but further refinement could alleviate the requirements associated with containment/confinement design. MELCOR development item SFR-M1.5, which centers on containment modeling, would be supported by this task. 


\begin{tabular}{|l|c|c|c|c|}
\hline Gap Resolution Action & Priority & Effort & Timeline & $\begin{array}{c}\text { NRC Vol3 } \\
\text { Crosscut }\end{array}$ \\
\hline $\begin{array}{l}\text { Assessment of available models for the analysis } \\
\text { of pathway plugging due to sodium aerosols and } \\
\text { vapors. }\end{array}$ & Med & Low & $\begin{array}{c}\text { Near- } \\
\text { term }\end{array}$ & SFR-M1.5 \\
\hline $\begin{array}{l}\text { Development and incorporation of pathway } \\
\text { plugging models }\end{array}$ & Med & Med & $\begin{array}{c}\text { Long- } \\
\text { term }\end{array}$ & SFR-M1.5 \\
\hline
\end{tabular}

\section{C.6 Sodium Fires}

\section{C.6.1 Radionuclide release}

\section{Assessment of available data and development of models regarding radionuclide release during sodium fires:}

In certain sodium fire scenarios, such as those involving leaks from sodium cleanup systems, there is a potential to release radionuclides that are contained within the liquid sodium. Recent work on MELCOR development has centered on the addition of sodium fire models into the code, however, the focus has been on the thermal effects of the fire rather than radionuclide release. This gap resolution seeks to support the expansion of MELCOR capabilities to capture radionuclide releases during sodium fires.

As with the preceding gap resolution, this resolution is divided into two tasks. A near-term, low-effort task focuses on the collection and assessment of available data regarding radionuclide release during sodium fires. Based on these findings, models will be developed and incorporated into MELCOR as part of a long-term activity. If the available is found insufficient, plans for the closure of data gaps will be developed. These are considered medium priority items, as potential radionuclide releases from sodium clean-up systems during leaks and fires may be relatively high frequency SFR radionuclide release event sequences (in comparison to core fuel damage events). However, it is straightforward to apply conservative assumptions if detailed models are not available. These activities directly support MELCOR activities SFR-M1.5 and M1.6 on containment and sodium fire modeling.

\begin{tabular}{|l|c|c|c|c|}
\hline Gap Resolution Action & Priority & Effort & Timeline & $\begin{array}{c}\text { NRC Vol3 } \\
\text { Crosscut }\end{array}$ \\
\hline $\begin{array}{l}\text { Assessment of available data on radionuclide } \\
\text { release from sodium fires }\end{array}$ & Med & Low & $\begin{array}{c}\text { Near- } \\
\text { term }\end{array}$ & $\begin{array}{c}\text { SFR-M1.5 } \\
\text { and M1.6 }\end{array}$ \\
\hline $\begin{array}{l}\text { Development and incorporation of models for } \\
\text { radionuclide release during sodium fires }\end{array}$ & Med & Med & $\begin{array}{c}\text { Long- } \\
\text { term }\end{array}$ & $\begin{array}{c}\text { SFR-M1.5 } \\
\text { and M1.6 }\end{array}$ \\
\hline
\end{tabular}

\section{C.7 Accident Scenario Modeling}

In general, SFR transient scenario modeling is well established due to extensive past efforts in support of SFR design and licensing. However, there are several key areas that potentially impact MST analyses where further refinement would be beneficial. First, improved modeling of fuel behavior during fuel pin failure and melting events would improve MST insights (as outlined in Section C.2.4). In addition, insights into possible ex-core accident scenarios, such as fuel handling errors or potential leaks and fires associated with sodium clean-up systems, would provide useful for outlining the flexibility needed in MST modeling tools. 


\section{C.8 Lead-Cooled Fast Reactor Items}

As many of the tasks necessary for the development of LFR source term models mirrors those for SFRs, separate gaps resolution activities are not provided. However, there are several areas worthy of highlight. First, although some LFR designers are proposing to use oxide fuel initially, which has well developed radionuclide release models, there is a hope to transition to nitride or bondless metal fuel in the future. As these designs mature, there may be a need for additional radionuclide migration and release experiments for the novel fuel types.

The tasks outlined above for sodium pool bubble transport and pool vaporization are similar for LFRs. As mentioned in Section C.4, there is ongoing experimentation under the NEUP program on radionuclide behavior in lead, which will develop vital validation data for codes such as MELCOR, SRT, and the FATE code developed by FAI [56]. Lastly, when possible, coordination with international LFR projects (such as those being pursued by European Union in support of ALFRED [57]) can assist in expediting development efforts and avoiding duplicative work. 


\section{Consequence Modeling}

Several previous studies have been performed in order to assess the capabilities to model the consequences of an advanced reactor accident. In general, these previous studies examined the capabilities of the MELCOR Accident Consequence Code System (MACCS) and concluded the flexibility in the code is well suited for advanced reactor applications. However, several knowledge gaps and improvement areas do exist in order to best tailor consequence analysis codes for advanced reactor applications. The purpose of this section is to discuss these topics. The following sections are extracted from ref [7] and are consistent with the findings of these previous studies, with much of the information gathered directly from ref [39].

\section{D.1Near-Field Dispersion}

\section{D.1.1 Benchmarking}

\section{Benchmark against higher fidelity codes (or current regulator approved codes) for atmospheric dispersion:}

Near-field modeling refers to atmospheric transport and dispersion modeling within 500 meters of the source. Within 500 meters of the source, dispersion phenomena including building wake effects and potential for recirculation cavity zones can cause higher concentrations of released contaminants [2]. Currently in MACCS, a simple model is used for building wake effects which scales the dispersion parameters based on the building dimensions, which can lead to conservative estimates for concentrations in the near-field. With the expectation that advanced reactors will have smaller source terms compared to their LWR counterparts, higher fidelity atmospheric transport and dispersion models focused closer to the source is of particular importance. Such analysis can be influential in determining appropriate Emergency Planning Zones (EPZs) for advanced reactors, which may be more appropriately set at a distance below the 10 mile EPZ used for LWRs.

To assess the degree of conservatism in the near-field, SNL conducted a study in 2019 which compared MACCS v3.11.6 to several near-field atmospheric transport and dispersion codes including QUIC, ARCON96, and AERMOD2. This study concluded that MACCS performed comparably for near-field atmospheric transport and dispersion modeling, and provided a conservatively bounding assessment compared to QUIC, ARCON96 and AERMOD [58]. This study motivated near-field model improvements for MACCS described in Section D.1.2 below.

Further benchmarking could additionally be performed in order to compare currently approved codes for regulatory purposes to additional codes. For example, it could be demonstrated that ARCON96 and MACCS meet the requirements of Regulatory Guide 1.145 via comparison with PAVAN. MACCS 4.1 is scheduled for release in July 2021 and will contain added capabilities incorporating the models used for both ARCON96 and PAVAN, which could prove useful in performing additional model comparison studies. See D.1.2 for additional details. This item was rated as high priority and near-term due to feedback from industry and relevance to licensing submission. The effort level was rated as medium due to the extensive progress already made in refs [58] and [59] towards addressing this. 


\begin{tabular}{|l|c|c|c|c|}
\hline Gap Resolution Action & Priority & Effort & Timeline & $\begin{array}{c}\text { NRC Vol3 } \\
\text { Crosscut }\end{array}$ \\
\hline $\begin{array}{l}\text { Benchmark against higher fidelity codes (or } \\
\text { current regulator approved codes) for atmospheric } \\
\text { dispersion }\end{array}$ & High & Med & $\begin{array}{c}\text { Near- } \\
\text { term }\end{array}$ & TN-CA1 \\
\hline
\end{tabular}

\section{D.1.2 Near-field model improvements}

Improving atmospheric transport and dispersion modeling to be less conservative and higher fidelity for near-field applications:

Informed by the 2019 near-field comparison study, the MACCS team is releasing MACCS v4.1 in July 2021 where the primary model enhancements are added options to better model near-field atmospheric dispersion. Added options include the ability to:

- $\quad$ Model plume meander using the Ramsdell and Fosmire model

- $\quad$ Model trapping and downwash using the Briggs model, with options for specifying building parameters or buoyancy flux

Implementing these options reduces the conservatism in concentration calculations in the nearfield, while also allowing MACCS users to match near field modeling results for both ARCON96 and PAVAN, given the proper parametrization [59]. However, these near-field modeling improvements currently only apply to the Gaussian Plume Segment model within MACCS. MACCS also integrates with HYbrid Single-Particle Lagrangian Integrated Trajectory (HYSPLIT) for a higher-fidelity Lagrangian particle tracking model, which is more accurate as it tracks individual particles rather than assuming a Gaussian distribution for the dispersion. The HYSPLIT model requires further refinement of the time stepping scheme and dispersion parameterization in order to be utilized for higher fidelity near-field modeling [39]. A recommended path forward to increase the fidelity of consequence modeling in the nearfield would include refining the time stepping scheme and dispersion parameterization in HYSPLIT and integrating this new capability into consequence analysis tools including MACCS, STORM, and Specialized Hazard Assessment Response Capability (SHARC) to increase the fidelity of near-field modeling [60].

Improving near-field modeling capabilities to be less conservative was rated as high priority and near-term due to feedback from industry and relevance to licensing submission, and was rated as medium due to the extensive progress already made in [58] and [59] towards addressing this. The improvements to HYSPLIT in order to increase the modeling fidelity in the near-field was rated as medium priority and medium effort due to direct tie to [2] and estimates provided in ref [60].

\begin{tabular}{|l|c|c|c|c|}
\hline Gap Resolution Action & Priority & Effort & Timeline & $\begin{array}{c}\text { NRC Vol3 } \\
\text { Crosscut }\end{array}$ \\
\hline $\begin{array}{l}\text { Improving atmospheric transport and dispersion } \\
\text { modeling to be less conservative for near-field } \\
\text { applications. }\end{array}$ & High & Med & $\begin{array}{c}\text { Near- } \\
\text { term }\end{array}$ & TN-CA1 \\
\hline $\begin{array}{l}\text { Increase near-field modeling fidelity by making } \\
\text { improvements to HYSPLIT }\end{array}$ & Med & Med & $\begin{array}{c}\text { Long- } \\
\text { term }\end{array}$ & TN-CA1 \\
\hline
\end{tabular}




\section{D.1.3 Near-field model improvements for dispersed source terms}

\section{Enhanced capability to model multiple source terms arising from spatially offset locations:}

Several advanced reactor designs propose two or more reactors per site, therefore the capability to model multiple source terms from distinct locations serves as a current gap. Current practice for LWRs is to model multiple source terms offset only by time, if necessary, but the location of the source term is set to a single location. There is the potential, especially for near-field calculations, for differences to arise in concentrations when multiple source terms are modeled arising from different spatial locations.

To accommodate the capability to model multiple source terms from different spatial locations, a high-level of effort would be required to modify the underlying spatial setup for current codes, from polar coordinated to cartesian coordinates. This item was rated as low priority and long-term because conservative bounding assessments may be used in the absence of this capability.

\begin{tabular}{|l|c|c|c|c|}
\hline Gap Resolution Action & Priority & Effort & Timeline & $\begin{array}{c}\text { NRC Vol3 } \\
\text { Crosscut }\end{array}$ \\
\hline $\begin{array}{l}\text { Enhanced capability to model multiple source } \\
\text { terms arising from spatially offset locations. }\end{array}$ & Low & High & $\begin{array}{c}\text { Long- } \\
\text { term }\end{array}$ & \\
\hline
\end{tabular}

\section{D.2Isotopic Inventory}

\section{D.2.1 Reactor-specific inventories}

Provide pre-loaded isotopic inventory files based on depletion analysis for the specific reactor type and core geometry of interest:

The source term, which characterizes the magnitude and timing of released material, represents the starting point for consequence analysis modeling. In order to characterize the magnitude and timing of the release and facilitate the translation of MELCOR data into use by MACCS, an accurate description of the isotopic inventory within the reactor is necessary. This isotopic inventory takes into account the unique chemical makeup of that specific reactor design, so that when mechanistic source term codes are applied in MelMACCS to generate the source terms for use in MACCS, the release fractions are applied to an accurate assessment of the radionuclides contained within that system.

This item was rated as high priority and near-term because characterizing the isotopic inventory is a prerequisite for follow-on activities, to include assessment of activation, released radionuclides, and released chemical forms. Resolving the gap would include SCALE modeling which is already in progress per [2] and is therefore rated as medium effort. 


\begin{tabular}{|l|c|c|c|c|}
\hline Gap Resolution Action & Priority & Effort & Timeline & $\begin{array}{c}\text { NRC Vol3 } \\
\text { Crosscut }\end{array}$ \\
\hline $\begin{array}{l}\text { Provide pre-loaded isotopic inventory files based } \\
\text { on depletion analysis for the specific reactor type } \\
\text { and core geometry of interest. }\end{array}$ & High & Med & $\begin{array}{c}\text { Near- } \\
\text { term }\end{array}$ & $\begin{array}{c}\text { SFR-CA2.1, } \\
\text { HTGR- } \\
\text { CA2.2, MSR- } \\
\text { CA2.3, FHR- } \\
\text { CA2.4 }\end{array}$ \\
\hline
\end{tabular}

\section{D.2.2 Reactor-specific radionuclides and chemical forms released}

\section{Assessing radionuclides and associated chemical forms for inclusion in advanced reactor consequence analysis:}

Radionuclide and chemical form characterization refer to the released material characterization of the source term. Current MST codes and calculations have focused on LWR technologies, but for advanced reactors these same assumptions are no longer applicable. For advanced reactors, differences in radionuclides released are possible with changes in isotopic inventory, and differences in chemical form are possible when the oxygen potential within the system is substantially different than that of an LWR, where steam is usually the dominant gas-phase component. More important, fission products may interact with coolant and other materials, e.g., sodium or molten salt, that are not present in an LWR, to form new chemical forms. Additionally, new chemical forms may behave differently when released to the environment and therefore have different downstream health effects, for example changes in water transport and resulting changes to the ingestion exposure pathway.

Currently, 69 radionuclides are typically used for LWR consequence analysis modeling in MACCS, in accordance with the NRC's State-of-the-Art Reactor Consequence Analysis Project [61]. However, the flexibility of MACCS allows for a much greater selection of 150 radionuclides, with an additional ability to expand in the future to 825 radionuclides. A study was conducted in 2020 which analyzed which additional radionuclides, and associated chemical forms, would potentially require inclusion in advanced reactor consequence analysis modeling, and provided tables of suggested radionuclides and chemical forms for inclusion in advanced reactor consequence analysis [62]. This study took into account estimates for reactor inventories and activation products, which is an area in need of further refinement and represents a prerequisite to the activity (See Section D.2.1).

Resolving this gap would include a refinement of [62] once more specific inventory data is available. This refinement would focus on additional radionuclides of interest for consequence assessments and changes to the chemical forms that might result in differences for health effects modeling, for example H-3, C-14, and Ag-110m. This item is rated as high priority and near term due to its position as a starting point for advanced reactor consequence analysis. The effort is rated as medium due to the extensive progress already made in [62]. 


\begin{tabular}{|l|c|c|c|c|}
\hline Gap Resolution Action & Priority & Effort & Timeline & $\begin{array}{c}\text { NRC Vol3 } \\
\text { Crosscut }\end{array}$ \\
\hline $\begin{array}{l}\text { Assessing radionuclides and associated chemical } \\
\text { forms needed for inclusion in advanced reactor } \\
\text { consequence analysis. }\end{array}$ & High & Med & $\begin{array}{c}\text { Near- } \\
\text { term }\end{array}$ & $\begin{array}{c}\text { SFR-CA2.1, } \\
\text { HTGR- } \\
\text { CA2.2, MSR- } \\
\text { CA2.3, FHR- } \\
\text { CA2.4 }\end{array}$ \\
\hline
\end{tabular}

\section{D.2.3 Chemical hazard modeling}

Assessing need for chemical hazard modeling needed for inclusion in advanced reactor consequence analysis:

Advanced reactor source terms may include non-radioactive chemical hazards, which are currently not included for consequence assessments. For example, and MSR source term may include beryllium and chlorine gas, both of which pose chemical health hazards. Additionally, SFRs may release sodium oxides in a fire scenario, which also present chemical health hazards. Although non-radioactive chemical hazards are not currently treated in consequence assessment codes like MACCS, the capability has been developed in the past

(CHEM_MACCS) and could be re-instituted if deemed necessary for modeling the larger breadth of health effects associated with the release of advanced reactor source terms [2].

This item was ranked as medium priority, long-term, and medium effort due to the currently undefined importance of chemical releases of advanced reactor source terms. A scoping study would be recommended as an initial step towards assessing the importance, and better defining the priority, timeline, and effort required.

\begin{tabular}{|l|c|c|c|c|}
\hline Gap Resolution Action & Priority & Effort & Timeline & $\begin{array}{c}\text { NRC Vol3 } \\
\text { Crosscut }\end{array}$ \\
\hline $\begin{array}{l}\text { Assessing need for chemical hazard modeling } \\
\text { needed for inclusion in advanced reactor } \\
\text { consequence analysis. }\end{array}$ & Med & Med & $\begin{array}{c}\text { Long- } \\
\text { term }\end{array}$ & TN-CA7 \\
\hline
\end{tabular}

\section{D.3Deposition Behavior}

\section{D.3.1 Characterize various chemical forms}

\section{Characterize unique deposition behavior for released radionuclides and associated chemical forms:}

Once more refined radionuclide and chemical form characterizations are available, additional refinement is necessary to accurately characterize atmospheric transport and dispersion as some chemical forms are reactive and/or hygroscopic, and therefore may evolve after release and alter the deposition behavior. Currently, the deposition velocity in MACCS is constant for a particular chemical group and particle size bin, which takes into account surface roughness and windspeed. However, this treatment does not take into account evolving species with the potential to change chemical forms, and therefore change deposition velocities. Furthermore, this method also does not take into account varying shape factors, and simply models all particles with a shape factor of unity representing a perfect sphere. An alternative, resistance 
model to represent deposition velocities has been proposed to account for these characteristics [39].

This item was ranked as medium priority, long-term, and medium effort due to the currently undefined importance of unique deposition behavior on advanced reactor source terms. A scoping study would be recommended as an initial step towards assessing the importance, and better defining the priority, timeline, and effort required.

\begin{tabular}{|l|c|c|c|c|}
\hline Gap Resolution Action & Priority & Effort & Timeline & $\begin{array}{c}\text { NRC Vol3 } \\
\text { Crosscut }\end{array}$ \\
\hline $\begin{array}{l}\text { Characterize unique deposition behavior for } \\
\text { released radionuclides and associated chemical } \\
\text { forms. }\end{array}$ & Med & Med & $\begin{array}{c}\text { Long- } \\
\text { term }\end{array}$ & TN-CA5 \\
\hline
\end{tabular}

\section{D.4Tritium Modeling}

\section{D.4.1 Atmospheric dispersion of tritium}

Creation of tritium specific atmospheric transport and dispersion modeling capabilities: Tritium in particular is expected to be present in MSR systems due to neutron absorption of lithium, however the transport behavior of tritium is not currently modeled in consequence analysis tools such as MACCS. A new chemical class and dispersion model would be required to characterize the atmospheric dispersion of tritium, taking into account the absorption and revaporization of tritiated water vapor on surfaces, along with associated dose conversion factors to model the resulting health effects.

This item was ranked as medium priority, long-term, and medium effort due to the currently undefined importance of tritium modeling on advanced reactor source terms. A scoping study would be recommended as an initial step towards assessing the importance, and better defining the priority, timeline, and effort required.

\begin{tabular}{|l|c|c|c|c|}
\hline Gap Resolution Action & Priority & Effort & Timeline & $\begin{array}{c}\text { NRC Vol3 } \\
\text { Crosscut }\end{array}$ \\
\hline $\begin{array}{l}\text { Creation of tritium specific atmospheric transport } \\
\text { and dispersion modeling capabilities }\end{array}$ & Med & Med & $\begin{array}{c}\text { Long- } \\
\text { term }\end{array}$ & MSR-CA4 \\
\hline
\end{tabular}

\section{D.5Dose Conversion Factors}

\section{D.5.1 Applicability of existing DCFS}

Assessing applicability of existing dose conversion factors given changes in radionuclides released, associated chemical forms, and particle size distributions:

Changes in radionuclides, chemical forms, and their associated particle sizes and deposition behavior are all important for not only characterizing concentrations, but also for accurately characterizing health effects following exposure. For example, different chemical forms can lead to solubility changes in the human body and influence the equivalent dose calculations. Dose conversion factors are responsible for translating the dose received via an exposure 
pathway into the equivalent dose to either a specific organ or effectively to the whole body. Dose conversion factors are in existence for each of the 825 radionuclides referenced in Section D.2, and are based on assumptions of releases for LWRs. However, dose conversion factors are based on a 1 micron median particle diameter and assumed dominant chemical forms, which may require re-evaluation for smaller particle sizes anticipated from non-LWRs [2] and different chemical forms, all of which have the potential to impact dosimetry and health effect calculations [39].

This item was ranked as medium priority, long-term, and medium effort due to the currently undefined importance of differences in dose conversion factors on advanced reactor source terms. A scoping study would be recommended as an initial step towards assessing the importance, and better defining the priority, timeline, and effort required.

\begin{tabular}{|l|c|c|c|c|}
\hline Gap Resolution Action & Priority & Effort & Timeline & $\begin{array}{c}\text { NRC Vol3 } \\
\text { Crosscut }\end{array}$ \\
\hline $\begin{array}{l}\text { Assessing applicability of existing dose } \\
\text { conversion factors given changes in radionuclides } \\
\text { released, associated chemical forms, and particle } \\
\text { size distributions. }\end{array}$ & Med & Med & $\begin{array}{c}\text { Long- } \\
\text { term }\end{array}$ & MSR-CA4 \\
\hline
\end{tabular}

\section{D.6Decontamination Costs}

\section{D.6.1 Applicability to advanced reactors}

\section{Assessing decontamination cost estimates given changes in radionuclides released and associated chemical forms:}

The economics of decontamination are driven by the cost effectiveness of decontamination methods compared to the interdiction or permanent condemnation of the property.

Decontamination methods are well characterized for LWRs, both in terms of effective methods to decontaminate and their associated costs. However, if the radionuclides and associated chemical forms are different, as anticipated, different decontamination methods will need to be developed along with estimates of their cost to accurately factor into economic consequence modeling. For example, tritium permeates easily through metals, creating a challenge for decontamination methods. Furthermore, decontamination estimates for consequence analysis tools such as MACCS are currently based on rural and suburban environments. With the expectation that non-LWRs may be closer to urban environments, more complex structures and materials may need to be considered in refining decontamination models [2].

This item was ranked as medium priority, long-term, and medium effort due to the currently undefined importance of decontamination methods and costs on advanced reactor source terms. A scoping study would be recommended as an initial step towards assessing the importance, and better defining the priority, timeline, and effort required.

\begin{tabular}{|l|c|c|c|c|}
\hline Gap Resolution Action & Priority & Effort & Timeline & $\begin{array}{c}\text { NRC Vol3 } \\
\text { Crosscut }\end{array}$ \\
\hline $\begin{array}{l}\text { Assessing decontamination cost estimates given } \\
\text { changes in radionuclides released and associated } \\
\text { chemical forms. }\end{array}$ & Med & Med & $\begin{array}{c}\text { Long- } \\
\text { term }\end{array}$ & TN-CA6 \\
\hline
\end{tabular}




\section{D.7Multi-Unit Consequence Assessment}

\section{D.7.1 Consequence modeling of multi-unit sites}

Evaluate simplification methods for conducting multi-unit consequence assessments: Several advanced reactor designs propose two or more reactors per site, thus the potential need for modeling many combinations of source terms at a time represents a current gap. The number of required consequence analyses to treat all the possible combinations is determined by the number of units $(\mathrm{M})$, the number of source term categories $(\mathrm{N})$, and by the formula: $(\mathrm{N}+\mathrm{M}) ! /[\mathrm{N} ! \times \mathrm{M} !]-1$, which can become rather unrealistic for even modest values of $\mathrm{M}$ and $\mathrm{N}$ [63]. Therefore, a simplified approach to performing multi-unit consequence analysis will be needed in order to meet licensing requirements and appropriately size the EPZ [63]. This simplified approach could consist of methods to group source terms into categories to minimize the required calculations. Utilizing these simplification methods, the number of analyses needed is reduced by several orders of magnitude and are far more accessible computing wise even for high numbers of reactor units and source terms [63].

This item was ranked as medium priority, long-term, and medium effort due to the currently undefined importance of multi-unit consequence assessments on reactor source terms. A scoping study would be recommended as an initial step towards assessing the importance, and better defining the priority, timeline, and effort required.

\begin{tabular}{|l|c|c|c|c|}
\hline Gap Resolution Action & Priority & Effort & Timeline & $\begin{array}{c}\text { NRC Vol3 } \\
\text { Crosscut }\end{array}$ \\
\hline $\begin{array}{l}\text { Evaluate simplification methods for conducting } \\
\text { multi-unit consequence assessments. }\end{array}$ & Med & Med & $\begin{array}{c}\text { Long- } \\
\text { term }\end{array}$ & \\
\hline
\end{tabular}


Argonne

\section{Nuclear Science and Engineering}

Argonne National Laboratory

9700 South Cass Avenue, Bldg. 208

Argonne, IL 60439

www.anl.gov

Argonne National Laboratory is a U.S. Department of Energy laboratory managed by UChicago Argonne, LLC 